\title{
RECENT DEVELOPMENTS IN DYNAMICAL SYSTEMS: THREE PERSPECTIVES
}

\author{
F. BALIBREA \\ Universidad de Murcia, Dpto. de Matemáticas, \\ 30100-Murcia, Spain \\ E-mail: balibrea@um.es \\ T. CARABALLO \\ Universidad de Sevilla, Dpto. Ecuaciones Diferenciales y Análisis \\ Numérico, \\ Apdo. de Correos 1160, 41080-Sevilla (Spain) \\ E-mail: caraball@us.es \\ P.E. KLOEDEN \\ Institut für Mathematik, Goethe-Universität, \\ D-60054 Frankfurt am Main, Germany \\ E-mail: kloeden@math.uni-frankfurt.de

\section{J. VALERO} \\ Univ. Miguel Hernández de Elche, Centro de Investigación Operativa, \\ Avda. Universidad s/n, 03202 Elche (Alicante), Spain \\ E-mail: jvalero@umh.es
}

Received September 15, 2009; Revised October 30, 2009

Dedicated to the memory of Valery S. Melnik

\begin{abstract}
The aim of this paper is to give an account of some problems considered in past years in the setting of Dynamical Systems, some new research directions and also state some open problems
\end{abstract}

Keywords: Topological entropy, Li-Yorke chaos, Lyapunov exponent, continua, nonautonomous systems, difference equations, ordinary and partial differential equations, set-valued dynamical system, global attractor, non-autonomous and random pullback attractors.

Mathematics Subject Classifications (2000): 35B40, 35B41, 35K40, 35K55, 37B25, 37B30, 37B40, 37B45, 37B55, 37E05, 37E15, 39A20 58C06, 60H15.

\section{Introduction}

The theory of dynamical systems has a long prehistory, but essentially started in its present form with the work of Poincaré and Birkhoff on problems con- nected with celestial mechanics. In particular, the theory of discrete dynamical systems, which mainly uses iteration theory, is one of the most relevant topics in the subject.

The purpose of this tutorial is to give a par- 
tial account of the progress obtained in discrete and continuous systems is recent years, to present some open and new problems. It consists of four sections. In Sections 2 and 3 we consider some results in the topological dynamics in non-autonomous discrete systems and in Section 4 we give a rather complete review of multi-valued dynamical systems arising from models involving partial differential equations. Finally, in Section 5 we provide an overview of many problems in non-autonomous and random dynamical systems, in particular on new concepts of nonautonomous and random attractors.

\section{Autonomous discrete dynamical systems}

The development of the theory of topological dynamics began in the earlier part of the last century. It focused in particular on problems related to autonomous discrete dynamical systems given by the pair $(X, f)$, where $X$ is a topological space and $f$ a continuous map of $X$ into itself. The crucial problem was the study of properties of all orbits of all points in the space state $X$. For $x \in X$, the orbit of $x$ by $f$ is the sequence $\left(f^{n}(x)\right)_{n=0}^{\infty}$, where $f^{n}=$ $f\left(f^{n-1}\right)$ for all $n \geq 1$ and $f^{0}=i d_{X}$ (identity on $X$ ). In most cases $X$ is a compact metric space and, in particular, extensive results were obtained when $X$ $=I=[a, b]$ because many phenomena from social, natural and economical sciences can be formulated as systems evolving with time in a discrete way in such spaces. Moreover, when $X=\mathbb{R}^{d}$, or a subset thereof, we generally speak of problems on difference equations.

Although the number of new results has been impressive, we will include here some of them from one of the subjects more active in the field in last years, dynamical systems on continua.

\subsection{Autonomous dynamical systems on continua}

One line of research that has been very active in recent years is that of dynamical systems on continua, i.e., where $X$ is a continuum (a compact and connected topological space) and $f \in C(X, X)$. For definitions and detailed account of results see [Nadler, 1995]. Problems such as periodic structure using methods from combinatorial dynamics have been studied on circles, trees and finite graphs (see [Alsedà et al., 2000]), as well as on centers and depth of centers [Ye, 1993, Kato, 1995, Kato, 1998, Efremova \& Makhrova, 2003] on trees, graphs and dendrites, and on the structure of $\omega$-limit sets [Kocan et al., 2010, Balibrea \& García Guirao, 2005], in particular, $\omega$-limit sets on hereditarily locally connected continua [Spitalský, 2008], etc.

Within dynamical systems theory one of the most interesting topics is that of minimal systems, i.e., systems that do not contain non-trivial subsystems. A system $(X, f)$ is minimal if there is no proper subset $Y \subseteq X$ which is non-empty, closed and $f$-invariant, i.e. satisfies $f(Y) \subseteq Y$. It is immediate that $(X, f)$ is minimal if and only if the forward orbit of all points in $X$ are dense in $X$. We will say also that in this case $f$ is also minimal. Here we will concentrate on the progress on minimal systems in the case that the phase space $X$ is one-dimensional.

The topological characterization of minimal sets of one-dimensional $X$ has been carried out for intervals, circles, trees, finite graphs and dendrites. In the interval case, these are finite and Cantor sets (see [Block \& Coppel, 1992]), while for the circle case the circle itself can also be minimal. These results can be generalized to graphs, where minimal sets are characterized as finite sets, Cantor sets and also unions of finitely many pairwise disjoint circles [Balibrea et al., 2003, Mai, 2005].

A dendrite is defined as a locally connected continuum which contains no simple closed curve. In this case, besides partial results in [Balibrea et al., 2003], a complete characterization has been given recently in [Balibrea et al., 2009] as a consequence of a more general result based on the new notion of almost totally disconnected spaces. A space $X$ is almost totally disconnected if the set of its degenerate components is dense in $X$. The main results says that an almost totally disconnected space admits a minimal map if and only if it is either a finite set or has no isolated point. By a brain we mean a cantoroid whose degenerate components are dendrites and form a null family (for any $\varepsilon>0$, only a finite number of its members have diameters greater than $\varepsilon$ ). A cantoroid is a compact metric and almost totally disconnected space without isolated points. With these ingredients we can now state the characterization in [Balibrea et al., 2009]. 
Theorem 2.1. Let $D$ be a dendrite and let $M$ be a subset of $D$. Then $M$ is a minimal set for some dynamical system $(D, f)$ if and only if $M$ is either a finite set or a brain.

In addition, the following characterization was given in [Balibrea et al., 2009] for general almost totally disconnected spaces.

Theorem 2.2. An almost totally disconnected compact metric space admits a minimal dynamical system if and only if it is either a finite set or a cantoroid.

Question 2.3. Is it possible to give a topological characterization of minimal sets in other families of one-dimensional continua like arc-like, tree-like or circle-like? (For definitions see [Nadler, 1995]).

\section{Non-autonomous discrete systems}

We now consider the situation in which the map describing the evolution of the dynamics is itself allowed to change with time. This admits the following formulation. Given a compact topological space $X$ and a sequence of continuous self-maps $\left(f_{n}\right)_{n=1}^{\infty}$ $=f_{1, \infty}$ from $X$ into itself, the pair $\left(X, f_{1, \infty}\right)$ will be called a non-autonomous discrete system where the orbit of a point $x \in X$ is described by the sequence

$$
\left.x, f_{1}(x), f_{2}\left(f_{1}(x)\right), \ldots, f_{n}\left(f_{n-1}\right) \ldots\left(f_{2}\left(f_{1}(x)\right)\right) \ldots\right)
$$

We will use the notation

$$
f_{1}^{n}=f_{n} \circ f_{n-1} \circ \ldots \circ f_{2} \circ f_{1}
$$

which was introduced in [Kolyada \& Snoha, 1996].

When all of the maps are the same, i.e., $f_{n}=f$ for all $n \in \mathbb{N}$, then we have a autonomous discrete dynamical system or, simply, a discrete dynamical system, which we considered above.

There is not a large literature on truly nonautonomous discrete systems and, as consequence, there are a few results. In the following subsections we will survey some interesting developments. Other results can be found in the expository article [Kloeden, 2000].

\subsection{Topological entropy}

The authors of [Kolyada \& Snoha, 1996] defined the topological entropy of a non-autonomous dis- crete system, which they denoted by $h\left(f_{1, \infty}\right)$, using the technique of open covers of $X$ as in the original paper [Adler et al., 1965] on autonomous systems. In the case that $X$ is metric or metrizable, they also used separated and spanning sets as in [Bowen, 1970]. It is easy to see that such definitions give similar results when the system is in fact autonomous. What is really interesting, is that it is now possible to define the entropy $h\left(f_{1, \infty}, Y\right)$, when $Y$ is a subset of $X$ which is not necessarily compact or invariant under $f\left(f_{1, \infty}\right)$. Such an extension was necessary to deal with other problems considered in [Kolyada \& Snoha, 1996].

An interesting and surprising consequence of the paper [Kolyada \& Snoha, 1996] was a proof of the commutativity of the entropy autonomous dynamical systems, i.e., the entropy of the composition of two continuous self-maps on a compact space does not depend on the order in which they are taken, i.e., $h(f \circ g)=h(g \circ f)$. The first time that the commutativity of the entropy was mentioned seems to have been in [Dinaburg, 1970].

Inspired by [Kolyada \& Snoha, 1996], the commutativity or non-commutativity of other types of entropy such sequence entropy were proved in [Balibrea et al., 1999a, Balibrea et al., 1999b]).

It is well known (see [Misiurewicz,1989]) that for interval maps, the entropy is positive if and only if one of its iterates has a structure called horseshoe. An interval map $f$ has a horseshoe if there are two disjoint intervals $J$ and $K$ such that $f(J) \cap f(K) \supset J \cup K$. It is difficult to extend this definition to non-autonomous interval systems because it is associated to a unique map and $f_{1, \infty}$ is a sequence. If such notion were possible while retaining properties similar to those of horseshoes for autonomous dynamical interval systems, then one can ask:

Question 3.1. If a non-autonomous interval system $f_{1, \infty}$ has positive entropy, does it possess a structure of horseshoe type?

Another way to obtain non-autonomous dynamical systems is to allow not only maps to change, but also spaces. This leads to the following generalization: a non-autonomous dynamical system is the pair $(X, f)$ given by $X=\left(X_{n}\right)_{n=1}^{\infty}$ and $\left.f=\left(f_{n}\right)_{n=1}^{\infty}\right)$, where each $X_{n}$ is a compact metric 
space and $f_{n}: X_{n} \rightarrow X_{n+1}$ for $n \in \mathbb{N}$. Such a notion is used for example in [Kolyada et al., 1999] to construct a class of smooth triangular maps on the unit square of type $2^{\infty}$ having positive topological entropy and thus extending a previous result from [Balibrea et al., 1995]. Another example is to consider skew-product maps which were introduced in [Bowen, 1970] and are often called triangular maps when defined on $[0,1]^{2}$, i.e. with

$$
F(x, y)=(f(x), g(x, y))=\left(f(x), g_{x}(y)\right),
$$

where $f \in C(I, I)$ and $g_{x} \in C(I, I)$ are continuous. Dynamical systems generated by continuous maps on hereditarily locally connected continua such as dendrites can also be interpreted as a sequence of spaces and maps between them and hence as as generalized non-autonomous systems. The sequence of spaces can be, for example, trees approaching dendrites (see [Nadler, 1995]). Results on entropy can be also obtained in such cases.

The above generalization was used also to obtain a formula for entropy in terms of the number of pieces of monotonicity of the functions $f_{1}^{n}$ when all the $X_{n}$ are compact real intervals and maps are piecewise monotone. This is a generalization of the well-known Misiurewicz-Szlenk formula for a continuous piecewise interval map (see [Misiurewicz \& Slenk,1980]).

\subsection{Periodic non-autonomous systems}

We will restrict now our attention to the case $X_{n}=$ $I$ or $\mathbb{R}$ for each $n \in \mathbb{N}$ since a lot of results have been obtained in this setting. Let us suppose that there exists a positive integer $p$ (prime period) such that $f_{n+p}=f_{n}$ for each $n \geq 0$. Then the system

$$
x_{n+1}=f_{n}\left(x_{n}\right)
$$

is called a p-periodic non-autonomous system.

In the setting of economical theory, the two periodic case has an interpretation in terms of Parrondo's paradox [Cánovas et al., 2006]. This paradox says that two losing games can result, under random or periodic alternation of their dynamics, in a winning game. That is, it can happen that combination of losing + losing can result winning. For more information on this paradox see [Harmer \& Abbott, 1999a, Harmer \& Abbott, 1999b,
Parrondo, Harmer \& Abbott, 2000]. A partial analysis of when the paradox is impossible is given in [Cánovas, 2010]. It would be interesting to consider examples in two dimensional settings and analyse whether the paradox is possible or not.

Motivated by Sharkovskii's results on the coexistence of periodic orbits of certain periods ([Sharkovskii, 1994, Sharkovskii, 1964]) and forcing relationships, we wonder if the same type of such relationships can be obtained in the non-autonomous case. There are several papers on this for interval maps ([Alsharawi et al., 2006, Alves, 2009, Cánovas \& Linero, 2006]). It is stated in [Alves, 2009] that if the condition

$$
\operatorname{Card}\left\{x \in I, f_{i}(x)=f_{j}(x)\right\}<\infty, \forall i \neq j(\bmod p)
$$

holds, then if the system has a periodic orbit of period $r$ such that $\frac{\operatorname{lcm}(r, p)}{p}$ is odd and larger than 1, then the system has periodic orbits of all periods forced by the Sharkovskii ordering. When $p=1$ we recover the Sharkovskii's original result. To clarify the situation, the sets

$$
\mathfrak{A}_{q}=\left\{n \in \mathbb{Z}^{+}: \operatorname{lcm}(n, p)=p q\right\} \text { for } q \in \mathbb{Z}^{+}
$$

were introduced in [Alsharawi et al., 2006]. If $\prec$ denotes Sharkovskii's ordering, then we have

Theorem 3.2. If $\mathfrak{A}_{l} \cap \mathfrak{P} \neq \emptyset$ for some $l \in \mathbb{Z}^{+}$, then $\mathfrak{A}_{q} \cap \mathfrak{P} \neq \emptyset$ for all $l \prec q$.

It is necessary distinguish the periods of the $p$ periodic equation in $\mathfrak{A}_{q}$. To this end we introduce

$$
\mathfrak{Q}=\{n \in \mathfrak{P}: p \nmid n\} .
$$

Note, by the definition of $\mathfrak{A}_{q}$, we have $\mathfrak{A}_{q} \cap p \mathbb{Z}^{+}=$ $\{p q\}$ for every $q \in \mathbb{Z}^{+}$. As a consequence $\mathfrak{A}_{q} \cap \mathfrak{P}=$ $\{p q\}$ whenever $\mathfrak{A}_{q} \cap \mathfrak{P} \neq \emptyset$ and $\mathfrak{A}_{q} \cap \mathfrak{Q}=\emptyset$. Finally, if $l \in \mathbb{Z}^{+}$, then

$$
\mathfrak{S}_{l}=\left\{p q \in \mathbb{Z}^{+}: q \prec l, \text { or } \mathfrak{A}_{q} \cap \mathfrak{Q} \neq \emptyset\right.
$$

With such notation we reformulate the former theorem.

Theorem 3.3. If $\mathfrak{A}_{l} \cap \mathfrak{P} \neq \emptyset$ for some $l \in \mathbb{Z}^{+}$, then $\mathfrak{S} \backslash \mathfrak{S}_{l}=\left(\mathfrak{Q} \cup p \mathbb{Z}^{+}\right) \backslash \mathfrak{S}_{l}$

Reasons for interest of knowing the elements of $\mathfrak{Q}$ are given in [Alves, 2009] and the 
case of two periodic equations is studied in [Cánovas \& Linero, 2006], where it is shown that if such an equation has an odd period larger than 1 , then

$$
\mathfrak{P}=\mathfrak{Q} \cup 2 \mathbb{Z}^{+} .
$$

Such results underline the role played by the set $\mathfrak{Q}$.

Question 3.4. Is it possible to obtain a general formula for all periods of a p-periodic difference equation similar to that above?

\subsection{Lyapunov exponents in periodic non- autonomous systems}

Let us start with an example coming from using the Poincaré map to understand the behavior of orbits in non-linear and non-autonomous differential equations of second order (see for example [Chacón, 2001]), namely

$$
x_{n+1}=\left[\alpha+\varepsilon\left(b_{n}+\beta c_{n}\right)\right] x_{n}=a_{n} x_{n}
$$

with $\alpha>1,0<\beta<1, b_{n}=\sqrt{2} \sin n, c_{n}=$ $\sqrt{2}[2 K(m)(n+\Theta) / \pi ; m]$. That is, for the sake of clarity and possibility of comparing results we choose the main resonance regime $T_{\sin }=T_{s n}=2 \pi$, where $s n$ denotes the Jacobian elliptic function of parameter $m$, while $K(m)$ is the complete elliptic integral of the first kin and $\Theta$ is the initial phase, i.e. with $0 \leq \Theta \leq 2 \pi$.

The model is a particular case of general nonautonomous systems $x_{n+1}=f_{n}\left(x_{n}\right)$, where $f_{1}, f_{2}$, ... are continuous maps of $X$ into itself, and $X=$ $\mathbb{R}$ or $X=I=[0,1]$. The form of the perturbation is designed to capture only the effect of weak non-autonomous excitations on the stability of a generic unstable limit cycle. The elliptic function $s n$ is chosen to introduce in the control excitation in a simple way the effect of the excitation waveform on the control scenario.

The behavior of the system is investigated by exploiting the connection between the sensitivity to initial conditions and Lyapunov exponents in autonomous systems: when a point $x \in X$ has a positive Lyapunov exponent, then its orbit and that of a point nearby diverge at a positive exponential rate. This connection was explored in [Abraham et al., 2004], while the connection between Lyapunov exponents and positive metric entropy was clarified in [Barrio, 2007].
We can extend the notion of Lyapunov exponent for an autonomous dynamical system $(X, f)$ to a non-autonomous systems $\left(X, f_{1, \infty}\right)$ by the formula

$$
\begin{aligned}
\lambda(x) & =\lim _{n \rightarrow \infty} \frac{1}{n} \log \left|\left(f_{n} \circ \ldots f_{2} \circ f_{1}\right)^{\prime}(x)\right| \\
& =\lim _{n \rightarrow \infty} \frac{1}{n} \sum_{j=1}^{n-1} \log \left|f_{j}^{\prime}(x(j))\right|
\end{aligned}
$$

As a consequence we can give a notion of chaotic behavior for systems. We will say that a nonautonomous discrete system has chaotic behavior if there is a Lebesgue measurable set of points $L$ in the space state $X$ with positive Lyapunov exponent (in the extended sense). In turn this is interpreted in the application considered in the above paper as the existence of homoclinic chaos, i.e., the existence in the phase space of the non-linear second order differential equation of a homoclinic orbit in the neighborhood of a separatrix. The system is non-chaotic if there is no such set $L$.

Exercise 3.5. Propose new examples and applications of the extension of Lyapunov exponents and develop a complete theory in the setting of $\left(X, f_{1, \infty}\right)$ with $X=I$ or $X=\mathbb{R}$.

\subsection{Li-Yorke chaos in non-autonomous systems}

Since the notion of chaos in the sense of $\mathrm{Li}$ and Yorke is given in terms of the behavior of orbits of points in $X$, we can extend it to the setting of nonautonomous systems given by sequences of maps $f_{1, \infty}$

Definition 3.6. The system $\left(X, f_{1, \infty}\right)$ is chaotic in the sense of $\mathrm{Li}$ and Yorke (resp., $\delta$-chaotic) if there is an uncountable set $S \subset X$ (called a scrambled set) such that for all pairs $x, y \in X$ with $x \neq y$

$$
\begin{aligned}
& \limsup _{n \rightarrow \infty} d\left(f_{1}^{n}(x), f_{1}^{n}(y)\right)>0(>\delta, \text { resp. }) \\
& \liminf _{n \rightarrow \infty} d\left(f_{1}^{n}(x), f_{1}^{n}(y)\right)=0 .
\end{aligned}
$$

As a consequence, we can state similar problems in this new setting similar to what has been proved in the case of autonomous discrete dynamical systems. 
Exercise 3.7. Prove or disprove that, when $X=$ $I$, if there is a Li-Yorke pair, then there is an uncountable number of pairs.

For topological entropy we have

Question 3.8. If $h\left(f_{1, \infty}\right)>0$, is it true that $f_{1, \infty}$ is Li-Yorke chaotic?

There are many problem for autonomous systems with zero topological entropy which can also be considered in the setting of non-autonomous systems.

\subsection{Li-Yorke chaos in $\mathbb{R}^{n}$ with $n>1$}

The notion of snap-back repeller for maps $f \in$ $C^{1}\left(\mathbb{R}^{n}, \mathbb{R}^{n}\right)$ was introduced in [Marotto, 1978] as follows. Assume that $x^{\star}$ is an expanding fixed point of $f$ in the ball $B_{r}\left(x^{\star}\right)$, i.e. where $f\left(x^{\star}\right)=x^{\star}$, and all eigenvalues of $D f(x)$ are greater than one in norm for every $\left.x \in B_{r}\left(x^{\star}\right)\right)$. Then $x^{\star}$ is a snapback repeller for $f$ if there is a point $x_{0} \in B_{r}\left(x^{\star}\right)$ with $x_{0} \neq x^{\star}$ such that $f^{p}\left(x_{0}\right)=x^{\star}$ for some positive integer $p$ and the determinant $\operatorname{det} D f^{p}\left(x_{0} \neq\right.$ 0 .

In the same paper, Marotto proved that the existence of a snap-back repeller is a sufficient condition to have Li-Yorke chaos (in a generalized sense of the usual notion). Marotto's theorem was improved after by a theorem of Shi and Chen in [Shi \& Chen, 2004]. Other extensions to the Banach space setting can be found in [Kloeden \& Li, 2006] .

Exercise 3.9. It would be interesting to know if such theory could be extended to non-autonomous systems. That would require also extending the notion of snap-back repeller to another notion available in the new setting.

\subsection{Non-autonomous difference equations}

The field of difference equations has developed quickly over the last thirty years. In many cases, problems can be formulated in terms of autonomous systems given by the pair $(X, f)$ where $X$ is either $(0, \infty)$ or $\mathbb{R}$ and $f: X \rightarrow X$ is a non necessarily continuous map. The main problem is the study of properties of solutions of the equation which are given by the sequences $\left(x_{n}\right)_{n=0}^{\infty}$ where $x_{0}$ is the initial point and $x_{n}=f^{n}\left(x_{0}\right)$ with the same meaning that in previous paragraphs.

In many recent applications we have to consider $x_{n+1}=f_{n}\left(x_{n}\right)$, where $\left(f_{n}\right)_{n=0}^{\infty}$ is a sequence of maps of $X$ into itself, which need not be continuous. The traditional problems to be solved are: boundedness, periodicity, convergence, local and global stability of solutions. Equations with delays which are also important in applications are formulated by

$$
x_{n+1}=f_{n}\left(x_{n}, x_{n-1}, \ldots, x_{n-k}\right),
$$

where $f_{n}: \mathbb{R}^{k+1} \rightarrow \mathbb{R}$ is a continuous map for each $n=0,1, \ldots, k \geq 1$ and $x_{-k}, x_{-k+1}, \ldots, x_{0} \in$ $\mathbb{R}^{1}$. These can be reformulated as first order (i.e. without delay) vector valued difference equations in terms of $\mathbb{X}_{n}:=\left(x_{n-k}, x_{n-k+1}, \ldots, x_{n}\right) \in \mathbb{R}^{k+1}$.

\section{6(A). Examples of non-autonomous rational equations}

Non-autonomous rational equations are nonautonomous differential equations involving rational functions. They are common in applications in population dynamics (see [Kulenovic \& Ladas, 2002]).

1. The non-autonomous delay Pielou logistic equation:

$$
x_{n+1}=\frac{a_{n} x_{n}}{1+x_{n-k}},
$$

where $\left(a_{n}\right)_{n=0}^{\infty}$ is a positive periodic sequence with period $p$, i.e. with

$$
a_{n+p}=a_{n}, \quad n=0,1, \ldots
$$

When $a_{n}=\alpha$ the equation is autonomous and the asymptotic behavior and oscillation of its positive solutions have been studied in [Kulenovic \& Ladas, 2002].

When $\left(a_{n}\right)_{n=0}^{\infty}$ is bounded and persistent (below bounded), [Kocic \& Ladas, 1993] gives sufficient conditions for boundedness and the global attractivity of solutions. In the periodic case, sufficient conditions in the coefficients which ensure that there are periodic solutions are also given. 
2. The previous model is an extension of the periodically forced Beverton-Holt model

$$
x_{n+1}=\frac{r K_{n} x_{n}}{K_{n}+(r-1) x_{n}},
$$

which represents a population with inherent growth rate $r>1$ and a carrying capacity represented by the periodic positive sequence $\left(K_{n}\right)_{n=1}^{\infty}$ which is assumed to have a prime period $p \geq 2$.

3. Non-autonomous Lyness equation: Let $\left(b_{n}\right)_{n=1}^{\infty}$ be a sequence of positive numbers. The non-autonomous Lyness equation

$$
x_{n+1}=\frac{x_{n}+b_{n}}{x_{n-1}}, \quad x_{0}>0, x_{1}>0,
$$

is a non-autonomous version of the Lyness equation given by

$$
x_{n+1}=\frac{x_{n}+b}{x_{n-1}}, \quad x_{0}>0, x_{1}>0,
$$

which is well-known in the literature. See for example [Camouzis \& Ladas, 2007], where a complete report of such equation is given and stated some interesting open problems. In the non-autonomous case, it has been conjectured that solutions are bounded and persist (i.e. are bounded away form zero and infinity) if $\left(b_{n}\right)_{n=1}^{\infty}$ has the same properties. One can ask which other more general sequences $\left(b_{n}\right)_{n=1}^{\infty}$ ensure that the solution $\left(x_{n}\right)_{n=0}^{\infty}$ is bounded and persistent. An interesting example in [Angelis, 2004] shows that $\left(x_{n}\right)_{n=0}^{\infty}$ can even be non-bounded when $\left(b_{n}\right)_{n=1}^{\infty}$ attains only two values and proves that is sufficient for $\left(b_{n}\right)_{n=1}^{\infty}$ to be a monotone sequence to prove that the conjecture is true.

4. It is well-known [Camouzis \& Ladas, 2007] that the solutions of the difference rational equation with two delays

$$
x_{n+1}=\frac{x_{n}+x_{n-2}}{x_{n-1}},
$$

tends to a period-four solution. It was shown for extension of the model in [Papaschinopoulos \& Schinas, 2008],

$$
\begin{gathered}
x_{n+1}=\frac{p_{n}+q_{n} x_{n}+q_{n}}{x_{n-k}}, \\
n=0,1, \ldots, k=1,2, \ldots,
\end{gathered}
$$

with positive initial values $x_{-k}, x_{-k+1}, \ldots$, $x_{0}$, that the solutions are bounded, persist and have certain periodicity properties under some conditions on the sequences $\left(p_{n}\right)_{n=1}^{\infty}$ and $\left(q_{n}\right)_{n=1}^{\infty}$,

The following open problem was stated in [Papaschinopoulos \& Schinas, 2008].

Question 3.10. Consider the difference equation (3) with $k=3,4, \ldots$ where $\left(p_{n}\right)_{n=1}^{\infty}$ and $\left(q_{n}\right)_{n=1}^{\infty}$ are positive sequences such that $\left(p_{n}\right)_{n=1}^{\infty}$ is bounded and either

$$
p_{n}-p_{n-k} \geq 0 \text { and } q_{n}=q_{n-k}, \quad n=k, k+1, \ldots
$$

or

$$
p_{n}-p_{n-k} \leq 0 \text { and } q_{n}=q_{n-k}, \quad n=k, k+1, \ldots
$$

Is every solution of (3) bounded and persistent?

\section{Multi-valued dynamical systems}

\subsection{Equations without uniqueness}

The theory of semigroups of operators has been a powerful tool for studying the properties (and, in particular, the asymptotic behavior) of solutions of autonomous differential equations in partial derivatives with uniqueness of the Cauchy problem. In this situation the existence and properties of global attractors have been established for a wide type of equations such as reaction-diffusion systems, the two-dimensional Navier-Stokes equations, wave equations and many others (see, e.g., [Babin \& Vishik, 1992, Hale, 1988, Ladyzhenskaya, 1991, Sell \& You, 1995, Temam, 1988]).

However, in many situations concerning systems of physical relevance either uniqueness fails or it is not known to hold. In such cases we cannot define a classical semigroup of operators, so that another theory involving multi-valued maps is necessary, namely, the theory of multi-valued dynamical systems. Often a system is really multi-valued (example of this cases will be given) but, in other cases, we are simple not able to prove the uniqueness of solutions. Hence, the theory of multi-valued dynamical systems allows us to continue when the proof of uniqueness fails due to technical problems, 
and it is still possible to study the asymptotic behavior of solutions no matter we have uniqueness or not.

There exists now in the literature a great number of equations of physical interest for which uniqueness of solutions fails. Among these we can cite, for example, the three-dimensional NavierStokes system, the Ginzburg-Landau equation, the Lotka-Volterra system with diffusion, the wave equation or differential inclusions (including some models from climatology). This is, of course, one important reason justifying the interest of multivalued dynamical systems. Another (not less important) reason is the fact that there is usually a gap between the conditions that we need to impose to obtain existence of solutions and the conditions necessary to prove uniqueness. Hence, we can weaken the conditions imposed on a differential equations and then consider more general situations.

We shall give now in more detail some examples of equations in which either uniqueness is not true or is not known to hold.

\section{1(A). Three-dimensional Navier-Stokes equa- tions}

Let $\Omega \subset \mathbb{R}^{3}$ be a bounded open subset with smooth boundary. For given $\nu>0$ we consider the NavierStokes system

$$
\left\{\begin{array}{l}
\frac{\partial u}{\partial t}-\nu \Delta u+(u \cdot \nabla) u=-\nabla p+f, \text { in }(0, T) \times \Omega, \\
\operatorname{div} u=0, \\
\left.u\right|_{\partial \Omega}=0, \quad u(0, x)=u_{0}(x),
\end{array}\right.
$$

where $u(t, x)=\left(u_{1}(t, x), u_{2}(t, x), u_{3}(t, x)\right)$ is the velocity of an incompressible fluid, $p$ is the pressure and $f$ is an external force.

If we consider the usual function spaces

$$
\begin{aligned}
& \mathcal{V}=\left\{u \in\left(C_{0}^{\infty}(\Omega)\right)^{3}: \operatorname{div} u=0\right\}, \\
& H=c l_{\left(L^{2}(\Omega)\right)^{3}} \mathcal{V}, V=\operatorname{cl}_{\left(H^{1}(\Omega)\right)^{3}} \mathcal{V},
\end{aligned}
$$

and assume that $f \in H$, then the following results are well known (see e.g. [Lions, 1969, Temam, 1979, Temam, 1988]):

1. For every $u_{0} \in V$ there exists a unique strong solution of problem (4) which exists in some interval $\left[0, T\left(\left\|u_{0}\right\|_{V}\right)\right)$.
2. For every $u_{0} \in H$ there exists at least one weak solution of (4) which exists in the whole half-line $[0,+\infty)$.

Therefore, strong solutions are unique, but they cannot be defined globally in time. On the other hand, weak solutions exist globally in time, but it is not known whether uniqueness holds or not.

Thus, if we intend to study the asymptotic behavior of solutions as time goes to infinity, then we need to work with weak solutions and we have a problem for which uniqueness fails.

In the two-dimensional case, i.e., $\Omega \subset \mathbb{R}^{2}$, uniqueness of weak solutions is true (see, e.g., [Temam, 1979]), so a semigroup of operators can be defined in the phase space $H$, and the existence of a finite-dimensional global attractor is a well known result (see [Ladyzhenskaya, 1982, Temam, 1988, Babin \& Vishik, 1992]).

\section{1(B). Reaction-diffusion systems}

Let $d>0$ and $N \geq 1$ be integers and let $\Omega \subset \mathbb{R}^{N}$ be a bounded open subset with smooth boundary. We denote by $|\cdot|$ the norm in the space $\mathbb{R}^{d}$ (or $\mathbb{R}$ ), and by $(\cdot, \cdot)$ the scalar product in $\mathbb{R}^{d}$. Consider the problem

$$
\left\{\begin{array}{l}
\frac{\partial u}{\partial t}-a \Delta u+f(u)=h(x), \quad(t, x) \in(0, T) \times \Omega \\
\left.u\right|_{x \in \partial \Omega}=0 \quad\left(\text { or }\left.\frac{\partial u}{\partial \nu}\right|_{x \in \partial \Omega}=0\right) \\
u(0, x)=u_{0}(x),
\end{array}\right.
$$

where $T>0, x \in \Omega, \nu$ is the unit outward normal, $u=\left(u^{1}(t, x), \ldots, u^{d}(t, x)\right), a$ is a real $d \times d$ matrix with a positive symmetric part $\frac{1}{2}\left(a+a^{t}\right) \geq$ $\beta I$ with $\beta>0$ and $h \in\left(L^{2}(\Omega)\right)^{d}$. Moreover, $f=$ $\left(f^{1}, \ldots, f^{d}\right)$ is a continuous function satisfying the following conditions:

$$
\begin{gathered}
\sum_{i=1}^{d}\left|f^{i}(u)\right|^{\frac{p_{i}}{p_{i}-1}} \leq C_{1}\left(1+\sum_{i=1}^{d}\left|u^{i}\right|^{p_{i}}\right), \\
(f(u), u) \geq \alpha \sum_{i=1}^{d}\left|u^{i}\right|^{p_{i}}-C_{2},
\end{gathered}
$$

where $p_{i} \geq 2, \alpha, C_{1}, C_{2}>0$.

Let $H=\left(L^{2}(\Omega)\right)^{d}, V=\left(H_{0}^{1}(\Omega)\right)^{d}$ for Dirichlet boundary conditions and $V=\left(H^{1}(\Omega)\right)^{d}$ for Neumann boundary conditions. Also, for $p=\left(p_{1}, \ldots, p_{d}\right)$ 
define the spaces

$$
\begin{gathered}
L^{p}(\Omega) \stackrel{\text { def }}{=} L^{p_{1}}(\Omega) \times \cdots \times L^{p_{d}}(\Omega), \\
L^{p}\left(0, T ; L^{p}(\Omega)\right) \stackrel{\stackrel{\text { def }}{=}}{=} L^{p_{1}}\left(0, T ; L^{p_{1}}(\Omega)\right) \times \\
\times \cdots \times L^{p_{d}}\left(0, T ; L^{p_{d}}(\Omega)\right) .
\end{gathered}
$$

By a globally defined weak solution of (6) we mean a function $u(\cdot)$ which belongs to $L^{\infty}(0, T ; H) \cap L^{2}(0, T ; V) \cap L^{p}\left(0, T ; L^{p}(\Omega)\right)$ for all $T>0$ and satisfies the equation in the sense of distributions. As the regularity of $u$ implies that $u \in C([0, \infty), H)$, the initial condition makes sense [Chepyzhov \& Vishik, 1996]. Under these conditions there exists at least one globally defined weak solution for every initial condition $u_{0} \in\left(L^{2}(\Omega)\right)^{d}$ [Chepyzhov \& Vishik, 2002b; p.283].

Uniqueness is true, for example, if the following conditions holds (see e.g. [Chepyzhov \& Vishik, 2002b; p.283]): the function $f$ is continuously differentiable and

$$
\left(f_{u}(u) w, w\right) \geq-C_{3}|w|^{2}, \forall u, w \in \mathbb{R}^{d},
$$

where $C_{3} \geq 0$ and $f_{u}$ denotes the Jacobian matrix of $u \mapsto f(u)$.

If we consider a bit more general situation where $f=f(x, u)$ is a Carathéodory function, then we can give an example for which at least two weak solutions corresponding to a given initial condition exist. Let $\lambda_{1}>0$ be the first eigenvalue of $-\Delta$ in $H_{0}^{1}(\Omega)$, and $\psi_{1}$ be the corresponding eigenfunction. Without loss of generality we can assume that $\psi_{1}(x)$ $>0$ for any $x \in \Omega$. It is known that $\psi_{1} \in C(\bar{\Omega})$, so that $\max _{x \in \bar{\Omega}}\left|\psi_{1}(x)\right| \leq K$. We put

$$
\begin{gathered}
f(x, u)= \\
\left\{\begin{array}{l}
-\lambda_{1} u-\sqrt{\psi_{1}(x)} \sqrt{u}, u \in[0,1], \\
-\lambda_{1} u-\sqrt{\psi_{1}(x)} u+u^{2}(u-1), u \notin[0,1],
\end{array}\right.
\end{gathered}
$$

and $h \equiv 0$. It is easy to check that $f(x, u)$ satisfies conditions (7)-(8) with $p=4$. Suppose that $a=1$, $u_{0}=0$. Then $u(t, x) \equiv 0$ is a trivial solution of the Cauchy problem (6). For fixed $r \geq 0$ we define

$$
u_{r}(t, x):=\left\{\begin{array}{lc}
0, & 0 \leq t \leq r, \\
\frac{1}{4}(t-r)^{2} \psi_{1}(x), & r \leq t \leq r+\frac{2}{\sqrt{K}} \\
v_{r}(t, x) & t_{r} \leq t \leq T,
\end{array}\right.
$$

where $t_{r}=r+\frac{2}{\sqrt{K}}$ and $v_{r}(t, x)$ is a solution on $\left[t_{r}, T\right]$ with $v_{r}\left(t_{r}, x\right)=\frac{\psi_{1}(x)}{K}$. It is easy to see that the function $u_{\tau}(t, x)$ is a solution of (6) until $\frac{1}{4}(t-$ $r)^{2} \psi_{1}(x) \leq 1$, and it is clear that $\left|u_{r}(t, x)\right| \leq 1$ for all $t \in\left[r, t_{\tau}\right]$. Hence, $u_{r}(t, x)$ is another solution of problem (6). This example shows that conditions (7)-(8) are not enough to give uniqueness.

Let us consider some models for which condition (9) fails.

The complex Ginzburg-Landau equation The complex-valued Ginzburg-Landau equation is the following:

$$
\left\{\begin{array}{c}
\frac{\partial u}{\partial t}=(1+i \eta) \Delta u+R u-(1+i \beta)|u|^{2} u+g(x), \\
\left.u\right|_{\partial \Omega}=0, u(x, 0)=u_{0}(x)
\end{array}\right.
$$

where $u=u(t, x)=u^{1}(t, x)+i u^{2}(t, x)$ for $(x, t) \in$ $\Omega \times[0, T]$ and $g(x)=g^{1}(x)+i g^{2}(x) \in L^{2}(\Omega, \mathbb{C})$ for $\eta, \beta \in \mathbb{R}$ and $R>0$. We assume that $g^{i} \in L^{2}(\Omega)$.

For $v=\left(u^{1}, u^{2}\right)$ and $u=u^{1}+u^{2}$, equation (10) can be written as the real-valued system

$$
\left\{\begin{array}{c}
\frac{\partial v}{\partial t}=\left(\begin{array}{cc}
1 & -\eta \\
\eta & 1
\end{array}\right) \Delta v+\left(\begin{array}{c}
g^{1}(x) \\
g^{2}(x)
\end{array}\right) \\
+\left(\begin{array}{c}
R u^{1}-\left(\left|u^{1}\right|^{2}+\left|u^{2}\right|^{2}\right) \\
\left(u^{1}-\beta u^{2}\right) \\
R u^{2}-\left(\left|u^{1}\right|^{2}+\left|u^{2}\right|^{2}\right) \\
\left(\beta u^{1}+u^{2}\right)
\end{array}\right)
\end{array}\right.
$$

and conditions (7)-(8) hold with $p=(4,4)$. Also, condition (9) holds if $|\beta| \leq \sqrt{3}$ [Chepyzhov \& Vishik, 2002b; p.42].

Hence, if $|\beta| \leq \sqrt{3}$, there exists a unique solution for every initial data in $\left(L^{2}(\Omega)\right)^{2}$. We note that, if $N=1,2$, uniqueness is proved for every $\beta$ [Temam, 1988; p.224]. However, if $N \geq 3$ and we do not assume the condition $|\beta| \leq \sqrt{3}$, then it is not known whether this equation possesses the property of uniqueness of the Cauchy problem or not. 
The Lotka-Volterra system with diffusion We consider the system

$$
\left\{\begin{array}{l}
\frac{\partial u^{1}}{\partial t}=D_{1} \Delta u^{1}+u^{1}\left(a_{1}-u^{1}-a_{12} u^{2}-a_{13} u^{3}\right), \\
\frac{\partial u^{2}}{\partial t}=D_{2} \Delta u^{2}+u^{2}\left(a_{2}-u^{2}-a_{21} u^{1}-a_{23} u^{3}\right), \\
\frac{\partial u^{3}}{\partial t}=D_{3} \Delta u^{3}+u^{3}\left(a_{3}-u^{3}-a_{31} u^{1}-a_{32} u^{2}\right),
\end{array}\right.
$$

with Neumann boundary conditions

$$
\left.\frac{\partial u^{1}}{\partial \nu}\right|_{\partial \Omega}=\left.\frac{\partial u^{2}}{\partial \nu}\right|_{\partial \Omega}=\left.\frac{\partial u^{3}}{\partial \nu}\right|_{\partial \Omega}=0,
$$

where $u^{i}=u^{i}(x, t) \geq 0$ and $a_{i}>0$. Also, the $D_{i}$ are positive constants and $\Omega \subset \mathbb{R}^{3}$.

Conditions (7)-(8) hold for $u \in \mathbb{R}_{+}^{3}$ with $p=$ $(3,3,3)$.

Uniqueness of the Cauchy problem for this system has been proved only if we consider solutions confined in an invariant region (for example, in a parallelepiped $\left.\mathcal{D}=\left\{\left(u^{1}, u^{2}, u^{3}\right): 0 \leq u^{i} \leq k^{i}\right\}\right)$ (see [Marion, 1987] and [Smoller, 1983]). However, in the general case for initial data just in $\left(L^{2}(\Omega)\right)^{3}$ it is still an open problem.

\section{1(C). Parabolic equations with a discontinu- ous nonlinearity}

Let $\Omega \subset \mathbb{R}^{N}$ be a bounded open set with smooth boundary $\partial \Omega$ and consider the differential inclusion

$$
\left\{\begin{array}{c}
\frac{\partial u}{\partial t}-\Delta u+f_{1}(u)-f_{2}(u) \ni h, \text { in }(0, \infty) \times \Omega, \\
\left.u\right|_{\partial \Omega}=0,\left.\quad u\right|_{t=0}=u_{0},
\end{array}\right.
$$

where $h \in L^{2}(\Omega)$ and $f_{i}: \mathbb{R} \rightarrow 2^{\mathbb{R}}$ for $i=1$ and 2 are maximal monotone maps with domain $D\left(f_{i}\right)=$ $\mathbb{R}$ and satisfying

$$
\begin{gathered}
\sup _{y \in f_{2}(s)}|y| \leq K_{1}+K_{2}|s|, \\
\left(y_{1}-y_{2}\right) s \geq\left(-\lambda_{1}+\varepsilon\right) s^{2}-M, \forall y_{i} \in f_{i}(s)
\end{gathered}
$$

for $i=1$ and 2 , where $\lambda_{1}$ is the first eigenvalue of $-\Delta$ in $H_{0}^{1}(\Omega)$, for some $K_{1}, K_{2}, M \geq 0$ and $\varepsilon>0$.

As shown in [Valero, 2001] this equation is a particular case of an abstract differential inclusion generated by a difference of sub-differential maps of proper convex lower semicontinuous functionals
[Otani, 1977], and then we can guarantee the global existence of strong solutions for every $u_{0} \in L^{2}(\Omega)$ (see also [Rossi et al., 2008], where the case of more regular initial data is considered). However, we cannot expect to have uniqueness when the function $f_{2}$ is not continuous. Indeed, consider the following equation

$$
\left\{\begin{array}{cl}
\frac{\partial u}{\partial t}-\frac{\partial^{2} u}{\partial x^{2}} \in H_{0}(u), & \text { on }(0, T) \times(0,1), \\
\left.u\right|_{\partial \Omega}=0, & \left.u\right|_{t=0}=u_{0},
\end{array}\right.
$$

where

$$
H_{0}(u)=\left\{\begin{array}{cc}
-1 & \text { if } u<0 \\
{[-1,1]} & \text { if } u=0 \\
1 & \text { if } u>0
\end{array}\right.
$$

is the Heaviside function. If we consider the initial condition $u_{0}(x)=0$, then obviously $u(t, x) \equiv 0$, for all $t \geq 0$, is a solution, but it is not the only one. In fact, it was shown in [Arrieta et al., 2006] that problem (15) possesses an infinite (but countable) number of stationary points, and for each of these points there exists at least one solution with initial data $u_{0}(x)=0$ converging to it as $t \rightarrow+\infty$. Hence, there exists in fact an infinite number of solutions corresponding to the initial data $u_{0}(x)=0$.

We now consider some models of physical interest.

\section{A model of combustion in porous media} Consider the equation

$$
\left\{\begin{array}{c}
\frac{\partial u}{\partial t}-\frac{\partial^{2} u}{\partial x^{2}}-f(u) \in \lambda H(u-1), \text { in }(0, T) \times(0, \pi), \\
u(0)=u(\pi)=0,\left.\quad u\right|_{t=0}=u_{0},
\end{array}\right.
$$

where $f: \mathbb{R} \rightarrow \mathbb{R}$ is continuous and non-decreasing, $\lambda>0$ and where

$$
H(z)=\left\{\begin{array}{cc}
0, & \text { if } z<0 \\
{[0,1]} & \text { if } z=0 \\
1, & \text { if } z>0
\end{array}\right.
$$

Suppose also that there exist $K_{1} \geq 0,0 \leq K_{2}<$ 1 such that $|f(s)| \leq K_{1}+K_{2}|s|$. This equation models a process of combustion in porous media (see [Feireisl \& Norbury, 1991]) and it is easy to see that conditions (13)-(14) hold [Valero, 2001]. 
A model of conduction of electrical impulses in nerve axons Consider the equation

$$
\left\{\begin{array}{cc}
\frac{\partial u}{\partial t}-\frac{\partial^{2} u}{\partial x^{2}}+u \in H(u-a), & \text { in }(0, T) \times(0, \pi), \\
u(0)=u(\pi)=0,\left.\quad u\right|_{t=0}=u_{0}
\end{array}\right.
$$

where $a \in\left(0, \frac{1}{2}\right)$. In this case $f_{1}(s)=s$ and $f_{2}(s)$ $=H(s-a)$. It is clear that (13)-(14) are satisfied [Valero, 2001]. This inclusion is used as a model of conduction of electrical impulses in nerve axons (see [Terman, 1983, Terman, 1985]).

\section{1(D). A model from climatology}

We now consider a climate energy balance model proposed in [Budyko, 1969], and which has been studied from the dynamical point of view in several works (see e.g. [Díaz et al., 1997, Díaz \& Díaz, 2002, Díaz et al., 2002]). The problem is the following:

$$
\left\{\begin{array}{c}
\frac{\partial u}{\partial t}-\frac{\partial^{2} u}{\partial x^{2}}+B u \in Q S(x) \beta(u)+h(x), \\
(t, x) \in \mathbb{R}^{+} \times(-1,1), \\
u_{x}(-1, t)=u_{x}(1, t)=0, \quad t \in \mathbb{R}^{+}, \\
u(x, 0)=u_{0}(x), \quad x \in(-1,1),
\end{array}\right.
$$

where $B, Q$ and $\varepsilon$ are positive constants, $S, h \in$ $L^{\infty}(-1,1), u_{0} \in L^{2}(-1,1)$ and $\beta$ is a maximal monotone graph in $\mathbb{R}^{2}$, which is bounded, i.e., there exist $m, M \in \mathbb{R}$ such that

$$
m \leq z \leq M, \text { for all } z \in \beta(s), s \in \mathbb{R} .
$$

We also assume that

$$
0<S_{0} \leq S(x) \leq S_{1}, \quad \text { a.e. } x \in(-1,1) .
$$

The unknown $u(t, x)$ represents the averaged temperature of the Earth surface, $Q$ is the so called solar constant, which is the average (over a year and over the surface of the Earth) value of the incoming solar radiative flux, and the function $S(x)$ is the insolation function given by the distribution of incident solar radiation at the top of the atmosphere. When the averaging time is of the order of one year or longer, the function $S(x)$ satisfies (18), for shorter periods we must assume than $S_{0}=0$. The term $\beta$ represents the so called co-albedo function, which can be possibly discontinuous. It represents the ratio between the absorbed solar energy and the incident solar energy at the point $x$ on the Earth surface. Obviously, $\beta(u(x, t))$ depends on the nature of the Earth surface. For instance, it is well known that on ice sheets $\beta(u(x, t))$ is much smaller than on the ocean surface because the white color of the ice sheets reflects a large portion of the incident solar energy, whereas the ocean, due to its dark color and high heat capacity, is able to absorb a larger amount of the incident solar energy.

We point out that this model is very close to (12). In fact, if we consider that the function $f_{2}$ can depend on $x$, then it would be a particular case of that equation. Therefore, we cannot expect to have uniqueness for problem (16) either.

\section{1(E). Wave equation}

Let $\Omega \subset \mathbb{R}^{N}$ be a bounded open set with smooth boundary $\partial \Omega$ and let us consider the nonlinear wave equation

$$
\left\{\begin{array}{c}
\frac{\partial^{2} u}{\partial t^{2}}-\Delta u+\beta \frac{\partial u}{\partial t}+f(u)=0, \\
(t, x) \in(0, T) \times \Omega, \\
\left.u\right|_{\partial \Omega}=0, \\
u(x, 0)=u_{0}(x), \quad \frac{\partial u}{\partial t}(x, 0)=u_{1}(x), x \in \Omega,
\end{array}\right.
$$

where $\beta>0$ and $f: \mathbb{R} \rightarrow \mathbb{R}$ is continuous and satisfies the sign condition

$$
\lim \inf _{|u| \rightarrow \infty} \frac{f(u)}{u}>-\lambda_{1},
$$

with $\lambda_{1}>0$ the first eigenvalue of $-\Delta$ in $H_{0}^{1}(\Omega)$. When $N \geq 3$ we assume that

$$
|f(u)| \leq C_{0}\left(|u|^{\frac{N}{N-2}}+1\right),
$$

for some $C_{0}>0$, while for $N=2$ we suppose that

$$
|f(u)| \leq e^{\theta(u)}
$$

where $\theta(u)$ satisfies

$$
\lim _{|u| \rightarrow \infty} \frac{\theta(u)}{u^{2}}=0 .
$$

These conditions guarantee the existence of at least one globally weak solution for every initial 
data in $H_{0}^{1}(\Omega) \times L^{2}(\Omega)$ [Ball, 2004]. A similar equation on an unbounded cylindrical domain is considered in [Babin, 1995].

In order to obtain uniqueness we need to impose stronger assumptions on $f$. Usually a growth condition on the derivative of $f$ is used (see, for example, [Babin \& Vishik, 1985b, Babin \& Vishik, 1992, Ghidaglia \& Temam, 1985, Ghidaglia \& Temam, 1987, Haraux, 1985, Hale, 1985, Temam, 1988]).

\section{1(F). Phase-field equations}

The phase-field system of equations is a widely studied model which describes the temperature $u$ and the order parameter $\varphi$ in solid-liquid phase boundaries. These equations are very useful for studying materials exhibiting a fine mixture of phases, which is a common phenomenon in many settings. Such processes appear, for example, in the theory of solidification. The problem is the following:

$$
\left\{\begin{array}{l}
\mu \frac{\partial \varphi}{\partial t}-\xi^{2} \Delta \varphi+f(x, \varphi)=2 u+h_{1}(x) \\
\frac{\partial u}{\partial t}+\frac{l}{2} \frac{\partial \varphi}{\partial t}=k \Delta u+h_{2}(x), x \in \Omega, t>0 \\
\left.u\right|_{\partial \Omega}=\left.\varphi\right|_{\partial \Omega}=0, \quad t>0 \\
\left.u\right|_{t=0}=u_{0},\left.\varphi\right|_{t=0}=\varphi_{0}, \quad x \in \Omega
\end{array}\right.
$$

where $\Omega \subset \mathbb{R}^{3}$ is a bounded open subset with smooth boundary $\partial \Omega$ and $\mu, \xi, l$ and $k$ are positive constants, and the functions $f: \Omega \times \mathbb{R} \rightarrow \mathbb{R}$ and $h_{i}: \Omega \rightarrow \mathbb{R}$ for $i=1$ and 2 satisfy:

$$
h_{i} \in L^{2}(\Omega) \text {, }
$$

$f(\cdot, \cdot): \Omega \times \mathbb{R} \mapsto \mathbb{R}$ is Carathéodory, i.e, it is measurable on $x$ and continuous on $r$.

Also, there exists $C \geq 0$ such that

$$
\begin{aligned}
F(x, r) & :=\int_{0}^{r} f(x, s) d s \geq-C, \\
f(x, r) r & -F(x, r) \geq-C, \\
|f(x, r)| & \leq C\left(1+|r|^{3}\right) .
\end{aligned}
$$

Under these conditions existence of globally defined solutions for every initial data in $H_{0}^{1}(\Omega) \times$
$H_{0}^{1}(\Omega)$ can be proved [Kapustyan et al., 2003, Kapustyan et al., 2008].

As in the previous examples to get uniqueness we need to assume stronger assumptions on the nonlinear term as, for example, the monotonicity condition $\frac{\partial}{\partial r} f(x, r) \geq-C$ [Kalantarov, 1991] (see also [Bates \& Zheng, 1992, Brochet et al., 1993, Jiménez-Casas \& Rodríguez-Bernal, 2002]).

In [Rossi et al., 2008] the following quasistationary phase-field model was considered:

$$
\left\{\begin{array}{c}
\frac{\partial}{\partial t}(v+\chi)-\Delta v=0, \\
F^{\prime}(\chi)=v, \quad x \in \Omega, t>0, \\
\left.v\right|_{\partial \Omega}=\left.\frac{\partial \chi}{\partial n}\right|_{\partial \Omega}=0, \quad t>0, \\
\left.v\right|_{t=0}=u_{0},\left.\quad \chi\right|_{t=0}=\varphi_{0}, \quad x \in \Omega,
\end{array}\right.
$$

where $F^{\prime}$ is the Gâteaux derivative of a functional $F$, which is possibly neither smooth nor convex. This arises as a suitable generalization of the quasistationary asymptotics of the phase-field model. One usual choice for $F$ is

$$
F(\chi)=\frac{1}{2} \int_{\Omega}|\nabla \chi|^{2} d x+\frac{1}{4} \int_{\Omega}\left(\chi^{2}-1\right)^{2} d x .
$$

In [Rossi et al., 2008] the existence of solutions is established by using the abstract framework of a parabolic equation generated by the limiting subdifferential of a proper lower semicontinuous (possibly non-convex) functional. Uniqueness for such problems is not known to hold.

Of course, there exist many more examples of systems for which uniqueness can fail or it is not known to be true. Among them we mention differential inclusions of several types (see, among many others, [Aubin \& Cellina, 1984, Otani, 1984, Papageorgiuou \& Papalini, 1996, Tolstonogov \& Umansky, 1992a,

Tolstonogov \& Umansky, 1992b, Vrabie,1997, Yamazaki, 2004]), the Euler equation [Bessaih \& Flandoli, 2000, Constantin, 2007, Shnirelman, 1997], degenerate parabolic equations [Elmounir \& Simonolar, 2000], delay ordinary differential equations with continuous nonlinear term [Hale, 1977, Caraballo et al., 2005], some kinds of three-dimensional Cahn-Hilliard equations [Segatti, 2007, Schimperna, 2007], 
the Boussinesq system [Birnir Svanstedt, 2004, Norman, 1999] or lattice dynamical systems [Morillas \& Valero, 2009].

\subsection{Multi-valued dynamical systems}

One of the most important problems in partial differential equations is the asymptotic behavior of solutions as time goes to infinity. Stability, asymptoptic stability, $\omega$-limit sets and global attractors are key concepts in this theory. Beginning from the pioneering works [Ladyzhenskaya, 1972, Hale \& Lasalle, 1972] the theory of global attractors of infinite dimensional dynamical systems has become a relevant object for investigation. For the application of this classical theory to partial and functional differential equations it was necessary to have global existence and uniqueness of solutions of the Cauchy problem for all initial data of the phase space, as in such a case we are able to define a semigroup of operators. Since then a great number of results concerning existence, structure and fractal dimension of global attractors for a wide class of dissipative systems have been obtained (see e.g. [Temam, 1988, Hale, 1988, Babin \& Vishik, 1992, Ladyzhenskaya, 1991] among many others).

However, as become clear from the previous examples this classical theory cannot be applied to a huge number of equations in which uniqueness of the Cauchy problem either fails or it is not known to be true. In order to work with these problems three main methods have been developed:

1. The method of multi-valued semi-flows;

2. The method of generalized semi-flows;

3. The theory of trajectory attractors.

It is important to point out that the first method for treating non-uniqueness is in fact very old, as it was born many years before the theory of attractors for infinite-dimensional dynamical systems began to be developed in the 70's. We can find multi-valued semi-flows already in the papers [Barbashin, 1948, Barbashin \& Alimov, 1961, Budak, 1952, Bronstein, 1963, Minkevic, 1948] and, later on, for example, also in [Bridgland, 1969a, Bridgland, 1969b,
Bushaw, 1963, Kloeden, 1974, Roxin, 1965a, Roxin, 1965b, Szego \& Treccani, 1969].

However, the application of multivalued semi-flows to the theory of attractors for partial differential equations was given at first in [Babin \& Vishik, 1985a], and extended later in other papers [Babin, 1995, Melnik, 1994, Melnik \& Valero, 1998] (see also the book [Cheban \& Fakeeh, 1992]).

On the other hand, the concept of generalized semi-flow was introduced in [Ball, 1978] and a theory of global attractors for such semigroups was developed in [Ball, 1997, Ball, 2000] (see also [Elmounir \& Simonolar, 2000, Segatti, 2007]).

The theory of trajectory attractors was introduced in the papers [Chepyzhov \& Vishik, 1996, Chepyzhov \& Vishik, 1997, Malek \& Necas, 1996, Sell, 1996].

\section{2(A). Multi-valued semi-flows and general- ized semi-flows}

The method of multi-valued semi-flows and the method of generalized semi-flows are in fact very close and use the same idea: to allow nonuniqueness of the Cauchy problem and to consider the set (or some subset) of its solutions at every moment of time $t$. Hence, a multi-valued analogue of a classical semigroup is considered. The main difference between them is that in the method of multivalued semi-flows, the multi-valued map is considered from the phase space $X$ onto a non-empty subset of the phase space for each moment of time (the set of values attained by the solutions at this time) satisfying some properties similar to the classical ones for semigroups, whereas in the other method, the generalized semi-flow is defined as a set of solutions satisfying some translation and concatenation properties, avoiding in this way the use of multivalued maps. A comparison between these two theories can be found in [Caraballo et al., 2003a].

We shall give a brief review of the main points of these theories and illustrate the results in one of the possible applications.

Let $X$ be a complete metric space with metric $\rho$. Denote by $P(X)(\mathcal{B}(X), C(X), K(X))$ the set of all nonempty (nonempty bounded, nonempty closed, nonempty compact) subsets of $X$. We define the Hausdorff semi-distance for the set $A$ to the 
set $B$ by

$$
\operatorname{dist}(A, B)=\sup _{a \in A} \inf _{b \in B} \rho(a, b) .
$$

Denote by $N_{\varepsilon}(B)=\{y \in X: \operatorname{dist}(y, B)<\varepsilon\}$ an $\varepsilon$-neighborhood of the set $B$.

To begin with, following [Melnik, 1994, Melnik \& Valero, 1998], we define a multi-valued semi-flow $G$ on the phase space $X$ :

Definition 4.1. The (possibly multi-valued) map $G: \mathbb{R}^{+} \times X \rightarrow P(X)$ is called a multi-valued semiflow (m-semi-flow) if the next conditions are satisfied:

1. $G(0, \cdot)=I$ is the identity map;

2. $G\left(t_{1}+t_{2}, x\right) \subset G\left(t_{1}, G\left(t_{2}, x\right)\right)$ for all $t_{1}, t_{2} \in$ $\mathbb{R}^{+}$and $x \in X$,

where $G(t, B):=\cup_{x \in B} G(t, x)$ for $B \subset X$.

It is called strict if, in addition, $G\left(t_{1}+t_{2}, x\right)=$ $G\left(t_{1}, G\left(t_{2}, x\right)\right)$ for all $t_{1}, t_{2} \in \mathbb{R}^{+}$and $x \in X$.

This definition generalizes the concept of semigroup to the multi-valued case.

Let us show how a multi-valued semi-flow is defined, for example, in system (6). We put $X=$ $\left(L^{2}(\Omega)\right)^{d}$. Let $\mathcal{D}\left(u_{0}\right)$ be the set of all globally defined weak solutions such that $u(0)=u_{0}$. Define the map $G$ as

$$
G\left(t, u_{0}\right)=\left\{u(t): u(\cdot) \in \mathcal{D}\left(u_{0}\right)\right\} .
$$

It is proved in [Kapustyan \& Valero, 2006; Lemma 9] that the map $G$ is a strict multi-valued semiflow. The inclusion $G\left(t_{1}+t_{2}, x\right) \subset G\left(t_{1}, G\left(t_{2}, x\right)\right)$ is a consequence of the fact that the translation $u(\cdot+\tau)$ of any weak solution $u(\cdot)$ is a weak solution for any $\tau>0$. Also, the converse inclusion follows from the property of concatenation of solutions: if $u(\cdot), v(\cdot) \in \mathcal{D}\left(u_{0}\right)$, then

$$
z(t)=\left\{\begin{array}{l}
u(t) \quad \text { if } 0 \leq t \leq t_{1}, \\
v\left(t-t_{1}\right) \quad \text { if } t \geq t_{1},
\end{array}\right.
$$

is a new weak solution. We note that the last property can fail sometimes (as we will see later for the example of the three-dimensional Navier-Stokes system). This is the reason of considering in the definition of the map $G$ an inclusion and not an equality.

We observe also that the definition of weak solution for (6) implies certain regularity of the functions included in $\mathcal{D}\left(u_{0}\right)$. Hence, we are not considering all possible solutions of (6) corresponding to a given initial data, but some subset of the solutions. This is important, as in order to obtain some properties of the map $G$ it is necessary a minimal regularity of solutions. For example, we have seen that the weak solutions of (6) are continuous, and this property is crucial in order to prove the compactness of the operator $G(t, \cdot)$ for $t>0$.

Further, let us define the concepts of $\omega$-limit set and global attractor for a general m-semi-flow $G$. For any set $B \subset X$ we put $\gamma_{t}^{+}(B)=\cup_{s \geq t} G(s, B)$ and define the $\omega$-limit set of $B \in \mathcal{B}(X)$ by

$$
\omega(B)=\bigcap_{t \geq 0} \overline{\gamma_{t}^{+}(B)}
$$

It is not difficult to prove that $y \in \omega(B)$ if and only if there exists a sequence $y_{n} \in G\left(t_{n}, B\right)$, where $t_{n}$ $\rightarrow+\infty$ such that $y_{n} \rightarrow y$ in $X$.

A set $A$ is said to be negatively (resp., positively) semi-invariant if $A \subset G(t, A)$ (resp., $G(t, A)$ $\subset A)$ for all $t \geq 0$, and invariant if $A=G(t, A)$ for all $t \geq 0$.

Definition 4.2. The set $\mathcal{A}$ is called a global attractor of the m-semi-flow $G$ if it satisfies the following:

1. $\mathcal{A}$ attracts any $B \in \mathcal{B}(X)$, i.e.,

$$
\operatorname{dist}(G(t, B), \mathcal{A}) \rightarrow 0 \text { as } t \rightarrow+\infty \text {. }
$$

2. $\mathcal{A}$ is negatively semi-invariant.

The main property of a global attractor is thus the attraction property. If we consider for example the semi-flow $G$ given by (26), then this property means that all the weak solutions starting at the bounded set $B$ uniformly converge to the set $\mathcal{A}$ as time goes to $+\infty$. The second property implies for system (6) (using the property of concatenation of solutions) that for any initial data inside the global attractor backward solutions exist.

Usually, the global attractor is assumed to be also compact. However, as this property can fail 
in some applications, we prefer to give this more general definition. We note that if $\mathcal{A}$ is compact, then it is the minimal attracting set, that is, for any closed set $C$ satisfying the attracting property (28) we have $\mathcal{A} \subset C$ and, moreover, $\mathcal{A}$ is unique.

Let us introduce now some definitions that are necessary to obtain a global attractor.

Definition 4.3. The m-semi-flow $G$ is called asymptotically compact if any sequence $\xi_{n} \in$ $G\left(t_{n}, B\right)$ with $t_{n} \rightarrow+\infty$ is precompact in $X$.

In [Melnik \& Valero, 1998] a slightly weaker definition was used. Namely, the property of compactness of $\xi_{n} \in G\left(t_{n}, B\right)$ was assumed to be true only for those sets $B \in \mathcal{B}(X)$ such that $\gamma_{T(B)}^{+}(B)$ $\in \mathcal{B}(X)$ for some $T(B) \in \mathbb{R}^{+}$, i.e., for eventually bounded sets. We think that Definition 4.3 is better, since, in fact, it was supposed later in [Melnik \& Valero, 1998] that all the sets are eventually bounded, so that the property is satisfied for all the bounded sets. Of course, the asymptotic compactness as given in Definition 4.3 implies eventually boundedness of every $B \in \mathcal{B}(X)$, which allows to use less assumptions and make the exposition of the results easier for the reader.

The following result is useful and easy to prove:

Lemma 4.4. If every $B \in \mathcal{B}(X)$ is eventually bounded and the map $G(t, \cdot)$ is compact for some $t>0$ (i.e., it maps bounded sets into precompact ones), then $G$ is asymptotically compact.

Definition 4.5. The set $B_{0}$ is said to be absorbing if for any $B \in \mathcal{B}(X)$ there exists $T(B)$ such that

$$
G(t, B) \subset B_{0}, \quad \forall t \geq T .
$$

Definition 4.6. A multi-valued map $F: X \rightarrow$ $P(X)$ is called upper semicontinuous if for all $u_{0} \in$ $X$ and any neighborhood $O\left(F\left(u_{0}\right)\right)$ there exists $\delta$ $>0$ such that $F(u) \subset O\left(F\left(u_{0}\right)\right)$, as soon as $\rho\left(u, u_{0}\right)$ $<\delta$.

We have:

Theorem 4.7. [Melnik \& Valero, 1998; Theorem 3 and Remark 8] Let $G(t, \cdot): X \rightarrow P(X)$ be upper semicontinuous for any $t \geq 0$ and have closed values (i.e. $G(t, x) \in C(X)$ for all $(t, x))$. Also, assume that $G$ is asymptotically compact and that a bounded absorbing set $B_{0}$ exists. Then a global compact minimal attractor $\mathcal{A}$ exists. Moreover, if $G$ is strict, then the global attractor is invariant.

Remark 4.8. If we assume that the map $G(t, \cdot)$ has closed graph for any $t \geq 0$ and eliminate the assumption of upper semi-continuity, then the above results remains true [Melnik \& Valero, 2008; Lemma 3].

Also, we observe that in fact the conditions given in [Melnik \& Valero, 1998; Theorem 3 and Remark 8] are weaker, since, instead of supposing the existence of a bounded absorbing set, it is assumed only that $G$ is point dissipative, which means that there exists a bounded set $B_{0}$ for which

$$
\operatorname{dist}\left(G(t, x), B_{0}\right) \rightarrow 0 \quad \text { as } t \rightarrow+\infty,
$$

for all $x \in X$. We remark that in this situation if we change the upper semi-continuity of $G(t, \cdot)$ by the assumption of having closed graph, then we need to suppose also that the semi-flow $G$ is strict (see [Melnik \& Valero, 2008]).

The global attractor given in Theorem 4.7 can be characterized as the union of all the $\omega$ limit sets for bounded sets. Indeed, as shown in [Melnik \& Valero, 1998; Theorem 1] the conditions of Theorem 4.7 imply that for any $B \in \mathcal{B}(X)$ the $\omega$-limit set $\omega(B)$ is non-empty, negatively semiinvariant and the minimal closed set attracting $B$. Hence, as the global attractor $\mathcal{A}$ attracts every $B$ $\in \mathcal{B}(X)$, we have $\omega(B) \subset \mathcal{A}$. Also, since $\omega\left(B_{0}\right)$ attracts $B_{0}$, where $B_{0}$ is the absorbing set, then for $\tau \geq T(\mathcal{A})$ and any $\varepsilon>0$ we have

$$
\begin{aligned}
\mathcal{A} & \subset G(t, \mathcal{A}) \subset G(t, G(\tau, \mathcal{A})) \\
& \subset G\left(t, B_{0}\right) \subset N_{\varepsilon}\left(\omega\left(B_{0}\right)\right) \quad \text { if } t \geq T\left(\varepsilon, B_{0}\right),
\end{aligned}
$$

so that $\mathcal{A} \subset \omega\left(B_{0}\right)$. Hence,

$$
\mathcal{A}=\omega\left(B_{0}\right)=\bigcup_{B \in \mathcal{B}(X)} \omega(B) .
$$

One important topological property of the global attractor is its connectivity. For this we need additional assumptions.

The map $x(\cdot): \mathbb{R}^{+} \rightarrow X$ is said to be a trajectory of the m-semi-flow $G$ corresponding to the 
initial condition $x_{0}$ if $x(t+\tau) \in G(t, x(\tau))$ for all $t, \tau \in \mathbb{R}^{+}$and $x(0)=x_{0}$. The m-semi-flow $G$ : $\mathbb{R}^{+} \times X \rightarrow P(X)$ is said to be time-continuous if it is the union of continuous trajectories for all $x_{0} \in$ $X$, i.e.,

$$
G\left(t, x_{0}\right)=\left\{\begin{array}{c}
x(t): x(\cdot) \text { is a trajectory } \\
\text { and } x(\cdot) \in C\left(\mathbb{R}^{+}, X\right)
\end{array}\right\} .
$$

Theorem 4.9. [Melnik \& Valero, 1998; Theorem 5] Let us suppose that the conditions of Theorem 4.7 are satisfied. Assume also that $G$ is a strict time-continuous m-semi-flow with connected values (i.e., $G(t, x)$ is a connected set for every $(t, x))$. If the space $X$ is connected, then the global attractor $\mathcal{A}$ is connected.

Remark 4.10. We can avoid the assumptions that $G$ is strict and time continuous, if we assume the existence of a bounded connected set in $X$ containing the global attractor $\mathcal{A}$ [Amigó et al., 2009; Theorem 4.5]. We note that in order to prove the connectivity is essential to keep the property of upper semi-continuity of $G(t, \cdot)$.

Let us apply these results to the m-semi-flow (26).

First note that the following estimate is true [Kapustyan \& Valero, 2000; p.625]:

$$
\|u(t)\|^{2} \leq e^{-\delta t}\left\|u_{0}\right\|^{2}+K,
$$

for any weak solution of (6), where the constants $\delta$ and $K>0$ are universal. Then a bounded absorbing set exists (for example, a ball in $X$ of radius $\sqrt{1+K}$ centered at 0$)$ and $\gamma_{0}^{+}(B) \in \mathcal{B}(X)$ for all $B \in \mathcal{B}(X)$.

Also, the following lemma holds:

Lemma 4.11. [Kapustyan \& Valero, 2006;

Lemma 2] Let $\left\{u_{n}\right\}$ be an arbitrary sequence of solutions of (6) with $u_{n}(0) \rightarrow u_{0}$ weakly in $H$. Then for any $t_{n} \rightarrow t_{0}$, where $t_{n}, t_{0} \in(0, T]$, there exists a subsequence such that $u_{n}\left(t_{n}\right) \rightarrow u\left(t_{0}\right)$ in $H$, where $u(\cdot)$ is a weak solution of (6) and $u(0)=$ $u_{0}$.

As a consequence of this lemma we have that the map $G(t, \cdot)$ is compact for every $t>0$. Hence,
Lemma 4.4 implies that $G$ is asymptotically compact. Also, it follows that the graph of $G$ is closed and that $G$ has compact values. Finally, by a contradiction argument (see the proof of Corollary 7 in [Kapustyan \& Valero, 2006]) it can be shown that $G(t, \cdot)$ is upper semi-continuous.

On the other hand, it is proved in [Kapustyan \& Valero, 2009a] that the map $G$ has connected values, and the other conditions in Theorem 4.9 are obviously satisfied.

Hence, by Theorems 4.7, 4.9 we have:

Theorem 4.12. The m-semi-flow $G$ generated by (6) possesses a global compact minimal attractor, which is invariant and connected.

Let us consider now the method of generalized semi-flows.

One of the difficulties of the method of multivalued semi-flows is that we have to work with multi-valued maps. This is avoided in this other method. Also, the method of generalized semi-flows has the advantage of working directly with solutions.

Following [Ball, 1997, Ball, 2000] we have:

Definition 4.13. A generalized semi-flow $S$ on $X$ is a family of maps $\varphi:[0, \infty) \rightarrow X$ (called solutions) which satisfy:

(H1) For each $x \in X$ there exists at least one $\varphi \in S$ such that $\varphi(0)=x$;

(H2) (Translation) If $\varphi \in S$ and $\tau \geq 0$, then $\varphi_{\tau} \in$ $S$, where $\varphi_{\tau}(t)=\varphi(\tau+t)$.

(H3) (Concatenation) If $\varphi, \psi \in S$ with $\psi(0)=$ $\varphi\left(t_{1}\right)$, then $\phi \in S$, where

$$
\phi(t)=\left\{\begin{array}{l}
\varphi(t), \quad 0 \leq t \leq t_{1}, \\
\psi\left(t-t_{1}\right), \quad t \geq t_{1} .
\end{array}\right.
$$

(H4) If $\varphi_{j} \in S$ with $\varphi_{j}(0) \rightarrow z$, then there exist $\varphi$ $\in S$ with $\varphi(0)=z$ and a subsequence such that $\varphi_{j}(t) \rightarrow \varphi(t)$, for all $t \geq 0$.

We note here that we can define a multi-valued semi-flow $G: \mathbb{R}^{+} \times X \rightarrow P(X)$ by

$$
G(t, x)=\bigcup_{\substack{\varphi \in S, \varphi(0)=x}} \varphi(t) .
$$


Then conditions $(H 2)-(H 3)$ imply that $G$ is a strict multi-valued semi-flow and condition $(H 4)$ implies that the map $x \mapsto G(t, x)$ is upper semi-continuous and has compact values. Therefore, Definition 4.13 contains stronger conditions than Definition 4.1.

If we consider the reaction-diffusion system (6), then we have seen that the weak solutions satisfy properties $(H 1)-(H 3)$. Also, by Lemma 4.11 we have (H4). Hence, taking $S$ as the union of all weak solutions we obtain a generalized semi-flow.

It is obvious that the associated multi-valued semi-flow defined in (31) coincides with the semiflow given in (26).

We introduce in this case the concepts of $\omega$ limit set and global attractor. For $B \in B(X)$ we set

$$
\omega(B)=
$$

$\left\{\begin{array}{c}z \in X: \text { there exist } \varphi_{j} \in S, \text { with } \varphi_{j}(0) \in B, \\ \text { and } t_{j} \rightarrow+\infty \text { such that } \varphi_{j}\left(t_{j}\right) \rightarrow z .\end{array}\right\}$

It is clear that $\omega(B)$ coincides with the omega-limit set (27) for the associated multi-valued semi-flow given in (31).

Definition 4.14. The set $\mathcal{A}$ is said to be a global attractor for the generalized semi-flow $S$ if the associated multi-valued semi-flow $G$ satisfies the attraction property (28) and $\mathcal{A} \subset G(t, \mathcal{A})$, that is, it is negatively semi-invariant.

In other words, $\mathcal{A}$ is a global attractor for $S$ if it is a global attractor for the associated multivalued semi-flow $G$. In [Ball, 1997, Ball, 2000] it is assumed also that the global attractor has to be compact and invariant, but, as we have commented before, we prefer to give a more general definition and add these properties in the statement of the theorem. The reason is that, although usually in applications these additional properties hold (as is the case of the reaction-diffusion system (6)), sometimes they can fail. When the global attractor is compact, one can check that it is also minimal and unique.

As before in order to obtain the existence of a global attractor we need some previous definitions.

Definition 4.15. The generalized semi-flow $S$ is asymptotically compact if for any sequence $\varphi_{j} \in$
$S$ with $\varphi_{j}(0)$ bounded, any sequence $\varphi_{j}\left(t_{j}\right)$, where $t_{j} \rightarrow+\infty$, is precompact in $X$.

It is clear that $S$ is asymptotically compact if and only if the associated multi-valued semi-flow is asymptotically compact.

Definition 4.16. The generalized semi-flow $S$ is said to be point dissipative if there exists a bounded set $B_{0}$ such that for any $\varphi \in S$ there exists $T(\varphi)$ such that $\varphi(t) \in B_{0}$ for all $t \geq T$.

This definition of point dissipativity is weaker than the one given for multi-valued semi-flows in (29). The difference is that if (29) holds for the associated semi-flow $G$, this implies that all the solutions starting at $\varphi(0)$ entry uniformly in a bounded set $B_{0}$ after some time, that is, there exists a time $T(\varphi(0))$ such that for any solution $\varphi \in S$ we have $\varphi(t) \in B_{0}$ for all $t \geq T$.

The following lemma implies that an asymptotically compact and point dissipative generalized semi-flow satisfies property (29).

Lemma 4.17. [Ball, 2000; Lemma 3.5] Let $S$ be an asymptotically compact and point dissipative generalized semi-flow. Then there exists a bounded set $B_{1}$ such that given any compact $K \subset X$ there exist $\varepsilon=\varepsilon(K)>0, t_{1}=t_{1}(K)>0$ such that $G\left(t, N_{\varepsilon}(K)\right) \subset B_{1}$ for all $t \geq t_{1}$.

It is important to remark that property $(H 4)$ is crucial in the proof of this lemma.

We now state the theorem concerning the existence of a global attractor.

Theorem 4.18. [Ball, 2000; Theorem 3.3] Let $S$ be an asymptotically compact and point dissipative generalized semi-flow. Then there exists a compact global attractor given by

$$
\mathcal{A}=\bigcup_{B \in \mathcal{B}(x)} \omega(B),
$$

which, moreover, is invariant.

With respect to the connectivity of the attractor we have:

Theorem 4.19. [Ball, 2000; Corollary 4.3] Assume the conditions of Theorem 4.18. Let $X$ be 
a connected space. Assume that the operator $G$ has connected values and that every $\varphi \in S$ belongs to $C((0, \infty) ; X)$. Then the global attractor $\mathcal{A}$ is connected.

We note that the definition of the generalized semi-group $S$ implies that the associated semi-flow $G$ is strict and that the map $G(t, \cdot)$ is upper semicontinuous. Also, it follows from the fact that every $\varphi \in S$ belongs to $C((0, \infty) ; X)$ that $G$ can be represented as the union of trajectories which are continuous on $t \in(0, \infty)$. Thus the conditions imposed in this theorem are almost the same as in Theorem 4.9 (in fact, the only difference is that there it is assumed that the trajectories are continuous on $t \in[0, \infty)$.

Let us apply these results to equation (6). As we have seen before it follows from (30) and Lemma 4.11 that $S$ is an asymptotically compact and point dissipative generalized semi-flow. Also, as the weak solutions of (6) are continuous, it is clear that every $\varphi \in S$ belongs to $C((0, \infty) ; X)$. The space $X=$ $\left(L^{2}(\Omega)\right)^{d}$ is connected and we have seen that $G$ has connected values.

Hence, by Theorems 4.18, 4.19 we have:

Theorem 4.20. The generalized semi-flow $S$ generated by (6) possesses a global compact minimal attractor, which is invariant and connected.

Remark 4.21. Since the associated multi-valued semi-flow $G$ coincides with the semi-flow defined in (26) and the global attractor is unique, we see that the global attractors given in Theorems 4.12, 4.20 are the same.

Remark 4.22. Under some conditions the global attractors given in Theorems 4.7, 4.18 are described as the union of all bounded complete trajectories of the semi-flow [Ball, 2000, Kapustyan et al., 2008, Simsen \& Gentile, 2008].

In conclusion, we can say that these two methods are very close and allow rather similar results to be obtained. The main difference is that in the method of generalized semi-flows several extra conditions are included in the definition, whereas in the method of multi-valued semi-flows such conditions are added when they become necessary. On the other hand, the method of generalized semi-flows allows to use a weaker definition of point dissipativity.

These approaches have been very useful and productive and have allowed results about existence and properties of attractors to be obtained for a wide class of dissipative systems without uniqueness. For example, these results have been applied fruitfully to reactiondiffusion equations [Iovane \& Kapustyan, 2006, Kapustyan, 2002, Kapustyan \& Shkundin, 2003, Kapustyan \& Valero, 2006,

Morillas \& Valero, 2005], the three-dimensional Navier-Stokes equation [Ball, 1997, Ball, 2000, Cheskidov, 2006, Cheskidov \& Foias, 2006, Kapustyan et al., 2007, Kapustyan \& Valero, 2007, Kloeden \& Valero, 2007, Rosa, 2006], the wave equation [Ball, 2004, Horban \& Stanzhyts'kyi, 2008,

Iovane \& Kapustyan, 2005, Wang \& Zhou, 2007], differential inclusions [Carvalho \& Gentile, 2003, Cheban \& Mammama, 2006,

Kapustyan \& Valero, 2000,

Kenmochi \& Yamazaki, 2001, Kloeden \& Li ,2005, Li et al., 2008, Melnik \& Valero, 1998, Segatti, 2006, Valero, 2000, Valero, 2001, Yamazaki, 2004], delay ordinary differential equations [Caraballo et al., 2005, Caraballo et al., 2007], degenerate parabolic equations [Elmounir \& Simonolar, 2000], lattice systems [Morillas \& Valero, 2009], the phase-field equation [Kapustyan, 1999, Kapustyan et al., 2003, Morillas \& Valero, 2008, Rossi et al., 2008, Valero, 2005] or the Cahn-Hilliard equation [Segatti, 2007, Schimperna, 2007]. See also the monographs [Kapustyan et al., 2008, Cheban, 2004].

Of course, in the case of uniqueness of solutions all results in the two methods coincide with the classical ones.

\section{2(B). The method of trajectory attractors}

In order to avoid the problem of nonuniqueness of the Cauchy problem in the papers [Chepyzhov \& Vishik, 1996, Chepyzhov \& Vishik, 1997,

Chepyzhov \& Vishik, 2002a, Malek \& Necas, 1996, Sell, 1996] (see also [Chepyzhov \& Vishik, 2002b, 
Malek \& Prazak, 2002]) a new method for studying the asymptotic behavior of solutions was proposed, which is now known as the method of trajectory attractors.

The main idea of this method is the following. Let us consider some collection of solutions of an equation, denoted by $\mathcal{K}^{+}=\{\varphi(s), s \geq 0\}$. These solutions belong to some topological space $W$, which usually is a space with some local convergence topology on any interval $\left[t_{1}, t_{2}\right] \subset \mathbb{R}^{+}$. If we assume that for any $\varphi \in \mathcal{K}^{+}$we have that $\varphi_{\tau}(\cdot)$ $:=\varphi(\cdot+\tau) \in K^{+}$for all $\tau \geq 0$, then the translation semi-group $S(t, \cdot): W \rightarrow W$ given by

$$
S(t) \varphi(\cdot)=\varphi_{t}(\cdot)=\varphi(\cdot+t)
$$

is well defined.

This method has the advantage that a global attractor for such problems as the threedimensional Navier-Stokes equations can be constructed, whereas this question remains still open using the theory of multi-valued semi-flows or generalized semi-flows. However, as pointed out in [Ball, 2000], the disadvantage is that the direct connection with the evolution of the system in the physical phase space is lost.

In the cited papers different approaches are used in order to construct a trajectory attractor, i.e., a global attractor for the translation semigroup. We shall describe briefly the methods given in [Chepyzhov \& Vishik, 2002a, Sell, 1996] and their application to the three-dimensional Navier-Stokes system (4).

In [Sell, 1996] it is considered that $W$ is a complete metric space. Then the standard theory of attractors for classical semi-groups is used.

On the other hand, in [Chepyzhov \& Vishik, 2002a] a bit different approach is used. Let us take some Banach spaces $E$ and $E_{0}$ such that $E \subseteq E_{0}$ with continuous embedding (it is possible to have $E=E_{0}$ ), and let

$$
W=L^{\infty}\left(\mathbb{R}^{+} ; E\right) \cap C\left(\mathbb{R}^{+} ; E_{0}\right) .
$$

We note that if $\varphi(\cdot) \in W$, then $\varphi(\cdot) \in C_{w}\left(\mathbb{R}^{+} ; E\right)$ (i.e., it is continuous with respect to the weak topology of $E$ ) and

$$
\|\varphi(t)\|_{E} \leq\|\varphi\|_{L^{\infty}\left(\mathbb{R}_{+} ; E\right)}, \forall t \geq 0 .
$$

The convergence in $C\left(\mathbb{R}_{+} ; E_{0}\right)$ is given by

$$
f_{n}(\cdot) \rightarrow f(\cdot) \text { in } C\left(\mathbb{R}^{+} ; E_{0}\right) \Longleftrightarrow
$$

$\Pi_{M} f_{n}(\cdot) \rightarrow \Pi_{M} f(\cdot)$ in $C\left([0, M] ; E_{0}\right), \forall M>0$,

where $\Pi_{M}$ is the operator of restriction on $[0, M]$.

We observe that the translation semigroup is continuous in the space $C\left(\mathbb{R}^{+} ; E_{0}\right)$ [Chepyzhov \& Vishik, 2002a; Proposition 1.1].

Definition 4.23. The set $U \subset \mathcal{K}^{+}$is called a trajectory attractor (with respect to the space of trajectories $\mathcal{K}^{+}$in the topology $C\left(\mathbb{R}^{+} ; E_{0}\right)$, if:

1. $U$ is compact in $C\left(\mathbb{R}^{+} ; E_{0}\right)$ and bounded in $L^{\infty}\left(\mathbb{R}^{+} ; E\right)$;

2. $U$ is invariant, that is, $T(t) U=U$ for all $t \geq$ 0

3. $U$ is an attracting set, that is, for every set $B$ $\subset \mathcal{K}^{+}$, bounded in $L^{\infty}\left(\mathbb{R}^{+} ; E\right)$, we have that for any $M>0$,

$$
\operatorname{dist}_{C\left([0, M] ; E_{0}\right)}\left(\Pi_{M} T(t) B, \Pi_{M} U\right) \rightarrow 0,
$$

as $t \rightarrow \infty$.

The function $\varphi(\cdot) \in L^{\infty}(\mathbb{R} ; E) \cap C\left(\mathbb{R} ; E_{0}\right)$ is called a complete trajectory for $\mathcal{K}^{+}$, if

$$
\Pi_{+} \varphi_{h}(\cdot) \in \mathcal{K}^{+}, \forall h \in \mathbb{R}
$$

where $\varphi_{h}(s)=\varphi(s+h)$ and $\Pi_{+}$is the operator of restriction on $[0, \infty)$. Let $\mathbb{K}$ be the union of all complete trajectories for $\mathcal{K}^{+}$.

Theorem 4.24. [Chepyzhov \& Vishik, 2002a] If there exists an attracting set $P \subset \mathcal{K}^{+}$, which is compact in $\mathbb{C}\left(\mathbb{R}^{+} ; E_{0}\right)$ and bounded in $L^{\infty}\left(\mathbb{R}^{+} ; E\right)$, then there exists a trajectory attractor $U \subseteq P$ and

$$
U=\Pi_{+} \mathbb{K} .
$$

In the case where uniqueness of the Cauchy problem holds (and then a semi-group $S$ can be given), in [Chepyzhov \& Vishik, 2002a; Corollary 2.1 it is shown a relation between the trajectory attractor $U$ and the global attractor of the semigroup $S$. Namely, assume that for any $z \in E$ there exists a unique trajectory $\varphi(\cdot) \in \mathcal{K}^{+}$such that $\varphi(0)$ $=z$. Then the semi-group given by $S(t, z)=\varphi(t)$ for all $t \geq 0$ is well defined. Then if we assume also that the set $\cup_{t \geq 0} S(t, B)$ is bounded in $E$ for any set $B$, which is bounded in $E$, then under the assumptions of Theorem 4.24 the set $\mathcal{A}=U(0)$ is 
a global $\left(E, E_{0}\right)$-attractor for $S$. This means that the set $\mathcal{A}$ is bounded in $E$, compact in $E_{0}$, it is invariant (i.e., $S(t, \mathcal{A})=\mathcal{A}$ for all $t \geq 0$ ) and attracts every bounded set of $E$ in the metric of $E_{0}$.

This result can be generalized to the case of non-uniqueness, that is, when we assume that for any $z \in E$ there exists at least one trajectory $\varphi(\cdot) \in \mathcal{K}^{+}$such that $\varphi(0)=z$. In such a case we define a multi-valued semi-flow $G$ given by $G(t, z)=\left\{\varphi(t): \varphi(\cdot) \in \mathcal{K}^{+}\right.$such that $\left.\varphi(0)=z\right\}$ and assuming that $\cup_{t \geq 0} G(t, B)$ is bounded in $E$ for any set $B$, bounded in $E$, we can obtain the same result [Kapustyan \& Valero, 2009a].

Let us consider system (4). In the paper [Chepyzhov \& Vishik, 2002a] the set $\mathcal{K}^{+}$is the union of all weak solutions of (4) satisfying a suitable energy inequality. We take $E=H$ and $E_{0}=H^{-\delta}$, where $H$ is given in (5) and $H^{\sigma}$ stands for the scale of Hilbert spaces corresponding to the Hilbert space $H$. Then it is proved that an attracting set $U$, which is compact in $C\left(\mathbb{R}^{+} ; H^{-\delta}\right)$ and bounded in $L^{\infty}\left(\mathbb{R}^{+} ; H\right)$, exists. Therefore, Theorem 4.24 implies the existence of a trajectory attractor.

Now let us consider Sell's approach [Sell, 1996]. There the set $\mathcal{K}^{+}$is the union of all weak solutions of (4) satisfying some regular assumptions and suitable dissipation and energy inequalities, as well. The space $W$ is defined as the set $\mathcal{K}^{+}$endowed with the topology of $L_{l o c}^{2}(0, \infty ; H)$, which is a complete metric space. Then, using the classical theory of attractors for semi-groups [Ladyzhenskaya, 1991, Sell \& You, 1995], it is proved that the translation semi-group has a compact global attractor $U$ in $W$, that is, $U$ is compact in $W$, it attracts every bounded set of $W$ and is invariant $(S(t) U=U$ for all $t \geq 0)$.

As the two previous methods, the method of trajectory attractors has been fruitfully applied to different partial differential equations. For example we can cite applications to the three-dimensional Navier-Stokes equation and related systems [Chepyzhov \& Vishik, 1997, Chepyzhov \& Vishik, 2002a, Cutland, 2005, Feireisl, 2000, Flandoli \& Schmalfuß, 1999, Malek \& Necas, 1996, Malek \& Prazak, 2002, Norman, 1999, Sell, 1996, Vorotnikov \& Zvyagin, 2007], diffusion systems [Chepyzhov \& Vishik, 1996, Efendiev \& Zelik, 2001], differential inclusions [Hetzer, 2001, Hetzer \& Tello, 2002], the wave equations [Chepyzhov \& Vishik, 1997] or equations with delay [Chepyzhov et al., 2006].

\subsection{Technical difficulties and open prob- lems in multi-valued dynamical sys- tems}

In the previous subsection we reviewed the main points of two different theories for multi-valued dynamical systems. It is natural to ask about additional technical problems which arise if we compare them with the classical case for which uniqueness holds.

\section{3(A). Asymptotic compactness and the 3D Navier-Stokes system}

One of the difficulties which appears as a consequence of the lack of uniqueness is that, unlike the case with uniqueness, there are strong restrictions in the kind of estimates that we can obtain. This occurs because, usually, we do not have a regular approximation for each solution of the equation and, as a result, we cannot obtain formally the necessary estimates and justify them through suitable approximations. We are only able to obtain the estimates that the regularity properties of the solutions allow.

In particular, this problem arises when we need to prove the property of asymptotic compactness of a multi-valued semi-flow or a generalized semigroup which, as we have seen, it is crucial in order to prove the existence of a global attractor.

For example, let us consider the reactiondiffusion system (6). In this case the asymptotic compactness of the multi-valued semi-flow (or the single-valued semi-group if we have uniqueness) is proved by checking that the map $G(t, \cdot)$ is compact for any $t>0$. If the monotonicity assumption (9) holds (so that we have uniqueness), then for any weak solution we can obtain (formally) an estimate of the type

$$
\|u(t)\|_{V} \leq C\left(t,\left\|u_{0}\right\|_{H}\right), \forall t>0,
$$

where $V=\left(H^{1}(\Omega)\right)^{d}$ or $\left(H_{0}^{1}(\Omega)\right)^{d}$ and $H=$ $\left(L^{2}(\Omega)\right)^{d}$, and $C\left(t,\left\|u_{0}\right\|_{H}\right) \rightarrow \infty$ as $t \rightarrow 0^{+}$ [Chepyzhov \& Vishik, 1996; p.67]. Then using the compact embedding $V \subset H$ we obtain that the 
semi-group $S(t)$ is compact for any $t>0$. Since the solution is unique, we can justify this result as follows: we take a suitable regular approximation sequence for which the estimate is correct (for example, a Galerkin approximative sequence) and then passing to the limit we obtain the estimate for the weak solutions of the equation.

When condition (9) is not satisfied, this argument fails, as we cannot state that every weak solution can be approximated by a Galerkin sequence: we just can say that at least one weak solution can be approximated. Hence, we cannot obtain (34) for all weak solutions. The same problem appear in other equations as the phase-field system (21) or the wave equation (19), although it is worth to point out that in some multi-valued systems we have enough regularity in order to obtain estimates of the kind (34), as for example equation (12) [Valero, 2001].

To avoid this problem two appropriate tools have been used in several papers: the method of the energy equation and the monotonicity method. For example, the first one has been applied in [Ball, 2004] to the wave equation (19) and in [Morillas \& Valero, 2005] to the reactiondiffusion system (6) in unbounded domains, and the second one in [Kapustyan et al., 2003] to the phase-field system (21) and to the reactiondiffusion system (6) in [Morillas \& Valero, 2005, Kapustyan \& Valero, 2006]. We note that the method of the energy equation has been also successfully used in many papers for equations with uniqueness of solutions (see e.g. [Ghidaglia, 1994, Lu \& Wang, 2001, Moise et al., 1998, Rosa, 1998, Wang, 1999]).

The key idea in these methods is the following. Let the phase space $X$ be a Hilbert space (of course, more general situations are possible). In such spaces if $x_{n} \rightarrow x$ weakly and $\left\|x_{n}\right\| \rightarrow\|x\|$, then $x_{n} \rightarrow x$ strongly. If we consider the property of asymptotic compactness, then we take a sequence $y_{n} \in G\left(t_{n}, x_{n}\right)$ with $x_{n} \in \mathcal{B}(X)$, where $G$ is the multi-valued semi-flow generated by the equation, and we need to prove that $\left\{y_{n}\right\}$ is precompact. Assuming that the a priori estimates of the equation give us that this sequence is bounded, we obtain passing to a subsequence that $y_{n} \rightarrow y$ weakly in $X$. Then

$$
\|y\| \leq \liminf _{n \rightarrow \infty}\left\|y_{n}\right\|
$$

and in order to obtain $\left\|y_{n}\right\| \rightarrow\|y\|$ (so $y_{n} \rightarrow y$ strongly) it is enough to show that

$$
\limsup _{n \rightarrow \infty}\left\|y_{n}\right\| \leq\|y\| .
$$

Hence, estimates in more regular spaces are avoided. The two methods diverge in the way of proving the last inequality, as one can check in the cited papers.

We observe that these methods fail in the case of the three-dimensional Navier-Stokes system (4), so that the problem of obtaining the property of asymptotic compactness for this equation remains open so far. For example, the method of monotonicity does no work because the weak solutions are not known to be continuous (just weakly continuous) and this property is necessary in this method. Nowadays, if we consider an arbitrary external force $f \in H$, then the question about the asymptotic compactness for the solutions of (4) is open. In the papers [Ball, 2000, Cheskidov \& Foias, 2006, Kapustyan \& Valero, 2007, Rosa, 2006] some conditional results have been obtained in this direction (that is, under assumptions that are not known to hold). On the other hand, in the papers [Birnir Svanstedt, 2004, Bondarevski, 1997] under some quite restrictive conditions on the external force $f$, it is shown that for every bounded set $B$ all the weak solutions starting at $B$ become regular after a time $T(B)$, and in this way it is proved the existence of a global attractor.

On the other hand, there can be also difficulties in obtaining an absorbing set. Although this problem does not appear in reaction-diffusion systems or in the phase-field system (as we have enough regularity to obtain similar estimates as in the case of uniqueness), again some extra effort is needed for the three-dimensional Navier-Stokes system. If we multiply equation (4) by $u$ and make some standard operations, one can obtain (formally) an estimate in the norm of the space $H$ of the kind:

$$
\begin{gathered}
\|u(t)\|^{2} \leq e^{-\nu \lambda_{1} t}\left(\|u(0)\|^{2}-\frac{1}{\nu^{2} \lambda_{1}^{2}}\|f\|^{2}\right) \\
+\frac{1}{\nu^{2} \lambda_{1}^{2}}\|f\|^{2}, \forall t \geq 0 .
\end{gathered}
$$

However, we cannot state that this holds for every weak solution, and just for the one that was obtained via the Galerkin approximations. Neverthe- 
less, estimate (35) can be proved if we take a suitable subset of the set of weak solutions. Namely, in [Ball, 2000] it is taken only the subset of weak solutions satisfying the following energy inequality:

$V(u(t)) \leq V(u(s)), \forall t \geq s$, a.a. $s>0$ and $s=0$,

where

$$
\begin{aligned}
& V(u(t)):=\frac{1}{2}\|u(t)\|_{H}^{2} \\
& +\nu \int_{0}^{t}\|u(r)\|_{V}^{2} d r-\int_{0}^{t}(f(r), u(r)) d r .
\end{aligned}
$$

For such solutions (35) holds [Ball, 2000] and then a bounded absorbing set exists.

However, such a choice of solutions has another problem. If we define the translation $u_{\tau}(t)=$ $u(t+\tau)$, then property (36) fails for $u(\cdot)$ at $s=0$. Thus, property (H2) of the definition of generalized semi-flow is not satisifed. Also, for the associated multi-valued map $G(t, \cdot)$ the key property $G(t+s, x) \subset G(t, G(s, x))$ fails.

We could try to modify inequality (36) in the following way:

$$
V(u(t)) \leq V(u(s))
$$

holds for all $t \geq s$ and a.a. $s>0$. Then the map $u_{\tau}(t)$ also satisfies this property and a multi-valued semi-flow can be correctly defined. However, in such case we cannot prove (35). One can check that the following holds [Kapustyan et al., 2008; Lemma 5.29]:

$$
\begin{gathered}
\|u(t)\|^{2} \leq e^{-\nu \lambda_{1}(t-s)}\left(\|u(s)\|^{2}-\frac{1}{\nu^{2} \lambda_{1}^{2}}\|f\|^{2}\right) \\
+\frac{1}{\nu^{2} \lambda_{1}^{2}}\|f\|^{2}, \forall t \geq s \text { and a.a. } s>0,
\end{gathered}
$$

but this is not enough to obtain an absorbing set. Also, we note that in this case the concatenation of two solutions do not have to satisfy necessarily (37) and then we cannot state that the semi-flow is strict. Moreover, property (H3) fails and a generalized semi-flow cannot be defined in this case.

We observe that, without defining a multivalued semiflow, the uniform convergence of the weak solutions starting in a bounded set to the universal attractor (which consists of points in bounded complete trajectories) is proved in [Foias et. al, 2001].

Finally, a multi-valued semi-flow is defined in [Kapustyan \& Valero, 2007,
Kapustyan et al., 2008] with solutions satisfying (37) and having a bounded absorbing set by restricting the phase space to a ball of radius $R$ $\geq R_{0}$, where $R_{0}^{2}=\frac{1}{\nu^{2} \lambda_{1}^{2}}\|f\|^{2}$. Namely, put $B_{R}=$ $\{u \in H:\|u\| \leq R\}$ and for any $R \geq R_{0}$ define the multi-valued map $G^{R}: \mathbb{R}^{+} \times B_{R} \rightarrow P\left(B_{R}\right)$ as

$$
G^{R}\left(t, u_{0}\right):=
$$

$\left\{\begin{array}{c}u(t): u(\cdot) \text { is a globally defined weak solution } \\ \text { with } u(0)=u_{0} \text { such that }(37) \text { holds and } \\ \|u(r)\| \leq R, \text { for all } r \geq 0 .\end{array}\right\}$

This map is well defined since at least one such solution exists [Kapustyan \& Valero, 2007, Kapustyan et al., 2008] and, as the phase space is bounded, $G^{R}$ has a bounded absorbing set. Then the existence of a global attractor can be proved in the space $B_{R}$ endowed with the weak topology of $H$. We note that in [Kapustyan \& Valero, 2007] inequality (38) is used also in the definition of $G^{R}$, but this is not necessary as (37) implies (38).

\section{3(B). The structure and fractal dimension of attractors of multi-valued dynamical systems}

We have seen so far that a lot of results have been proved about the existence of global attractors for different multi-valued dynamical systems. Moreover, some topological properties such as its connectedness has been studied. However, not much is known about the structure of these attractors and also about their fractal dimension. Comparing with the classical single-valued semi-groups these problems are much more difficult to solve in the multivalued case.

Let us consider the simplest structure of a global attractor. If we consider a continuous classical semi-group having a global compact connected attractor and a Lyapunov (also called energy) function, then, when the set of stationary points is finite, it is well known that the attractor consists of the set of stationary points and all the bounded complete trajectories (heteroclinic connections) joining them [Ladyzhenskaya, 1990]. In particular applications we need to determine which connections exist, as this will give us a full description of the attractor. The Chafee-Infante equation is a very well known model in which this problem has been solved. In this equation the number of 
stationary points is finite (this number depends on a parameter) and a global compact connected attractor, together with a Lyapunov function, exist. Moreover, the set of stationary points is ordered by the Lyapunov function $V$. In [Henry, 1985] a complete description of this attractor is given by showing that the two stationary points with less energy are stable, whereas the other ones are unstable, and that the necessary and sufficient condition for the existence of an heteroclinic connection from a point $v$ to a point $u$ is that $V(v)>V(u)$, i.e., if the energy of $v$ is greater than the energy of $u$. More general and complicated cases can be found, for example, in [Brunovsky \& Fiedler, 1989, Fiedler \& Rocha, 1996].

Can we obtain such results in multi-valued systems? It is possible, of course, but it is much more difficult than in the single-valued case. For example, if we consider the property of stability of stationary points, then one can see that the method of linearization is not applicable, as the non-linear terms of equation as (6), (12), (19) or (21) are not differentiable. Hence, one of the main tools for studying stability in non-linear differential equations cannot be used in multi-valued systems. Nevertheless, some partial results are known so far.

In [Arrieta et al., 2006] it is studied the following differential inclusion

$$
\left\{\begin{array}{c}
\frac{\partial u}{\partial t}-\frac{\partial^{2} u}{\partial x^{2}}-H_{0}(u) \ni 0, \text { on }(0,1) \times(0, T), \\
u(0, t)=u(1, t)=0, \\
u(x, 0)=u_{0}(x),
\end{array}\right.
$$

where

$$
H_{0}(u)=\left\{\begin{array}{l}
-1, \quad \text { if } u<0 \\
{[-1,1], \quad \text { if } u=0} \\
1, \quad \text { if } u>0,
\end{array}\right.
$$

is the Heaviside function. This inclusion is a particular case of (12). First, it was proved that equation (39) has an infinite, but countable, number of equilibria $v_{0}=0, v_{1}^{ \pm}, v_{2}^{ \pm}, \ldots, v_{k}^{ \pm}, \ldots$, which can be ordered using a natural energy (or Lyapunov function) $E(u)$ :

$$
\begin{aligned}
E\left(v_{1}^{+}\right) & =E\left(v_{1}^{-}\right)<E\left(v_{2}^{+}\right)=E\left(v_{1}^{2}\right)<\ldots \\
\ldots<E\left(v_{k}^{+}\right) & =E\left(v_{k}^{-}\right)<\ldots<E\left(v_{0}\right) .
\end{aligned}
$$

Then, it is shown that $v_{1}^{ \pm}$are asymptotically stable fixed points, and all the other ones are unstable. The fixed point $v \equiv 0$ possesses the following remarkable property: for any fixed point $v_{k}$ different from 0 there exists a solution, $u(t)$, with initial value $u(0)=0$, such that $u(t)$ converges to $v_{k}$ as $t \rightarrow+\infty$. We note that the existence of a Lyapunov function implies in this case, as occurs for the Chafee-Infante problem, that the global attractor can be described completely by the equilibria and the heteroclinic connections between them. The natural question is then to establish which connections actually exist.

The global attractor of (39) is approximated by a sequence of attractors $\mathcal{A}_{\varepsilon_{n}}$ corresponding to an approximating sequence of Chafee-Infante problems, for which, as we have seen, all existing connections are known. The natural conjecture is that the connections are the same when we pass to the limit case, that is, that a connection exists from the fixed point $v$ to the fixed point $v^{*}$ if and only if $E(v)$ $>E\left(v^{*}\right)$. Of course, since the energy is decreasing along the trajectories, no connections can exist if $E(v) \leq E\left(v^{*}\right)$. It is natural to expect that (39) is equivalent to a Chafee-Infante problem that has undergo all the typical bifurcation cascade of these type of problems, and thus all connections should be present. In [Arrieta et al., 2006] some of these connections are established, but the complete description of the attractor remains an open problem.

On the other hand, in [Díaz et al., 1997, Díaz et al., 2002] the authors show that equation (16) also has an infinite, but countable, number of equilibria. Probably, the structure of the attractor of this equation is the same as for (39).

For other problems like (6), (19) or (21) nothing is known in this direction.

Finally, let us consider the problem of obtaining estimates of the fractal and Hausdorff dimensions of the global attractor. This question has been studied fruitfully and widely for nonlinear single-valued semi-groups and in this way deep results have been proved in a lot of papers. To mention some of them, for example, estimates of the fractal and Hausdorff dimensions depending on the physical parameter of the equation were proved for the twodimensional Navier-Stokes system, for reactiondiffusion systems and for the wave equation (see e.g. [Ladyzhenskaya, 1982, Temam, 1988]). 
In the multi-valued case almost nothing has been done in this direction. Some abstract theorems concerning the fractal dimension of multivalued semi-flows can be found in [Melnik, 1998] (see also [Kapustyan et al., 2008]), a result which was applied in [Valero, 2000] to a differential inclusion. However, the conditions imposed in [Melnik, 1998] are too strong and, in fact, in the application given in [Valero, 2000] the multi-valued semi-flow is single-valued when we restrict it to the global attractor. The main difficulty in the multi-valued case is that the technique used in the single-valued one is not suitable due to the fact that either a Lipschitz property of the semi-group [Ladyzhenskaya, 1982, Ladyzhenskaya, 1990] or a strong differentiability property [Temam, 1988] is required. In particular, such properties do not hold for multi-valued semi-flows.

Nevertheless, from our point of view there is not an objective reason to think that the global attractors of problems like (6), (19) or (21), for example, do not satisfy similar estimates of the fractal dimension as in the case of uniqueness. Thus, we are convinced that we need just a new technique in which the semi-flow would not need to be either Lipschitz or differentiable.

On the other hand, there are multi-valued problems in which we could expect to have an infinite dimensional global attractor. An example is given in [Valero, 2000] for a differential inclusion. Also, we think that, probably, this is true also for the attractor of equation (39). The heuristic reason is the following: we have seen that for any fixed point $v_{k}$ different from 0 there exists a solution, $u(t)$, with initial value $u(0)=0$, such that $u(t)$ converges to $v_{k}$ as $t \rightarrow+\infty$, and in some sense this means (as the number of fixed points $v_{k}$ is infinite) that the unstable manifold of 0 (if it exists, of course) is infinite-dimensional. Also, we note that the global attractor of (39) is approximated by a sequence of attractors of Chafee-Infante problems with growing dimension. The same could be true for equation (16).

In conclusion, we state the following relevant open problems in the theory of multi-valued dynamical systems:

1. Obtain the existence of a global compact attractor for the three-dimensional Navier-
Stokes system (4) in the phase space $H$ for an arbitrary external force $f \in H$.

2. Describe the structure of the global attractor in some applications.

3. Study the stability of stationary points in some applications.

4. Develop a new method for obtaining estimates of the fractal and Hausdorff dimensions.

\section{Non-autonomous and random dynamical systems}

Undoubtedly, non-autonomous and random systems are also of great importance and interest as they appear in many applications to natural sciences. On some occasions, some phenomena are modeled by nonlinear evolutionary equations which do not take into account all the relevant information of the real systems. Instead some neglected quantities can be modeled as an external force which in general becomes time-dependent (sometimes periodic, quasi-periodic or almost periodic due to seasonal regimes) or even can contain random/stochastic features (also called noise).

In the finite-dimensional framework (i.e. for non-autonomous ordinary differential equations in $\mathbb{R}^{N}$ ) the long-time behaviour of non-autonomous dynamical systems has been widely studied by means of the theory of skew-product flows (see the pioneering works [Miller, 1965] and [Sell, 1996]). However, most of the progress in the infinitedimensional context, i.e., for non-autonomous partial differential equations and specially for systems appearing in mathematical physics, has been done during the last two decades.

The first attempts to extend the notion of global attractor to the non-autonomous case led to the concept of the so-called uniform attractor (see [Chepyzhov \& Vishik, 1996]). It is remarkable that the conditions ensuring the existence of the uniform attractor parallel those for autonomous systems. To this end, non-autonomous systems are lifted in [Vishik, 1992] to autonomous ones by expanding the phase space. Then, the existence of uniform attractors relies on some compactness property of the solution operator associated to the system. However, one disadvantage of this uniform 
attractor is that it needs not be "invariant" unlike the global attractor for autonomous systems. At the same time, the theory of pullback (or cocycle) attractors has been developed for both the nonautonomous and random dynamical systems (see [Crauel et al., 1995, Langa \& Schmalfuß, 2004, Kloeden \& Siegmund, 2005,

Kloeden \& Schmalfuß, 1998, Schmalfuß, 2000]), and has shown to be very useful in the understanding of the dynamics of non-autonomous dynamical systems. In this case, the concept of pullback (or cocycle or non-autonomous) attractor provides a time-dependent (or random in the stochastic case) family of compact sets which attracts families of sets in a certain universe (e.g. the bounded sets in the phase space) and satisfying an invariance property, what seems to be a natural set of conditions to be satisfied for an appropriate extension of the autonomous concept of attractor. Moreover, this cocycle formulation allows to handle more general time-dependent terms in the models not only the periodic, quasi periodic or almost periodic ones (see, for instance, [Caraballo \& Real, 2004, Caraballo et al., 2004] for non-autonomous models containing hereditary characteristics). Readers can consult the monographs [Carvalho et al., 2010, Kloeden \& Rasmussen, 2010] for more references, examples and information.

\subsection{Motivation of the process and skew- product flow formalisms}

The formulation of an autonomous dynamical system as a group or semi-group of mappings depends on the fact that such systems depend only on the elapsed time since starting $t-t_{0}$ and not directly on the current time $t$ or starting time $t_{0}$ themselves. For a non-autonomous system, both the current time $t$ and starting time $t_{0}$ are important. The most natural generalization of a semi-group formalism to non-autonomous dynamical systems includes both $t$ and $t_{0}$ instead of only $t-t_{0}$. This leads to the two-parameter semi-group or process formalism of a non-autonomous dynamical system. An alternative method includes an autonomous dynamical system as a driving mechanism which is responsible for, e.g., the temporal change of the vector field of a non-autonomous dynamical system. This leads to the skew product flow formalism of a non- autonomous dynamical system.

\subsection{Processes}

Consider an initial value problem for a nonautonomous ordinary differential equation in $\mathbb{R}^{d}$,

$$
\dot{x}=f(t, x), \quad x\left(t_{0}\right)=x_{0} .
$$

In contrast to the autonomous case, the solutions may now depend separately on the actual time $t$ and the starting time $t_{0}$ rather than only on the elapsed time $t-t_{0}$ since starting. For example, the scalar initial value problem

$$
\dot{x}=-2 t x, \quad x\left(t_{0}\right)=x_{0},
$$

has the explicit solution

$$
x(t)=x\left(t, t_{0}, x_{0}\right)=x_{0} e^{-\left(t^{2}-t_{0}^{2}\right)},
$$

where $t^{2}-t_{0}^{2}=\left(t-t_{0}\right)^{2}+2\left(t-t_{0}\right) t_{0}$ cannot be expressed in terms of $t-t_{0}$ alone.

Assuming global existence and uniqueness of solutions in forward time, the solutions form a continuous mapping $\left(t, t_{0}, x_{0}\right) \mapsto x\left(t, t_{0}, x_{0}\right) \in \mathbb{R}^{d}$ for $t \geq t_{0}$ with $t, t_{0} \in \mathbb{R}$ and $x_{0} \in \mathbb{R}^{d}$, with the initial value and evolution properties

(i) $x\left(t_{0}, t_{0}, x_{0}\right)=x_{0}$ for all $t_{0} \in \mathbb{R}$ and $x_{0} \in \mathbb{R}^{d}$,

(ii) $x\left(t_{2}, t_{0}, x_{0}\right)=x\left(t_{2}, t_{1}, x\left(t_{1}, t_{0}, x_{0}\right)\right)$ for all $t_{0} \leq t_{1} \leq t_{2}$ and $x_{0} \in \mathbb{R}^{d}$.

The evolution property (ii) is a consequence of the causality principle that the solutions are determined uniquely by their initial values (for the given differential equation).

\section{2(A). Process formulation of non- autonomous systems}

Solution mappings of non-autonomous ordinary differential equations are one of the main motivations for the process formulation [Dafermos, 1971] (see also [Hale, 1988]) of an abstract non-autonomous system on a complete metric state space $(X, d)$ and time set $\mathbb{T}$, where $\mathbb{T}=\mathbb{R}$ for a continuous time process and $\mathbb{T}=\mathbb{Z}$ for a discrete time process.

Definition 5.1. A process is a continuous mapping $\left(t, t_{0}, x_{0}\right) \mapsto \phi\left(t, t_{0}, x_{0}\right) \in X$ for $t \geq t_{0}$ with $t, t_{0} \in \mathbb{T}$ and $x_{0} \in X$, with the initial value and evolution properties 
(i) $\phi\left(t_{0}, t_{0}, x_{0}\right)=x_{0}$ for all $t_{0} \in \mathbb{T}$ and $x_{0} \in X$,

(ii) $\phi\left(t_{2}, t_{0}, x_{0}\right)=\phi\left(t_{2}, t_{1}, \phi\left(t_{1}, t_{0}, x_{0}\right)\right)$ for all $t_{0} \leq t_{1} \leq t_{2}$ and $x_{0} \in X$.

A process is often called a 2-parameter semigroup on $X$ in contrast with the 1-parameter semigroup of an autonomous semi-dynamical system since it depends on both the initial time $t_{0}$ and the actual time $t$ rather than just the elapsed time $t-t_{0}$.

\section{2(B). Examples of processes}

The solution $x\left(t, t_{0}, x_{0}\right)$ of the non-autonomous differential equation (40) on $\mathbb{R}^{d}$ defines a continuous time process under the assumption of global existence and uniqueness of solutions. Indeed, this was the motivating example behind the definition of a process. Similarly, a non-autonomous difference equation generates a discrete time process.

Example 5.2. Let $f_{n}: \mathbb{R}^{d} \rightarrow \mathbb{R}^{d}$ be continuous mappings for $n \in \mathbb{Z}$. Then, the non-autonomous difference equation

$$
x_{n+1}=f_{n}\left(x_{n}\right)
$$

generates a discrete time process $\phi$ which is defined for all $x_{0} \in \mathbb{R}^{d}$ and $n, m \in \mathbb{Z}$ with $n \geq m+1$ by $\phi\left(m, m, x_{0}\right):=x_{0}$ and

$$
\phi\left(n, m, x_{0}\right):=f_{n-1} \circ \cdots \circ f_{m}\left(x_{0}\right) .
$$

In particular, note that $x_{0} \mapsto \phi\left(n, m, x_{0}\right)$ is continuous as the composition of finitely many continuous mappings.

There are processes which do not involve either differential or difference equations.

Example 5.3. Consider a non-homogeneous Markov chain on a finite state space $\{1, \ldots, N\}$ with $d \times d$ probability transition matrices

$$
P\left(t_{0}, t\right)=\left(p_{i, j}\left(t_{0}, t\right)\right)_{i, j=1, \ldots, d}
$$

for all $t_{0}, t \in \mathbb{T}$ with $t \geq t_{0}$. Such transition matrices satisfy $P\left(t_{0}, t_{0}\right)=\mathbb{1}$, the $d \times d$ identity matrix, for all $t_{0} \in \mathbb{T}$. They also satisfy the so-called ChapmanKolmogorov property

$$
P\left(t_{0}, s\right) P(s, t)=P\left(t_{0}, t\right) \text { for all } t_{0} \leq s \leq t .
$$

Let $\Sigma_{d}$ denote the subset of $\mathbb{R}^{d}$ consisting of the $N$-dimensional probability row vectors, i.e., $p=$ $\left(p_{1}, \cdots, p_{d}\right) \in \Sigma_{d}$ satisfies $\sum_{i=1}^{d} p_{i}=1$ with $0 \leq p_{i} \leq$ 1 for $i=1, \cdots, d$. If the states of the Markov chain at time $t_{0}$ satisfy the probability vector $p\left(t_{0}\right) \in \Sigma_{d}$, then they are distributed according to a probability vector $p(t)=p\left(t_{0}\right) P\left(t_{0}, t\right)$ at time $t \geq t_{0}$.

Thus, the mapping $\phi$ defined by $\phi\left(t, t_{0}, p_{0}\right):=$ $p_{0} P\left(t_{0}, t\right)$ is a process on the state space $\Sigma_{d}$, which is in fact linear in the initial state component $p_{0}$ and thus continuous in this variable. Continuity in the time variables is trivial in the discrete time case and requires the additional assumption of continuity of the transition matrices in both of their variables in the continuous time case. The two-parameter semi-group property follows from the ChapmanKolmogorov property.

Finally, all the examples in Section 4 admit non-autonomous variants which generate processes in the case of uniqueness of solutions.

\subsection{An interesting property of processes}

A process can be reformulated as an autonomous semi-dynamical system, which has some interesting implications.

The extended phase space will be denoted by $\mathcal{X}:=\mathbb{T} \times X$, and define a mapping $\pi: \mathbb{T}^{+} \times \mathcal{X} \rightarrow \mathcal{X}$ by

$$
\pi\left(t,\left(t_{0}, x_{0}\right)\right):=\left(t+t_{0}, \phi\left(t+t_{0}, t_{0}, x_{0}\right)\right)
$$

for all $\left(t,\left(t_{0}, x_{0}\right)\right) \in \mathbb{T}^{+} \times \mathcal{X}$. Note that the variable $t$ in $\pi\left(t,\left(t_{0}, x_{0}\right)\right)$ is the time which has elapsed since starting at time $t_{0}$, while the actual time is $t+t_{0}$.

Theorem 5.4. $\pi$ is an autonomous semidynamical system on $\mathcal{X}$.

Proof. It is obvious that $\pi$ is continuous in its variables and satisfies the initial condition

$$
\pi\left(0,\left(t_{0}, x_{0}\right)\right)=\left(t_{0}, \phi\left(t_{0}, t_{0}, x_{0}\right)\right)=\left(t_{0}, x_{0}\right) .
$$

It also satisfies the (1-parameter) semi-group property

$$
\pi\left(s+t,\left(t_{0}, x_{0}\right)\right)=\pi\left(s, \pi\left(t,\left(t_{0}, x_{0}\right)\right)\right), \forall s, t \in \mathbb{T}^{+},
$$


since, by the evolution property (ii) of the process,

$$
\begin{aligned}
& \pi\left(s+t,\left(t_{0}, x_{0}\right)\right) \\
& =\left(s+t+t_{0}, \phi\left(s+t+t_{0}, t_{0}, x_{0}\right)\right) \\
& =\left(s+t+t_{0}, \phi\left(s+t+t_{0}, t+t_{0}, \phi\left(t+t_{0}, t_{0}, x_{0}\right)\right)\right) \\
& =\pi\left(s,\left(t+t_{0}, \phi\left(t+t_{0}, t_{0}, x_{0}\right)\right)\right. \\
& =\pi\left(s, \pi\left(t,\left(t_{0}, x_{0}\right)\right)\right) .
\end{aligned}
$$

This finishes the proof of this theorem.

The autonomous semi-dynamical system $\pi$ on the extended state space $\mathcal{X}$ generated by a process $\phi$ on the state space $X$ has some unusual properties. In particular, $\pi$ has no nonempty $\omega$-limit sets and, indeed, no compact subset of $\mathcal{X}$ is $\pi$-invariant. This is a direct consequence of the fact that time is a component of the state.

This has significant implications and means that many concepts for autonomous systems need to be modified appropriately to be of any use in the non-autonomous context. For example, note that a $\pi$-invariant subset $\mathcal{A}$ of $\mathcal{X}$ has the form $\mathcal{A}=\bigcup_{t_{0} \in \mathbb{T}}\left(t_{0}, A_{t_{0}}\right)$, where $A_{t_{0}}$ is a nonempty subset of $X$ for each $t_{0} \in \mathbb{T}$. Then the invariance property $\pi(t, \mathcal{A})=\mathcal{A}$ for $t \in \mathbb{T}^{+}$is equivalent to

$\phi\left(t+t_{0}, t_{0}, A_{t_{0}}\right)=A_{t+t_{0}} \quad$ for all $t \in \mathbb{T}^{+}$and $t_{0} \in \mathbb{T}$.

This will be used later on to motivate the definition of $\phi$-invariant sets for a process $\phi$.

\subsection{Motivation of skew product flows}

To motivate the concept of a skew product flow, first a triangular system of ordinary differential equations is considered in which the uncoupled component can be considered as the driving force in the equation for the other component.

\section{4(A). Triangular autonomous differential equations}

Consider an autonomous system of ordinary differential equations of the form

$$
\frac{\mathrm{d} p}{\mathrm{~d} t}=f(p), \quad \frac{\mathrm{d} x}{\mathrm{~d} t}=g(p, x)
$$

where $p \in \mathbb{R}^{n}$ and $x \in \mathbb{R}^{m}$, i.e., with a triangular structure.
Assuming global existence and uniqueness of solutions forwards in time, the system of differential equations (42) generates an autonomous semidynamical system $\pi$ on $\mathbb{R}^{n+m}$ which we will write in component form as

$$
\pi\left(t, p_{0}, x_{0}\right)=\left(p\left(t, p_{0}\right), x\left(t, p_{0}, x_{0}\right)\right),
$$

with initial condition $\pi\left(0, p_{0}, x_{0}\right)=\left(p_{0}, x_{0}\right)$.

There are two important points to observe here. Firstly, the $p$-component of the system is an independent autonomous system in its own right, i.e., its solution mapping $p=p\left(t, p_{0}\right)$ generates an autonomous semi-dynamical system on $\mathbb{R}^{n}$ and amongst other properties satisfies the semi-group property

$$
p\left(s+t, p_{0}\right)=p\left(s, p\left(t, p_{0}\right)\right) \text { for all } s, t \geq 0 .
$$

Secondly, the semi-group property for $\pi$ on $\mathbb{R}^{n+m}$, i.e.,

$$
\pi\left(s+t, p_{0}, x_{0}\right)=\pi\left(s, \pi\left(t, p_{0}, x_{0}\right)\right),
$$

can be expanded out componentwise as

$$
\begin{aligned}
\pi\left(s+t, p_{0}, x_{0}\right) & =\left(p\left(s+t, p_{0}\right), x\left(s+t, p_{0}, x_{0}\right)\right) \\
& =\left(p\left(s, p\left(t, p_{0}\right)\right), x\left(s+t, p_{0}, x_{0}\right)\right),
\end{aligned}
$$

using (43), and

$$
\begin{gathered}
\pi\left(s, \pi\left(t, p_{0}\right)\right) \\
=\left(p\left(s, p\left(t, p_{0}\right)\right), x\left(s, p\left(t, p_{0}\right), x\left(t, p_{0}, x_{0}\right)\right)\right) .
\end{gathered}
$$

These are identical for all $s, t \geq 0$ and all $\left(p_{0}, x_{0}\right) \in$ $\mathbb{R}^{n+m}$. Equating for the second components gives

$$
x\left(s+t, p_{0}, x_{0}\right)=x\left(s, p\left(t, p_{0}\right), x\left(t, p_{0}, x_{0}\right)\right),
$$

for all $s, t \geq 0$, which is a generalization of the semigroup property and known as the cocycle property.

Given a solution $p=p\left(t, p_{0}\right)$ of the $p$ component of the triangular system (42), the $x$ component becomes a non-autonomous ordinary differential equation in the $x$ variable on $\mathbb{R}^{m}$ of the form

$$
\frac{\mathrm{d} x}{\mathrm{~d} t}=g\left(p\left(t, p_{0}\right), x\right) \quad \text { where } t \geq 0 \text { and } x \in \mathbb{R}^{n} .
$$

Here $p=p\left(t, p_{0}\right)$ can be considered as "driving" the non-autonomous system here, i.e., as being responsible for the changes in the vector field with the passage of time. 
The solution $x(t)=x\left(t, p_{0}, x_{0}\right)$ with initial value $x(0)=x_{0}$ (which also depends on the choice of $p_{0}$ as a parameter through the driving solution $\left.p\left(t, p_{0}\right)\right)$ then satisfies the following.

(i) Initial condition:

$$
x\left(0, p_{0}, x_{0}\right)=x_{0} .
$$

(ii) Cocycle property:

$$
x\left(s+t, p_{0}, x_{0}\right)=x\left(s, p\left(t, p_{0}\right), x\left(t, p_{0}, x_{0}\right)\right) .
$$

(iii) Continuity condition:

$$
\left(t, p_{0}, x_{0}\right) \mapsto x\left(t, p_{0}, x_{0}\right) \text { is continuous. }
$$

The mapping $x: \mathbb{R}^{+} \times \mathbb{R}^{n} \times \mathbb{R}^{m} \rightarrow \mathbb{R}^{m}$ is called a cocycle mapping. It describes the evolution of the solution of the non-autonomous differential equation (45) with respect to the driving system. Note that the variable $t$ here is the time since starting at the state $x_{0}$ with the driving system at state $p_{0}$.

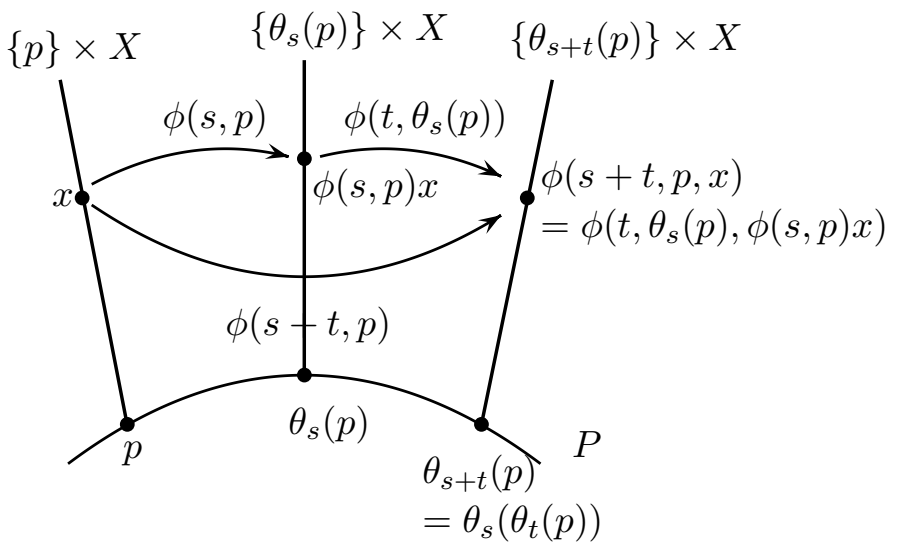

Fig. 1. The cocycle property

As mentioned above, the product system $\pi$ on $\mathbb{R}^{n} \times \mathbb{R}^{m}$ is an autonomous semi-dynamical system and is known as a skew product flow due to the asymmetrical roles of the two component systems. This motivates an alternative definition of a non-autonomous dynamical system, called the skew product flow formalism, where, for various reasons, the driving system $p$ is usually taken to be a reversible dynamical system, i.e., forming a group rather than a semi-group. This will happen for example, if the driving differential equation is restricted to a compact invariant subset of $\mathbb{R}^{n}$.

The process formulation of a non-autonomous dynamical system defined by the solution mapping of a non-autonomous differential equation is quite intuitive. In contrast, the skew product flow formulation is more abstract, but it contains more information about how the system evolves in time, especially when the driving system is on a compact space $P$ and in the case of a random perturbations, when $P$ is a probability space.

\subsection{Skew product flows}

Let $\left(X, d_{X}\right)$ and $\left(P, d_{P}\right)$ be metric spaces. A nonautonomous dynamical system $(\theta, \varphi)$ is defined in terms of a cocycle mapping $\varphi$ on a state space $X$ which is driven by an autonomous dynamical system $\theta$ acting on a base or parameter space $P$ and the time set $\mathbb{T}=\mathbb{R}$ or $\mathbb{Z}$.

Specifically, the dynamical system $\theta$ on $P$ is a group of homeomorphisms $\left(\theta_{t}\right)_{t \in \mathbb{T}}$ under composition on $P$ with the properties that

(i) $\theta_{0}(p)=p$ for all $p \in P$,

(ii) $\theta_{s+t}=\theta_{s}\left(\theta_{t}(p)\right)$ for all $s, t \in \mathbb{T}$,

(iii) the mapping $(t, p) \mapsto \theta_{t}(p)$ is continuous,

and the cocycle mapping $\varphi: \mathbb{T}^{+} \times P \times X \rightarrow X$ satisfies

(i) $\varphi(0, p, x)=x$ for all $(p, x) \in P \times X$,

(ii) $\varphi(s+t, p, x)=\varphi\left(s, \theta_{t}(p), \varphi(t, p, x)\right)$ for all $s, t \in \mathbb{T}^{+},(p, x) \in P \times X$,

(iii) the mapping $(t, p, x) \mapsto \varphi(t, p, x)$ is continuous.

The mapping $\pi: \mathbb{T}^{+} \times P \times X \rightarrow P \times X$ defined by

$$
\pi(t,(p, x)):=\left(\theta_{t}(p), \varphi(t, p, x)\right)
$$

forms an autonomous semi-dynamical system on $\mathcal{X}=P \times X$.

Definition 5.5. The autonomous semi-dynamical system $\pi$ on $\mathcal{X}=P \times X$ defined by (46) is called the skew product flow associated with the nonautonomous dynamical system $(\theta, \varphi)$.

\section{5(A). Examples of skew product flows}

Non-autonomous difference equations and differential equations provide a rich source of examples for skew product flows. 
Example 5.6. The solution mapping $x(t)=$ $x\left(t, t_{0}, x_{0}\right)$ of a non-autonomous differential equation (40) with initial value $x\left(t_{0}, t_{0}, x_{0}\right)=x_{0}$ at time $t_{0}$ defines a process. Theorem 5.4 shows that such a process can be reformulated as a skew product flow with the cocycle mapping $\varphi$ on the state space $X=\mathbb{R}^{d}$ defined by $\varphi\left(t, t_{0}, x_{0}\right):=x\left(t, t_{0}, x_{0}\right)$ and the driving system $\theta$ on the base space $P=\mathbb{R}$ defined by the shift operators $\theta_{t}\left(t_{0}\right):=t-t_{0}$. The disadvantages of this representation were discussed above.

The advantages of the skew product flow formulation reveals itself, for instance, when the generating non-autonomous differential equation is periodic or almost periodic in time, because the base space is then compact.

Example 5.7. The skew product formulation of a non-autonomous differential equation (40) is based on the fact that whenever $x(t)$ is a solution of the differential equation, then the time-shifted solution $x_{\tau}(t):=x(\tau+t)$ (for some fixed $\tau$ ) satisfies the non-autonomous differential equation

$$
\dot{x}_{\tau}(t)=f_{\tau}\left(t, x_{\tau}(t)\right):=f(\tau+t, x(\tau+t)) .
$$

Consider the set of functions $\left\{f_{\tau}(\cdot, \cdot):=f(\tau+\cdot, \cdot)\right.$ : $\tau \in \mathbb{R}\}$. Its closure $\mathcal{F}$ in an appropriate topology is called the hull of the vector field in the non-autonomous differential equation (40). See [Sell, 1971] for examples and typical topologies. For example, $\mathcal{F}$ is a compact metric space for periodic or almost periodic differential equations.

Introduce a group of shift operators $\theta_{\tau}: \mathcal{F} \mapsto \mathcal{F}$ by $\theta_{\tau}(f):=f_{\tau}$ for each $\tau \in \mathbb{R}$, define $\mathcal{X}=\mathcal{F} \times \mathbb{R}^{d}$ and write $\varphi\left(t, f, x_{0}\right)$ for the solution of (40) with initial value $x_{0}$ at initial time $t_{0}=0$. Finally, define $\pi$ : $\mathbb{R}^{+} \times \mathcal{X} \rightarrow \mathcal{X}$ by $\pi\left(t, x_{0}, f\right):=\left(\theta_{t}(f), \varphi\left(t, f, x_{0}\right)\right)$.

Then, $\pi=(\theta, \varphi)$ is a continuous-time skew product flow on the state space $\mathcal{X}$. To see this, observe that the second component of the semi-group identity $\pi\left(t+s, f, x_{0}\right)=\pi\left(t, \pi\left(s, f, x_{0}\right)\right.$ expands out as the cocycle property

$$
\varphi\left(t+s, f, x_{0}\right)=\varphi\left(t, \theta_{s}(f), \varphi\left(s, f, x_{0}\right)\right) .
$$

Non-autonomous difference equations (41) generate discrete time skew product flows, the simplest coming from discrete time processes via Theorem 5.4 and have $\mathbb{Z}$ as their base space. When more is known about how the different mappings $f_{n}$ vary with $n \in \mathbb{Z}$, it is often possible to have a compact base space.

Example 5.8. Suppose that the mappings $f_{n}$ in the non-autonomous difference equation (41) are chosen in some way from a finite number of continuous mappings $R_{i}: \mathbb{R}^{d} \rightarrow \mathbb{R}^{d}$ for $i \in\{1, \ldots, r\}$. Then the difference equation has the form

$$
x_{n+1}=R_{i_{n}}\left(x_{n}\right),
$$

where the $i_{n} \in\{1, \ldots, r\}$ for all $n \in \mathbb{N}$. It generates a discrete time skew product flow over the parameter set $P=\{1, \ldots, r\}^{\mathbb{Z}}$ of bi-infinite sequences $p=\left(i_{n}\right)_{n \in \mathbb{Z}}$ in $\{1, \ldots, r\}$ with respect to the group of left shift operators $\left(\theta_{m}\right)_{m \in \mathbb{N}}$, where $\theta_{m}\left(\left(i_{n}\right)_{n \in \mathbb{Z}}\right)=\left(i_{n+m}\right)_{n \in \mathbb{Z}}$. The cocycle mapping $\varphi(n, \cdot, \cdot)$ is defined by

$$
\varphi(0, p, x):=x, \varphi(n, p, x):=\left(R_{i_{n-1}} \circ \cdots \circ R_{i_{0}}\right)(x)
$$

for all $n \in \mathbb{N}, x \in \mathbb{R}^{d}$ and $p=\left(i_{n}\right)_{n \in \mathbb{N}} \in P$. The parameter space $P=\{1, \ldots, r\}^{\mathbb{Z}}$ here is a compact metric space with the metric

$$
d\left(p, p^{\prime}\right)=\sum_{n=-\infty}^{\infty}(r+1)^{-|n|}\left|i_{n}-i_{n}^{\prime}\right|,
$$

and the mappings $p \mapsto \theta_{n}(p)$ and $(p, x) \mapsto \varphi(n, p, x)$ are continuous for each $n \in \mathbb{Z}^{+}$. To see this, note that $d\left(p, p^{\prime}\right) \leq \delta<1$ requires $i_{j}=i_{j}^{\prime}$ for $j=0, \pm 1, \ldots, \pm N(\delta)$. Then take $\delta$ small enough corresponding to a given $\varepsilon>0$ and fixed $n$.

More generally, the difference equation may involve a parameter $q \in Q$, which varies from iterate to iterate, by choice or randomly,

$$
x_{n+1}=f\left(x_{n}, q_{n}\right) .
$$

Example 5.9. Consider the parametrically dependent difference equation (48) with the continuous mapping $f: \mathbb{R} \times[-1,1] \rightarrow \mathbb{R}$, given by

$$
f(x, q)=f_{q}(x):=\nu x+q,
$$

where $\nu \in[0,1)$ and $q \in[-1,1]$. Let $P=[-1,1]^{\mathbb{Z}}$ be the space of bi-infinite sequences $p=\left(q_{n}\right)_{n \in \mathbb{Z}}$ taking values in $[-1,1]$, which is a compact metric space with the metric

$$
d\left(p, p^{\prime}\right)=\sum_{n=-\infty}^{\infty} 2^{-|n|}\left|q_{n}-q_{n}^{\prime}\right|,
$$


and let $\left(\theta_{n}\right)_{n \in \mathbb{Z}}$ be the group of the left shift operators on this sequence space (cf. Example 5.8). Finally, define the mappings $\varphi(n, \cdot, \cdot)$ by

$$
\varphi\left(0, p, x_{0}\right):=x, \varphi(n, p, x):=\left(f_{q_{n-1}} \circ \cdots \circ f_{q_{0}}\right)(x)
$$

for all $n \in \mathbb{N}, x \in \mathbb{R}$ and $p=\left(q_{n}\right)_{n \in \mathbb{N}} \in P$. Specifically,

$$
\varphi(n, p, x)=\nu^{n} x+\sum_{j=0}^{n-1} \nu^{n-1-j} q_{j}
$$

for all $n \in \mathbb{N}$.

The mappings $p \mapsto \theta_{n}(p)$ and $(p, x) \mapsto$ $\varphi(n, p, x)$ are obviously continuous here for each $n \in \mathbb{N}$. Thus, $(\theta, \varphi)$ is a discrete time skew product flow on $\mathbb{R}$ with the compact base space $P$.

\section{5(B). Entire solutions and invariant sets}

The definition of an entire solution of a nonautonomous dynamical system is an obvious generalization of the autonomous case.

Definition 5.10. An entire solution of a process $\phi$ on a metric space $(X, d)$ with time set $\mathbb{T}$ is a mapping $\xi: \mathbb{T} \rightarrow X$ such that

$$
\xi(t)=\phi\left(t, t_{0}, \xi\left(t_{0}\right)\right) \quad \text { for all } t, t_{0} \in \mathbb{T} \text { with } t \geq t_{0} \text {. }
$$

The discussion following Theorem 5.4, in which a process $\phi$ on $X$ was formulated as a skew product flow on the extended state space $\mathbb{R} \times X$, suggests that it is more appropriate to consider the invariance of a family of time-dependent subsets rather than of a single set. This motivates the following definition.

Definition 5.11. Let $\phi$ be a process on a metric space $(X, d)$. A family $\mathcal{A}=\left(A_{t}\right)_{t \in \mathbb{T}}$ of nonempty subsets of $X$ is said to be invariant with respect to $\phi$, or $\phi$-invariant, if

$$
\phi\left(t, t_{0}, A_{t_{0}}\right)=A_{t} \quad \text { for all } t \geq t_{0} .
$$

A simple example of a $\phi$-invariant family $\mathcal{A}=$ $\left(A_{t}\right)_{t \in \mathbb{T}}$ is given by an entire solution of $\phi$, i.e., having the singleton subsets $A_{t}=\{\xi(t)\}$ for each $t \in \mathbb{T}$. In fact, $\phi$-invariant families consist of entire solutions.

Lemma 5.12. Let $\mathcal{A}=\left(A_{t}\right)_{t \in \mathbb{T}}$ be a nonempty $\phi$ invariant family of subsets of $X$ of a process $\phi$.
Then for any $t_{0} \in \mathbb{T}$ and $a_{0} \in A_{t_{0}}$, there exists an entire solution $\xi$ through $a_{0}$ which is contained in $\mathcal{A}$, i.e., with $\xi\left(t_{0}\right)=a_{0}$ and $\xi(t) \in A_{t}$ for all $t \in \mathbb{T}$.

When the subsets in a $\phi$-invariant family are compact, it follows from the continuity of a continuous time process that the set-valued mapping $t \mapsto A_{t}$ is continuous in $t \in \mathbb{R}$ with respect to the Hausdorff metric $h$, since

$$
h\left(A_{t}, A_{t_{0}}\right)=h\left(\phi\left(t, t_{0}, A_{t_{0}}\right), \phi\left(t_{0}, t_{0}, A_{t_{0}}\right)\right) \rightarrow 0
$$

as $t \rightarrow t_{0}$ by the continuity of the process $\phi$ in its first variable.

Similar definitions hold for positive and negative invariant families of sets.

Definition 5.13. Let $\phi$ be a process on a metric space $(X, d)$. A family $A=\left(A_{t}\right)_{t \in \mathbb{T}}$ of nonempty subsets of $X$ is said to be positive invariant with respect to $\phi$, or $\phi$-positive invariant, if

$$
\phi\left(t, t_{0}, A_{t_{0}}\right) \subset A_{t} \quad \text { for all } t \geq t_{0},
$$

and negative invariant with respect to $\phi$, or $\phi$ negative invariant, if this holds with $\supset$ instead of c.

Analogous definitions hold for skew product flows.

Definition 5.14. An entire solution of a skew product flow $(\theta, \varphi)$ on a metric phase space $(X, d)$ and a base set $P$ with time set $\mathbb{T}$ is a mapping $\xi: P \rightarrow X$ such that

$$
\xi\left(\theta_{t}(p)\right)=\varphi\left(t-s, \theta_{s}(p), \xi\left(\theta_{s}(p)\right)\right.
$$

for all $p \in P$ and $s, t \in \mathbb{T}$ with $s \leq t$.

Definition 5.15. Let $(\theta, \varphi)$ be a skew product flow on a metric phase space $(X, d)$ and a base set $P$. A family $A=\left(A_{p}\right)_{p \in P}$ of nonempty sets of $X$ is said to be invariant with respect to $(\theta, \varphi)$, or $\varphi$-invariant, if

$$
\varphi\left(t, p, A_{p}\right)=A_{\theta_{t}(p)} \quad \text { for all } t \geq 0 \text { and } p \in P .
$$

For positive and negative invariant families, replace $=$ here by $\subset$ or $\supset$, respectively. 
The compact set-valued mapping $t \mapsto A_{\theta_{t}(p)}$ induced by a $\varphi$-invariant family $\left(A_{p}\right)_{p \in P}$ of compact subsets is continuous in $t \in \mathbb{R}$ with respect to the Hausdorff metric for each fixed $p \in P$.

\subsection{Attractors for non-autonomous and random dynamical systems}

Simple generalizations of concepts for autonomous dynamical systems to non-autonomous dynamical systems are not always adequate or appropriate. It was previously seen that, for non-autonomous dynamical systems, it is often too restrictive to consider the invariance of just a single set and that instead a family of subsets is more appropriate. A similar situation also applies to attractors, which are the most important examples of invariant sets.

Attractors of autonomous dynamical systems are $\omega$-limit sets, which are invariant sets. Since the solution of a process $\varphi$ depends both on initial time $t_{0}$ and initial value $x_{0}, \omega$-limit set for a process will also depend on both of these two parameters, specifically

$$
\begin{gathered}
\omega\left(t_{0}, x_{0}\right)=\left\{x \in X: \lim _{n \rightarrow \infty} \varphi\left(t_{n}, t_{0}, x_{0}\right)=x\right. \\
\text { for some sequence } \left.t_{n} \rightarrow \infty\right\} .
\end{gathered}
$$

As in the autonomous case, one can show that $\omega\left(t_{0}, x_{0}\right)$ is a nonempty compact set when, for example, the forward trajectory $\cup_{t \geq t_{0}}\left\{\varphi\left(t, t_{0}, x_{0}\right)\right\}$ is precompact. However, unlike its autonomous counterpart, a non-autonomous $\omega$-limit set $\omega\left(t_{0}, x_{0}\right)$ may not be invariant for the process.

As an example, consider the non-autonomous scalar differential equation

$$
\dot{x}=-x+e^{-t},
$$

which can be solved with the variation of constants formula to give the explicit solution

$$
x\left(t, t_{0}, x_{0}\right)=e^{-\left(t-t_{0}\right)} x_{0}+\left(t-t_{0}\right) e^{-t} .
$$

This implies that

$$
\lim _{t \rightarrow \infty} x\left(t, t_{0}, x_{0}\right)=0 \quad \text { for all }\left(t_{0}, x_{0}\right) \in \mathbb{R} \times \mathbb{R},
$$

so the non-autonomous $\omega$-limit set is given by

$$
\omega\left(t_{0}, x_{0}\right)=\{0\} \quad \text { for all }\left(t_{0}, x_{0}\right) \in \mathbb{R} \times \mathbb{R} .
$$

However, $x\left(t, t_{0}, 0\right)=\left(t-t_{0}\right) e^{-t} \neq 0$ for all $t>t_{0}$, i.e., the $\omega$-limit set here is not invariant in the sense of autonomous systems.

\section{6(A). Non-autonomous sets}

Let $\phi$ be a process on a metric space $(X, d)$, and consider a family $\tilde{\mathcal{M}}=\left(M_{t}\right)_{t \in \mathbb{T}}$ of subsets of $X$. For a more compact and elegant formulation, such families $\tilde{\mathcal{M}}$ will henceforth be viewed equivalently as subsets of the extended phase space $\mathbb{T} \times X$, and the translation is as follows. The family $\tilde{\mathcal{M}}$ induces a subset $\mathcal{M} \subset \mathbb{T} \times X$, defined by

$$
\mathcal{M}:=\left\{(t, x): x \in M_{t}\right\}
$$

and, conversely, a subset $\mathcal{M}$ of the extended phase space $\mathbb{T} \times X$ leads to a family $\tilde{\mathcal{M}}=\left(M_{t}\right)_{t \in \mathbb{T}}$ of subsets of $X$ with

$$
M_{t}:=\{x \in X:(t, x) \in \mathcal{M}\} \quad \text { for all } t \in \mathbb{T} .
$$

The advantage of the new formulation is that $\mathcal{M}$ is a set, which makes the direct use of set-valued operations possible, and the notation becomes easier to read. Such sets $\mathcal{M}$ are called non-autonomous sets in the following. It will become clear soon that all interesting objects in the non-autonomous context are non-autonomous sets, i.e., subsets of the extended phase space, whereas the main interest focusses on subsets of the phase space in an autonomous setting. The precise definition of a nonautonomous set is:

Definition 5.16. Let $\phi$ be a process on a metric space $(X, d)$. A subset $\mathcal{M}$ of the extended phase space $\mathbb{T} \times X$ is called a non-autonomous set and for each $t \in \mathbb{T}$ the set

$$
M_{t}:=\{x \in X:(t, x) \in \mathcal{M}\}
$$

is called the $t$-fiber of $\mathcal{M}$. A non-autonomous set $\mathcal{M}$ is said to be invariant if $\phi\left(t, t_{0}, M_{t_{0}}\right)=M_{t}$ for all $t \geq t_{0}$.

An analogous notion of a non-autonomous set is also used for skew product flows.

Definition 5.17. Let $(\theta, \varphi)$ be a skew product flow on a base set $P$ and a metric phase space $(X, d)$. A subset $\mathcal{M}$ of the extended phase space $P \times X$ is called a non-autonomous set and for each $p \in P$ the set

$$
M_{p}:=\{x \in X:(p, x) \in \mathcal{M}\}
$$

is called $p$-fiber of $\mathcal{M}$. A non-autonomous set $\mathcal{M}$ is said to be invariant if $\varphi\left(t, p, M_{p}\right)=M_{\theta_{t}(p)}$ for all $t \geq 0$ and $p \in P$. 
In general, a non-autonomous set $\mathcal{M}$ is said to have a topological property (such as compactness or closedness) if each fiber of $\mathcal{M}$ has this property.

\section{6(B). Attractors of processes}

There are basically two ways to define attraction of a compact and invariant non-autonomous set $\mathcal{A}$ for a process $\phi$ on a metric space $(X, d)$ with time set $\mathbb{T}$. The first, and perhaps more obvious, corresponds to the attraction in Lyapunov asymptotic stability, is called forward attraction and involves a moving target, while the latter, called pullback attraction, involves a fixed target set with progressively earlier starting time. In general, these two types of attraction are independent concepts, while for the autonomous case, they are equivalent.

Definition 5.18. Let $\phi$ be a process. A nonempty, compact and invariant non-autonomous set $\mathcal{A}$ is said to be

(i) forward attracting if

$$
\lim _{t \rightarrow \infty} \operatorname{dist}\left(\phi\left(t, t_{0}, x_{0}\right), A_{t}\right)=0
$$

for all $x_{0} \in X$ and $t_{0} \in \mathbb{T}$,

(ii) and pullback attracting if

$$
\lim _{t_{0} \rightarrow-\infty} \operatorname{dist}\left(\phi\left(t, t_{0}, x_{0}\right), A_{t}\right)=0
$$

for all $x_{0} \in X$ and $t \in \mathbb{T}$.

Moreover, if the forward attraction in (i) is uniform with respect to $t_{0} \in \mathbb{T}$, or equivalently, if the pullback attraction in (ii) is uniform with respect to $t \in \mathbb{T}$, then $\mathcal{A}$ is called uniformly attracting.

Figures 5.6(B) and 5.6(B) illustrate forward and pullback attraction, respectively, of a nonautonomous set with singleton sets as fibers $A_{t}=$ $\{\rho(t)\}$, i.e., an entire solution of the process.

In an autonomous system, the solutions depend only on the elapsed time $t-t_{0}$. Moreover, $t-t_{0} \rightarrow$ $\infty$ when either $t \rightarrow \infty$ with $t_{0}$ fixed or as $t_{0} \rightarrow-\infty$ with $t$ fixed, so pullback and forward convergence are equivalent for an autonomous system.

Two types of non-autonomous attractors for processes are possible, depending which of the above types of attraction is used. It is required that the component subsets of such attractors are

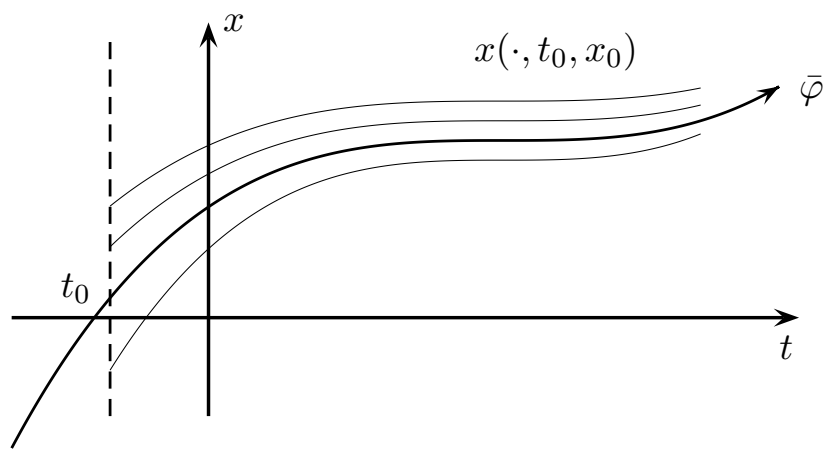

Fig. 2. Forward attraction

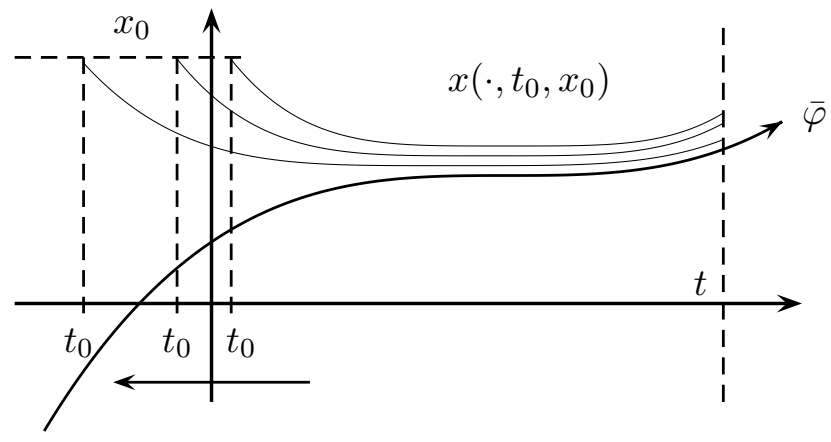

Fig. 3. Pullback Attraction

compact and that they attract bounded subsets $D$ of initial values in $X$ (rather than just individual points), in the sense that

$$
\operatorname{dist}\left(\phi\left(t, t_{0}, D\right), A_{t}\right) \rightarrow 0,
$$

either as $t \rightarrow \infty$ with $t_{0}$ fixed (forward case), or as $t_{0} \rightarrow-\infty$ with $t$ fixed (pullback case).

Definition 5.19. Let $\phi$ be a process. A nonempty and invariant non-autonomous set $\mathcal{A}$ is called

(i) a forward attractor if it forward attracts bounded subsets of $X$,

(ii) a pullback attractor if it pullback attracts bounded subsets of $X$, and

(iii) a uniform attractor if it uniformly attracts bounded subsets of $X$.

Forward and pullback attractors are independent concepts and one can exist without the other.

Example 5.20. The non-autonomous set $\mathbb{T} \times\{0\}$, i.e., the zero solution, is a forward attractor but 
not a pullback attractor of the system

$$
\dot{x}=-2 t x
$$

with the general solution $x\left(t, t_{0}, x_{0}\right)=x_{0} e^{-\left(t^{2}-t_{0}^{2}\right)}$, and a pullback attractor but not a forward attractor of the system

$$
\dot{x}=2 t x
$$

with the general solution $x\left(t, t_{0}, x_{0}\right)=x_{0} e^{t^{2}-t_{0}^{2}}$.

This example demonstrates that forward attraction can be seen as an attraction concept for the future of the system, since the coefficient $-2 t$ of (50) is negative for $t>0$. On the other hand, pullback attraction means basically attraction for the past of the system, see the negativity of $2 t$ for $t<0$ in (51). The concept of a uniform attractor, however, is concerned with attractivity for the entire time.

Example 5.21. Consider the non-autonomous scalar ordinary differential equation

$$
\dot{x}=-x+2 \sin t .
$$

If $x_{1}(t)$ and $x_{2}(t)$ are any two solutions, then their difference $z(t)=x_{1}(t)-x_{2}(t)$ satisfies the homogeneous linear differential equation

$$
\dot{z}=-z
$$

with the explicit solution $z(t)=z\left(t_{0}\right) e^{-\left(t-t_{0}\right)}$, so

$$
\left|x_{1}(t)-x_{2}(t)\right|=\left|x_{1}\left(t_{0}\right)-x_{2}\left(t_{0}\right)\right| e^{-\left(t-t_{0}\right)} \rightarrow 0
$$

as $t \rightarrow \infty$, from which it follows that all solutions converge to each other in time. What do they converge to?

The explicit solution of the non-autonomous differential equation (52) with initial value $x\left(t_{0}\right)=$ $x_{0}$ is

$$
\begin{aligned}
x\left(t, t_{0}, x_{0}\right)= & x_{0} e^{-\left(t-t_{0}\right)}+2 e^{-t} \int_{t_{0}}^{t} e^{s} \sin s \mathrm{~d} s \\
= & \left(x_{0}-\left(\sin t_{0}-\cos t_{0}\right)\right) e^{-\left(t-t_{0}\right)} \\
& +(\sin t-\cos t),
\end{aligned}
$$

from which it is clear that the forward limit $\lim _{t \rightarrow \infty} x\left(t, t_{0}, x_{0}\right)$ does not exist. On the other hand, the pullback limit does exist for all $t$ and $x_{0}$, i.e.,

$$
\lim _{t_{0} \rightarrow-\infty} x\left(t, t_{0}, x_{0}\right)=\sin t-\cos t=: \rho(t),
$$

and is independent of $x_{0}$, i.e.,

$$
\lim _{t_{0} \rightarrow-\infty}\left|x\left(t, t_{0}, x_{0}\right)-\rho(t)\right|=0 .
$$

Hence, the non-autonomous set $\mathcal{A}$ having the singleton fibers $A_{t}:=\{\rho(t)\}$ is pullback attracting for the solution process.

Moreover, it is easily shown that $\rho(t)$ is a solution of the non-autonomous differential equation (52) and since all solutions converge to each other forward in time, the forward convergence

$$
\lim _{t \rightarrow \infty}\left|x\left(t, t_{0}, x_{0}\right)-\rho(t)\right|=0
$$

also holds.

Remark 5.22. The non-autonomous set $\mathcal{A}$ in Example 5.21 is both a pullback and forward attractor of the non-autonomous differential equation (52). It is not difficult to find other forward attractors of (52) which are not pullback attractors.

This fact demonstrates that forward attractors can be nonunique, and this is quite typical for forward attractors. For pullback attractors, however, the following uniqueness result can be proved.

Proposition 5.23. Suppose that a process $\phi$ has two pullback attractors $\mathcal{A}$ and $\overline{\mathcal{A}}$ such that both $\bigcup_{t \leq 0} A_{t}$ and $\bigcup_{t \leq 0} \bar{A}_{t}$ are bounded. Then $\mathcal{A}=\overline{\mathcal{A}}$.

Proof. The boundedness of $\bigcup_{t \leq 0} A_{t}$ implies for all $t \in \mathbb{T}$ that

$$
\begin{aligned}
\operatorname{dist}\left(A_{t}, \bar{A}_{t}\right) & =\lim _{t_{0} \rightarrow-\infty} \operatorname{dist}\left(\phi\left(t, t_{0}, A_{t_{0}}\right), \bar{A}_{t}\right) \\
& \leq \lim _{t_{0} \rightarrow-\infty} \operatorname{dist}\left(\phi\left(t, t_{0}, \bigcup_{\tau \leq 0} A_{\tau}\right), \bar{A}_{t}\right)=0 .
\end{aligned}
$$

Analogously, one shows that $\operatorname{dist}\left(\bar{A}_{t}, A_{t}\right)=0$, which finishes the proof, since both the sets $A_{t}$ and $\bar{A}_{t}$ are compact.

Exercise 5.24. A $\phi$-invariant family of sets such as a pullback attractor $A=\left(A_{t}\right)_{t \in \mathbb{T}}$ consists of entire solutions, see Lemma 5.12. Give an example of a process $\phi$ which has entire solutions that are not contained in the pullback attractor.

\subsection{Attractors of skew product flows}

Let $(\theta, \varphi)$ be a skew product flow on a base space $P$ and a state space $X$ with time set $\mathbb{T}=\mathbb{R}$ or $\mathbb{Z}$, where $\left(P, d_{P}\right)$ and $\left(X, d_{X}\right)$ are metric spaces. 
Then $\pi=(\theta, \varphi)$ is an autonomous semidynamical system on the extended state space $\mathcal{X}:=$ $P \times X$. A global attractor for an autonomous semidynamical system $\pi$ is, specifically, a nonempty compact subset $\mathcal{A}$ of $\mathcal{X}$ which is $\pi$-invariant, i.e., which satisfies $\pi(t, \mathcal{A})=\mathcal{A}$ for all $t \in \mathbb{T}^{+}$is called a global attractor of $\pi$ if

$$
\lim _{t \rightarrow \infty} \operatorname{dist}_{\mathcal{X}}(\pi(t, D), \mathcal{A})=0
$$

for every nonempty bounded subset $D$ of $\mathcal{X}$. Suppose that $P$ is compact and $\theta$-invariant. Then the global attractor $\mathcal{A}$ of $\pi$ has the form

$$
\mathcal{A}=\bigcup_{p \in P}\left\{(p, x): x \in A_{p}\right\},
$$

where $A_{p}$ is a nonempty compact subset of $X$ for each $p \in P$, and the $\pi$-invariance property $\pi(t, \mathcal{A})=$ $\mathcal{A}$ for $t \in \mathbb{T}^{+}$is equivalent to the $\varphi$-invariance property $\varphi\left(t, p, A_{p}\right)=A_{\theta_{t}(p)}$ for all $t \in \mathbb{T}^{+}$and $p \in P$.

A global attractor of the autonomous system $\pi$ is a possible candidate for an attractor for the nonautonomous dynamical system described by the skew product flow. A disadvantage of this definition is that the extended state space $\mathcal{X}$ includes the base space $P$ as a component, which often does not have the same physical significance as the state space $X$.

Other types of attractors consisting of a family of nonempty compact subsets of the state space $X$ have also been proposed for a skew product $(\theta, \varphi)$. These are analogues of the forward and pullback attractors of a process.

Definition 5.25. Let $(\theta, \varphi)$ be a skew product flow. A nonempty, compact and invariant nonautonomous set $\mathcal{A}$ is called a pullback attractor of $(\theta, \varphi)$ if the pullback convergence

$$
\lim _{t \rightarrow \infty} \operatorname{dist}_{X}\left(\varphi\left(t, \theta_{-t}(p), D\right), A_{p}\right)=0
$$

holds for every nonempty bounded subset $D$ of $X$ and $p \in P$, and is called a forward attractor if the forward convergence

$$
\lim _{t \rightarrow \infty} \operatorname{dist}_{X}\left(\varphi(t, p, D), A_{\theta_{t}(p)}\right)=0
$$

holds for every nonempty bounded subset $D$ of $X$ and $p \in P$.
As for processes, the concepts of forward and pullback attractors for skew products are generally independent of each other, and one can exist without the other existing. If the above limit is replaced by $\lim _{t \rightarrow \infty} \sup _{p \in P} \operatorname{dist}_{X}(\cdot, \cdot)$, then the attractors are called uniform pullback and uniform forward attractors, respectively. If either of the limits is uniform in this sense, then so is the other and the attractor is both a uniform pullback and a uniform forward attractor, so will be called simply a uniform attractor.

The relationship between these different kinds of non-autonomous attractors can be seen in [Cheban et al., 2002] and in the monograph [Kloeden \& Rasmussen, 2010].

Example 5.26. Reconsider the non-autonomous scalar ordinary differential equation (52), now writing $p(t)$, a periodic function with period $2 \pi$, instead of $\sin t$,

$$
\dot{x}=-x+2 p(t),
$$

with the initial condition $x(0)=x_{0}$. In the spirit of skew product flows as introduced in Section 5.4, the general solution of this equation depends on both $p$ and $x_{0}$. The initial value problem has thus the explicit solution $x(t)=x\left(t, p, x_{0}\right)$ given by

$$
x(t)=x_{0} e^{-t}+2 e^{-t} \int_{0}^{t} e^{s} p(s) \mathrm{d} s .
$$

We introduce shift operators on the space $P=$ $\{p(t+\cdot): 0 \leq t \leq 2 \pi\}$ defined by $\theta_{t}(p(\cdot))=p(t+\cdot)$ and consider the solution corresponding to the driving term $\theta_{-\tau}(p(\cdot))=p(-\tau+\cdot)$ at time $\tau$, i.e.,

$$
\begin{aligned}
x\left(\tau, \theta_{-\tau}(p(\cdot)), x_{0}\right) & =x_{0} e^{-\tau}+2 e^{-\tau} \int_{0}^{\tau} e^{s} \theta_{-\tau}(p(s)) \mathrm{d} s \\
& =x_{0} e^{-\tau}+2 e^{-\tau} \int_{0}^{\tau} e^{s} p(s-\tau) \mathrm{d} s \\
& =x_{0} e^{-\tau}+2 \int_{0}^{\tau} e^{s-\tau} p(s-\tau) \mathrm{d} s \\
& =x_{0} e^{-\tau}+2 \int_{-\tau}^{0} e^{t} p(t) \mathrm{d} t,
\end{aligned}
$$

where the substitution $t:=s-\tau$ has been used. The pullback limit as $\tau \rightarrow \infty$ gives

$$
\lim _{\tau \rightarrow \infty} x\left(\tau, \theta_{-\tau}(p(\cdot)), x_{0}\right)=\alpha(p(\cdot)):=2 \int_{-\infty}^{0} e^{t} p(t) \mathrm{d} t
$$


for $x_{0}$ in an arbitrary bounded subset $D$. It consists of singleton fibers $A_{p}=\{\alpha(p(\cdot))\}, p \in P$, and corresponds to the entire solution

$$
\rho(t):=\sin t-\cos t
$$

in the process version of this differential equation in Example 5.21, i.e., $\rho(t)=\alpha\left(\theta_{t}(p(\cdot))\right)$ for all $t \in \mathbb{R}$.

The pullback attraction in this example is uniform, and the pullback attractor is also a forward attractor, and hence a uniform attractor. Moreover, the autonomous semi-dynamical system $\pi=(\theta, \varphi)$ on the extended state space $P \times \mathbb{R}$ has a global attractor given by

$$
\mathcal{A}=\bigcup_{p(\cdot) \in P}\{(p(\cdot), \alpha(p(\cdot)))\} .
$$

Remark 5.27. The analysis of this system as a skew product flow is somewhat more complicated and less transparent than its analysis as a process in Example 5.21. This is typical and is why the process formulation will often be used in subsequent examples (whenever it is possible).

Example 5.28. The difference equation in Example 5.9 generates a discrete time skew product flow with cocycle mapping

$$
\varphi(n, p, x)=\nu^{n} x+\sum_{j=0}^{n-1} \nu^{n-1-j} q_{j} \quad \text { for all } n \in \mathbb{N},
$$

on the state space $X=\mathbb{R}$. The base space $P=$ $[-1,1]^{\mathbb{Z}}$ is the space of bi-infinite sequences $p=$ $\left(q_{n}\right)_{n \in \mathbb{N}}$ taking values in $[-1,1]$ and $\theta$ is the left shift operator on this sequence space.

Replacing $p$ by $\theta_{-n}(p)$ in (53) implies

$$
\varphi\left(n, \theta_{-n}(p), x\right)=\nu^{n} x+\sum_{j=0}^{n-1} \nu^{n-1-j} q_{-n+j} \forall n \in \mathbb{N},
$$

which can be re-indexed as

$$
\varphi\left(n, \theta_{-n}(p), x\right)=\nu^{n} x+\sum_{k=-n}^{-1} \nu^{-k-1} q_{k}, \forall n \in \mathbb{N} .
$$

Taking pullback convergence gives

$$
\lim _{n \rightarrow \infty} \varphi\left(n, \theta_{-n}(p), x\right)=\alpha(p):=\sum_{k=-\infty}^{-1} \nu^{-k-1} q_{k} .
$$

The pullback attractor $\mathcal{A}$ thus consists of singleton fibers $A_{p}=\{\alpha(p)\}$ for $p=\left(q_{n}\right)_{n \in \mathbb{N}} \in P$. Since the pullback convergence here is in fact uniform in $p \in$ $P$, the pullback attractor is also a uniform forward attractor, and hence a uniform attractor. Moreover, $\mathcal{A}$ is also the global attractor of the autonomous semi-dynamical system $\pi=(\theta, \varphi)$ on the extended state space $P \times \mathbb{R}$.

\section{7(A). Existence of pullback attractors}

In order to prove the existence of the attractor (in both the autonomous and non-autonomous cases) the simplest, and therefore the strongest, assumption is the compactness of the solution operator associated with the system, which is usually available for parabolic systems in bounded domain. However, this kind of compactness does not hold in general for parabolic equations in unbounded domains and hyperbolic equations on either bounded or unbounded domain. Instead we often have some kind of asymptotic compactness. In this situation, there are several approaches to prove the existence of the global (or non-autonomous) attractor. Roughly speaking, the first one ensures the existence of the global (resp. non-autonomous) attractor whenever a compact attracting set (resp. a family of compact attracting sets) exists. The second method consists in decomposing the solution operator (resp. the cocycle or two-parameter semi-group) into two parts: a compact part and another one which decays to zero as time goes to infinity. However, as it is not always easy to find this decomposition, one can use a third approach which is based on the use of the energy equations which are in direct connection with the concept of asymptotic compactness. This third method has been used fo example in [Moise et al., 1998] and [Lukaszewicz \& Sadowski, 2004] to extend to the non-autonomous situation the corresponding one in the autonomous framework (see [Rosa, 1998]), but related to uniform asymptotic compactness. Our aim here is to consider the case without uniformity properties and show how the pullback theory works in this situation. In this sense, it is worth mentioning that the compact case has been treated in [Flandoli \& Schmalfuß, 1996] in the random case, so our results can be considered as natural complements (as it happens in the deterministic autonomous case). 
There are generalizations of the theorems ensuring existence of an attractor for autonomous systems to pullback attractors for processes and skew product flows. These are also based on the supposed existence of an absorbing set, which is now absorbing in the pullback sense.

Definition 5.29. Let $\phi$ be a process on a metric space $(X, d)$. A nonempty compact subset $B$ of $X$ is called pullback absorbing if for each $t \in \mathbb{T}$ and every bounded subset $D$ of $X$, there exists a $T=T(t, D)>0$ such that

$$
\phi\left(t, t_{0}, D\right) \subset B \quad \text { for all } t_{0} \in \mathbb{T} \text { with } t_{0} \leq t-T \text {. }
$$

Definition 5.30. Let $(\theta, \phi)$ be a skew product flow on a metric space $(X, d)$. A nonempty compact subset $B$ of $X$ is called pullback absorbing if for each $p \in P$ and every bounded subset $D$ of $X$, there exists a $T=T(p, D)>0$ such that

$$
\varphi\left(t, \theta_{-t}(p), D\right) \subset B \text { for all } t \geq T .
$$

The existence theorems will be presented here under basic but restricted assumptions, which can then be relaxed and generalized.

\section{7(B). Existence of pullback attractors for pro- cesses}

The following theorem is a simple generalization of the corresponding one for attractors of autonomous semi-dynamical systems.

Theorem 5.31. Let $\phi$ be a process on a complete metric space $X$ with a compact pullback absorbing set $B$ such that

$$
\phi\left(t, t_{0}, B\right) \subset B \quad \text { for all } t \geq t_{0} .
$$

Then there exists a pullback attractor $\mathcal{A}$ with fibers in $B$ uniquely determined by

$$
A_{t}=\bigcap_{\tau \geq 0} \overline{\bigcup_{t_{0} \leq-\tau} \phi\left(t, t_{0}, B\right)} \quad \text { for all } t \in \mathbb{T} .
$$

The formula (54) is a kind of a non-autonomous $\omega$-limit set of the set $B$. As seen in the introduction of this chapter, a naive definition of a non-autonomous $\omega$-limit set leads to a set, which is not positively invariant. However, the pullback construction used in (54) gives an invariant set and can be regarded as a proper version of a non-autonomous $\omega$-limit set (see [Crauel \& Flandoli, 1994] for details).

Example 5.32. Consider a non-autonomous dynamical system in $\mathbb{R}^{d}$ given by

$$
\dot{x}=f(t, x),
$$

where $f$ is continuously differentiable and satisfies the uniform dissipative condition

$$
\langle x, f(t, x)\rangle \leq K-L\|x\|^{2}, \forall x \in \mathbb{R}^{d}, t \in \mathbb{R}
$$

with positive constants $K$ and $L$. These assumptions ensure that the differential equation (55) generates a process.

Moreover, any solution $x(t)$ of $(55)$ satisfies

$$
\begin{aligned}
\frac{\mathrm{d}}{\mathrm{d} t}\|x(t)\|^{2} & =2\langle x(t), \dot{x}(t)\rangle \\
& =2\langle x(t), f(t, x(t))\rangle \leq K-L\|x(t)\|^{2},
\end{aligned}
$$

from which, on integrating, it follows that

$$
\|x(t)\|^{2} \leq\left\|x\left(t_{0}\right)\right\|^{2} e^{-L\left(t-t_{0}\right)}+\frac{K}{L}\left(1-e^{-L\left(t-t_{0}\right)}\right) .
$$

Suppose that for a bounded subset $D$ of $\mathbb{R}^{d}$ with $\|D\|:=\sup _{d \in D}\|d\|>1$, we have $x\left(t_{0}\right) \in D$, and define

$$
T:=\frac{1}{L} \ln (L\|D\|) .
$$

Then

$$
\|x(t)\|^{2} \leq \frac{1}{L}+\frac{K}{L}=\frac{K+1}{L}
$$

for $x\left(t_{0}\right) \in D$ and $t_{0} \leq t-T$. Thus, the closed ball $B_{d}(0, \sqrt{(K+1) / L}):=\left\{x \in \mathbb{R}^{d}:\|x\|^{2} \leq(K+1) / L\right\}$ is pullback absorbing and positively invariant.

From Theorem 5.31, it follows that the process generated by the differential equation (55) has a pullback attractor in $\mathbb{R}^{d}$ with components subsets $A_{t} \subset B:=B_{d}(0, \sqrt{(K+1) / L})$.

\section{7(C). Existence of pullback attractors for skew product flows}

The counterpart of Theorem 5.31 for skew product flows is the first part of the following theorem. The second part provides some information about a form of forwards convergence of the cocycle mapping, which is different from that in the definition of a forward attractor. 
Theorem 5.33. Let $(\theta, \varphi)$ be skew product flow on a complete metric space $X$ with a compact pullback absorbing set $B$ such that

$$
\varphi(t, p, B) \subset B \quad \text { for all } t \geq 0 \text { and } p \in P .
$$

Then there exists a unique pullback attractor $\mathcal{A}$ with fibers in $B$ uniquely determined by

$$
A_{p}=\bigcap_{\tau \geq 0} \overline{\bigcup_{t \geq \tau} \varphi\left(t, \theta_{-t}(p), B\right)} \quad \text { for all } p \in P .
$$

If, in addition, $\left(P, d_{P}\right)$ is a compact metric space, then

$$
\lim _{t \rightarrow \infty} \sup _{p \in P} \operatorname{dist}(\varphi(t, p, D), A(P))=0
$$

for any bounded subset $D$ of $\mathbb{R}^{d}$, where $A(P):=$ $\overline{\bigcup_{p \in P} A_{p}} \subset B$.

\section{7(D). A continuous time example}

Consider a non-autonomous dynamical system in $\mathbb{R}^{d}$ given by

$$
\dot{x}=f(p, x)
$$

with the driving system $\theta$ on a compact metric space $\left(P, d_{P}\right)$. Suppose that $f$ is regular enough to ensure that the differential equation (60) generates a skew product flow.

In addition, suppose that $f$ satisfies the uniform dissipative condition

$$
\langle x, f(p, x)\rangle \leq K-L\|x\|^{2}, \forall p \in P, x \in \mathbb{R}^{d}
$$

with positive constants $K$ and $L$. Then, similarly to Example 5.32, a solution $x(t)$ satisfies the differential inequality

$$
\frac{\mathrm{d}}{\mathrm{d} t}\|x(t)\|^{2} \leq K-L\|x(t)\|^{2},
$$

from which it follows that the closed ball

$$
B:=B_{d}(0, \sqrt{(K+1) / L})
$$

is pullback absorbing and positively invariant. From Theorem 5.33, it follows that the skew product flow has a pullback attractor in $\mathbb{R}^{d}$ with components subsets $A_{p} \subset B, p \in P$.

Suppose instead that the vector field $f$ satisfies the uniform one-sided dissipative Lipschitz conditions

$$
\left\langle x_{1}-x_{2}, f\left(p, x_{1}\right)-f\left(p, x_{2}\right)\right\rangle \leq-L\left\|x_{1}-x_{2}\right\|^{2}
$$

for all $p \in P$ and $x_{1}, x_{2} \in \mathbb{R}^{d}$ with some constant $L>0$. Then $f$ satisfies the uniform dissipative condition (61) with constants

$$
K^{\prime}=\frac{2}{L} \sup _{p \in P}\|f(0)\| \quad \text { and } \quad L^{\prime}=\frac{L}{2},
$$

and the closed ball $B^{\prime}:=B_{d}\left(0, \sqrt{\left(K^{\prime}+1\right) / L^{\prime}}\right)$ is pullback absorbing and positively invariant. Thus, the skew product flow has a pullback attractor with component subsets $A_{p}$ in this ball.

In fact, the fibers of the pullback attractor are singleton sets. The proof uses the fact that due to the uniform one-sided dissipative Lipschitz condition (62), the system satisfies

$$
\left\|x_{1}(t)-x_{2}(t)\right\| \leq e^{-L t}\left\|x_{0,1}-x_{0,2}\right\|
$$

for any pair of solutions with the same initial value $p \in P$ of the driving system. This follows from

$$
\begin{aligned}
& \frac{\mathrm{d}}{\mathrm{d} t}\left\|x_{1}(t)-x_{2}(t)\right\|^{2} \\
& =\frac{\mathrm{d}}{\mathrm{d} t}\left\langle x_{1}(t)-x_{2}(t), x_{1}(t)-x_{2}(t)\right\rangle \\
& =2\left\langle x_{1}(t)-x_{2}(t), \dot{x}_{1}(t)-\dot{x}_{2}(t)\right\rangle \\
& =2\left\langle x_{1}(t)-x_{2}(t), f\left(\theta_{t} p, x_{1}(t)\right)-f\left(\theta_{t} p, x_{2}(t)\right)\right\rangle \\
& \leq-2 L\left\|x_{1}(t)-x_{2}(t)\right\|^{2},
\end{aligned}
$$

which is integrated to give

$$
\left\|x_{1}(t)-x_{2}(t)\right\|^{2} \leq e^{-2 L t}\left\|x_{0,1}-x_{0,2}\right\|^{2} .
$$

Taking square roots yields the desired result.

Theorem 5.34. The pullback attractor $\mathcal{A}$ of the skew product flow $(\theta, \varphi)$ generated by the differential equation (60) consists of singleton fibers $A_{p}=\left\{a_{p}\right\}$ for each $p \in P$ when the vector field $f$ satisfies the uniform one-sided dissipative Lipschitz condition (62). Moreover, $t \mapsto a_{\theta_{t}(p)}, t \in \mathbb{R}$, is an entire solution of (60) for each $p \in P$.

Proof. Since $A_{p} \subset B^{\prime}$ for all $p \in P$, it follows that $\left\|A_{p}\right\|:=\max _{a \in A_{p}}\|a\| \leq R:=\left(K^{\prime}+1\right) / L^{\prime}$ for each $p \in P$. Now consider a fixed $p \in P$, and suppose that there exists an $\varepsilon_{0}>0$ and points $a_{1}, a_{2} \in A_{p}$ such that $\left\|a_{1}-a_{2}\right\|=\varepsilon_{0}$. Moreover, choose $T>0$ such that $2 R e^{-L T}=\varepsilon_{0}$. The $\varphi$-invariance of the pullback attractor gives $\varphi\left(T, \theta_{-T}(p), A_{\theta_{-T}(p)}\right)=$ 
$A_{p}$, which means that there exist $a_{1}^{\prime}, a_{2}^{\prime} \in A_{\theta_{-T} p}$ such that

$\varphi\left(T, \theta_{-T}(p), a_{1}^{\prime}\right)=a_{1} \quad$ and $\quad \varphi\left(T, \theta_{-T}(p), a_{2}^{\prime}\right)=a_{2}$.

Then, from the inequality in (63), it follows that

$$
\begin{aligned}
0<\varepsilon_{0} & =\left\|a_{1}-a_{2}\right\| \\
& =\left\|\varphi\left(T, \theta_{-T}(p), a_{1}^{\prime}\right)-\varphi\left(T, \theta_{-T}(p), a_{2}^{\prime}\right)\right\| \\
& \leq e^{-L T}\left\|a_{1}^{\prime}-a_{2}^{\prime}\right\| \leq R e^{-L T}=\frac{1}{2} \varepsilon_{0},
\end{aligned}
$$

which is not possible. Hence, $a_{1}=a_{2}$.

Finally, from the $\varphi$-invariance of the pullback attractor, $\varphi\left(t, p, a_{p}\right)=a_{\theta_{t}(p)}$ for all $t \in \mathbb{R}$ and $p \in P$, so the singleton sets forming the pullback attractor define an entire solution of the system. It follows from inequality (63) that this entire solution also forward attracts all other solutions with the same initial value of the driving system, so the pullback attractor is also a forward attractor. (There will be more than one such entire solution when $P$ is not a minimal subset for the autonomous dynamical system $\theta$ ).

The above theorem generalizes the following autonomous result in [Stuart \& Humphries, 1996].

Corollary 5.35. An autonomous differential equation with a vector field $f$ which satisfies a one-sided dissipative Lipschitz condition such as (62) (i.e., without the $p$ variable) has a unique globally asymptotically stable equilibrium point.

\section{7(E). Pullback attractors for absorbing fami- lies and attraction universes}

To take into account non-uniformities that are ubiquitous in non-autonomous dynamical systems, greater generality can be attained in the definition of a pullback attractor by considering arbitrary non-autonomous sets $\mathcal{B}$ and $\mathcal{D}$ instead just a single compact absorbing set $B$ and single attracted bounded set $D$. This allows local as well as global attraction to be handled at the same time. The skew product flows $(\theta, \varphi)$ in this subsection are on a metric state space $\left(X, d_{X}\right)$ with a metric base space $\left(P, d_{P}\right)$ and a time set $\mathbb{T}$.

Definition 5.36. An attraction universe $\mathfrak{D}$ of a skew product flow $(\theta, \varphi)$ is a collection of bounded non-autonomous sets $\mathcal{D}$, which is closed in the sense that if $\emptyset \subsetneq \mathcal{D}^{\prime} \subseteq \mathcal{D}$ for some $\mathcal{D} \in \mathfrak{D}$, then $\mathcal{D}^{\prime} \in \mathfrak{D}$.
The definitions of pullback convergence and pullback attractor need to be extended accordingly.

Definition 5.37. Let $(\theta, \varphi)$ be a skew product flow on $P \times X$. A nonempty, compact and invariant nonautonomous set $\mathcal{A}$ is called pullback attractor with respect to an attraction universe $\mathfrak{D}$ if the pullback convergence

$$
\lim _{t \rightarrow \infty} \operatorname{dist}\left(\varphi\left(t, \theta_{-t}(p), D_{\theta_{-t}(p)}\right), A_{p}\right)=0
$$

holds for all $p \in P$ and $\mathcal{D} \in \mathfrak{D}$.

Exercise 5.38. Show that a pullback attractor is unique within a given attraction universe $\mathfrak{D}$.

The pullback absorbing property now depends on the attraction universe $\mathcal{D}$ under consideration.

Definition 5.39. Let $\mathfrak{D}$ be an attraction universe of a skew product flow $(\theta, \varphi)$ on $P \times X$. A nonempty and compact non-autonomous set $\mathcal{B} \in \mathfrak{D}$ is called pullback absorbing with respect to $\mathfrak{D}$ if for each $\mathcal{D} \in$ $\mathfrak{D}$ and $p \in P$, there exists a $T=T(p, D)>0$ such that

$$
\varphi\left(t, \theta_{-t}(p), D_{\theta_{-t}(p)}\right) \subset B_{p} \quad \text { for all } t \geq T .
$$

Theorem 5.33 on the existence of a pullback attractor assuming that of a pullback absorbing set generalizes to attraction universes and pullback absorbing families.

Theorem 5.40. Let $(\theta, \varphi)$ be a skew product flow on $P \times X$, and suppose that the compact nonautonomous set $\mathcal{B}$ is pullback absorbing with respect to an attraction universe $\mathfrak{D}$. Then $(\theta, \varphi)$ has a pullback attractor $\mathcal{A}$ with respect to $\mathfrak{D}$, where the fibers $A_{p}$ are defined for each $p \in P$ by

$$
A_{p}=\bigcap_{s>0} \overline{\bigcup_{t>s} \phi\left(t, \theta_{-t}(p), B_{\theta_{-t}(p)}\right)} .
$$

The proof is a direct modification of that of Theorem 5.33. Similar theorems can be found in [Schmalfuß, 1992, Flandoli \& Schmalfuß, 1996, Kloeden \& Schmalfuß, 1996, Kloeden, 1997], see also [Crauel et al., 1995, Cheban \& Fakhih, 1994].

Remark 5.41. The assumption that the absorbing sets in Theorems 5.33 and 5.40 are compact is no 
restriction in a state space such as $\mathbb{R}^{d}$, which is locally compact and thus closed and bounded subsets are equivalently compact. This is not true for a general state space. In particular, for infinite dimensional spaces, compact subsets are "thin" and it is much easier to determine an absorbing property for a closed and bounded subset, such as a unit ball, rather than a compact subset. Counterparts of Theorems 5.33 and 5.40 then hold, if the cocycle mapping is assumed to be compact, i.e., the mapping $\varphi(t, p, \cdot): X \rightarrow X$ maps bounded subsets into precompact subsets for all $t>0$ and $p \in P$, or more generally, asymptotically compact. These generalizations will be considered in the next section on infinite dimensional dynamical systems, i.e., with an infinite dimensional state space $X$, and dealing with possibly multi-valued non-autonomous and random dynamical systems. It is also remarkable that, when the problem contains some randomness or stochastic features, the parameter space possesses a measurable structure (i.e., is a probability space), and, consequently, the measurability must appear in the definitions. This will be highlighted in the next subsection. In addition, it is also worth mentioning that not all stochastic differential equation generate a random dynamical system (see [Arnold, 1998] for a more detailed exposition on this point). Specifically, in the case of stochastic partial differential equations, it is only known when the noise possesses a very particular form (e.g. either additive or linear multiplicative).

\subsection{Existence of pullback and random attractors for multi-valued non- autonomous and random dynamical systems}

Although we could have developed our theory for the existence of pullback attractors for processes or cocycles directly in the multi-valued case, we have preferred to first introduce and establish the theory in the single-valued framework because, in this way, it may be clearer for a general audience. Now, we will establish the theory in the more general set-up of multi-valued dynamical systems generalizing the theory in Section 4 for non-autonomous/random dynamical systems (see [Caraballo et al., 2008] for more details).

A pair $(\Omega, \theta)$, where $\Omega$ is a set and $\theta=\left(\theta_{t}\right)_{t \in \mathbb{R}}$ is a flow on $\Omega$, i.e., with $\theta: \mathbb{R} \times \Omega \rightarrow \Omega$ satisfying

$\theta_{0}=\mathrm{id}_{\Omega}, \quad \theta_{t+\tau}=\theta_{t} \circ \theta_{\tau}=: \theta_{t} \theta_{\tau} \quad$ for $t, \tau \in \mathbb{R}$

is called a non-autonomous perturbation. As an example which describes typical non-autonomous perturbations we consider $\Omega=\mathbb{R}$ and $\theta_{t} \tau=t+\tau$ for $\tau=\omega \in \Omega, t \in \mathbb{R}$.

Let $\mathcal{P}:=(\Omega, \mathcal{F}, \mathbb{P})$ be a probability space. On this probability space we consider a measurable non-autonomous flow $\theta$ :

$$
\theta:(\mathbb{R} \times \Omega, \mathcal{B}(\mathbb{R}) \otimes \mathcal{F}) \rightarrow(\Omega, \mathcal{F}) .
$$

In addition, $\mathbb{P}$ is supposed to be ergodic with respect to $\theta$, which means that every $\theta_{t}$-invariant set has measure zero or one, $t \in \mathbb{R}$. Hence $\mathbb{P}$ is invariant with respect to $\theta_{t}$. The quadruple $(\Omega, \mathcal{F}, \mathbb{P}, \theta)$ which is the model for a noise is called a metric dynamical system.

If we replace in the definition of a metric dynamical system the probability space $\mathcal{P}$ by its completion $\mathcal{P}^{c}:=(\Omega, \overline{\mathcal{F}}, \overline{\mathbb{P}})$ the above measurability property is not true in general, see Appendix A [Arnold, 1998; Appendix A]. But for fixed $t \in \mathbb{R}$ we have that the mapping

$$
\theta_{t}:(\Omega, \overline{\mathcal{F}}) \rightarrow(\Omega, \overline{\mathcal{F}})
$$

is measurable.

From now on, let $X=\left(X, d_{X}\right)$ be a Polish space.

Let $D: \omega \rightarrow D(\omega) \in 2^{X}$ be a multi-valued mapping. The set of multi-functions $D: \omega \rightarrow$ $D(\omega) \in 2^{X}$ with closed and non-empty images is denoted by $C(X)$. Let also denote by $P_{f}(X)$ the set of all non-empty closed subsets of the space $X$. Thus, it is equivalent to write that $D$ is in $C(X)$, or $D: \Omega \rightarrow P_{f}(X)$.

Let $D: \omega \rightarrow D(\omega)$ be a multi-valued mapping in $X$ over $\mathcal{P}$. Such a mapping is called a random set if

$$
\omega \rightarrow \inf _{y \in D(\omega)} d_{X}(x, y)
$$

is a random variable for every $x \in X$. It is well known that a mapping is a random set if and only if for every open set $O$ in $X$ the inverse image $\{\omega: D(\omega) \cap O \neq \emptyset\}$ is measurable, i.e., it belongs 
to $\mathcal{F}$ (see $[\mathrm{Hu} \&$ Papageorgiou, 1997; Proposition 2.1.4]).

Clearly, all this is also valid if we replace $\mathcal{P}$ by $\mathcal{P}^{c}$ and $\mathcal{F}$ by $\overline{\mathcal{F}}$. If we do not specify which probability space we are using $\left(\mathcal{P}\right.$ or $\left.\mathcal{P}^{c}\right)$, it will mean that the result is valid for both cases.

It is also evident that if $D$ is a random set with respect to $\mathcal{P}$, then it is also random with respect to $\mathcal{P}^{c}$.

We now introduce multi-valued nonautonomous and random dynamical systems.

Definition 5.42. A multi-valued map $U: \mathbb{R}^{+} \times$ $\Omega \times X \rightarrow P_{f}(X)$ is called a multi-valued nonautonomous dynamical system (MNDS) if

i) $U(0, \omega, \cdot)=\operatorname{id}_{X}$,

ii) $U(t+\tau, \omega, x) \subset U\left(t, \theta_{\tau} \omega, U(\tau, \omega, x)\right)$ for all $t, \tau \in \mathbb{R}^{+}, x \in X, \omega \in \Omega$.

It is called a strict MNDS if

iii) $U(t+\tau, \omega, x)=U\left(t, \theta_{\tau} \omega, U(\tau, \omega, x)\right)$ for all $t, \tau \in \mathbb{R}^{+}, x \in X, \omega \in \Omega$.

An MNDS is called a multi-valued random dynamical system (MRDS) if the multi-valued mapping

$$
(t, \omega, x) \rightarrow U(t, \omega, x)
$$

is $\mathcal{B}\left(\mathbb{R}^{+}\right) \otimes \mathcal{F} \otimes \mathcal{B}(X)$ measurable, i.e. $\{(t, \omega, x)$ : $U(t, \omega, x) \cap O \neq \emptyset\} \in \mathcal{B}\left(\mathbb{R}^{+}\right) \otimes \mathcal{F} \otimes \mathcal{B}(X)$ for every open set $O$ of the topological space $X$.

For the above composition of multi-valued mappings we use that for any non-empty set $V \subset$ $X, U(t, \omega, V)$ is defined by

$$
U(t, \omega, V)=\bigcup_{x_{0} \in V} U\left(t, \omega, x_{0}\right) .
$$

We also note that the above measurability hypothesis is not standard at least for single-valued random dynamical system. However, for MRDS it is more difficult to derive measurability than for single valued systems.

We now introduce some topological properties of the MNDS $U$.

Definition 5.43. $U(t, \omega, \cdot)$ is called upper semicontinuous at $x_{0}$ if for every neighborhood $\mathcal{U}$ of the set $U\left(t, \omega, x_{0}\right)$ there exists $\delta>0$ such that if $d_{X}\left(x_{0}, y\right)<\delta$ then

$$
U(t, \omega, y) \in \mathcal{U}
$$

$U(t, \omega, \cdot)$ is called upper semi-continuous if it is upper semi-continuous at every $x_{0}$ in $X$.

Remark 5.44. (i) Note that if a mapping $U(t, \omega, \cdot)$ is upper semi-continuous at $x_{0}$, then for all $\varepsilon>0$ there exists $\delta(\varepsilon)>0$ such that

$$
\operatorname{dist}_{X}\left(U(t, \omega, y), U\left(t, \omega, x_{0}\right)\right) \leq \varepsilon,
$$

for any $y$ satisfying $d_{X}\left(y, x_{0}\right) \leq \delta(\varepsilon)$.

(ii) The converse is true when $U\left(t, \omega, x_{0}\right)$ is compact, see [Aubin \& Cellina, 1984].

It is not difficult to extend Definition 5.43 if we consider the upper-semi-continuity with respect to all variables assuming that $\Omega$ is a Polish space.

We now formulate a general condition ensuring that an MNDS defines an MRDS. We need the particular assumption that $\Omega$ is a Polish space and $\mathcal{F}$ the associated Borel- $\sigma$-algebra.

Lemma 5.45. Let $\Omega$ be a Polish space and let $\mathcal{F}$ be the Borel- $\sigma$-algebra. Suppose that $(t, \omega, x) \mapsto$ $U(t, \omega, x)$ is upper semi-continuous. Then this mapping is measurable in the sense of Definition 5.42.

Proof. Thanks to Proposition 1.2.5 in [Hu \& Papageorgiou, 1997], we have that for each closed subset $C \subset X$, the set

$$
U^{-1}(C)=\{(t, \omega, x): U(t, \omega, x) \cap C \neq \emptyset\}
$$

is closed, and thus is a Borel set in $\mathcal{B}\left(\mathbb{R}^{+}\right) \otimes \mathcal{F} \otimes$ $\mathcal{B}(X)=\mathcal{B}\left(\mathbb{R}^{+} \times \Omega \times X\right)$. This implies that the inverse of a closed set is measurable and then, by Theorem 2.2.4 in [Hu \& Papageorgiou, 1997], the map is measurable.

\section{8(A). Non-autonomous and random attrac- tors for MNDS}

In this section we generalize the concept of pullback and random attractors to the case of an MNDS and prove a general result for the existence and uniqueness of attractors. 
As a preparation we need the following definitions.

A multi-valued mapping $D$ is said to be negatively, strictly, or positively invariant for the MNDS $U$ if

$$
\begin{aligned}
D\left(\theta_{t} \omega\right) & \subset \\
& \supset
\end{aligned}(t, \omega, D(\omega)) \quad \text { for } \omega \in \Omega, t \in \mathbb{R}^{+} .
$$

Let $\mathcal{D}$ be the family of multi-valued mappings with values in $C(X)$. We say that a family $K \in \mathcal{D}$ is pullback $\mathcal{D}$-attracting if for every $D \in \mathcal{D}$ and all $\omega \in \Omega$

$$
\lim _{t \rightarrow+\infty} \operatorname{dist}_{X}\left(U\left(t, \theta_{-t} \omega, D\left(\theta_{-t} \omega\right)\right), K(\omega)\right)=0 .
$$

$B \in \mathcal{D}$ is said to be pullback $\mathcal{D}$-absorbing if for every $D \in \mathcal{D}$ there exists $T=T(\omega, D)>0$ such that

$$
U\left(t, \theta_{-t} \omega, D\left(\theta_{-t} \omega\right)\right) \subset B(\omega), \text { for all } t \geq T .
$$

The following definition provides the main objective of this article. We have to introduce a particular set system (see [Schmalfuß, 2000]): let $\mathcal{D}$ be a set of multi-valued mappings in $C(X)$ satisfying the inclusion closed property: suppose that $D \in \mathcal{D}$ and let $D^{\prime}$ be a multi-valued mapping in $C(X)$ such that $D^{\prime}(\omega) \subset D(\omega)$ for $\omega \in \Omega$, then $D^{\prime} \in \mathcal{D}$.

Definition 5.46. A family $A \in \mathcal{D}$ is said to be a global pullback $\mathcal{D}$-attractor for the MNDS $U$ if it satisfies:

1. $A(\omega)$ is compact for any $\omega \in \Omega$;

2. $A$ is pullback $\mathcal{D}$-attracting;

3. $A$ is negatively invariant.

$A$ is said to be a strict global pullback $\mathcal{D}$ attractor if the invariance property in the third item is strict.

A natural modification of this definition for MRDS is

Definition 5.47. Suppose $U$ is an MRDS and suppose that the properties of Definition 5.46 are satisfied. In addition, we suppose that $A$ is a random set, with respect to $\mathcal{P}^{c}$. Then $A$ is called a random global pullback $\mathcal{D}$-attractor.
Remark 5.48. In contrast to the theory of random attractors for single valued random dynamical systems we have weaker assumptions on the measurability of $A$. Of course, it is desirable to obtain that $A$ is a random set with respect to $\mathcal{P}$, but usually we need stronger assumptions in the applications to obtain this property.

A consequence of the pullback convergence and invariance of $\mathbb{P}$ is that it reflexes the forward convergence to the attractor

$$
\mathbb{P}-\lim _{t \rightarrow+\infty} \operatorname{dist}_{X}\left(U(t, \omega, D(\omega)), A\left(\theta_{t} \omega\right)\right)=0
$$

for all sets $D$ such that $\omega \rightarrow U(t, \omega, D(\omega))$ is measurable for $t \geq 0$. Indeed, we have to replace in the formula for the pullback convergence $\omega$ by $\theta_{t} \omega$. However, this is only true in the weaker convergence in probability. There exist counterexamples which show that in general the forward convergence does not hold almost surely (see [Arnold, 1998; page 488]).

The main tool to prove the existence of an attractor is the pullback-omega-limit set for the MNDS $U$. For some multi-valued mappings $D$ we define a pullback-omega-limit set as an $\omega$ dependent set $\Lambda(D, \omega)$ given by

$$
\Lambda(D, \omega)=\bigcap_{s \geq 0} \overline{\bigcup_{t \geq s} U\left(t, \theta_{-t} \omega, D\left(\theta_{-t} \omega\right)\right)} .
$$

This set is obviously closed, but in general it can be empty. It is not difficult to prove that $y \in \Lambda(D, \omega)$ if and only if there exist $t_{n} \rightarrow$ $+\infty$ and $y_{n} \in U\left(t_{n}, \theta_{-t_{n}} \omega, D\left(\theta_{-t_{n}} \omega\right)\right)$ such that $\lim _{n \rightarrow+\infty} y_{n}=y$.

We then have the following lemma, which is a generalization of Theorem 6 and Lemma 8 in [Caraballo et al., 2003b] to the case in which we consider the family $\mathcal{D}$ instead of the bounded sets of $X$.

Lemma 5.49. Suppose that the $\operatorname{MNDSU}(t, \omega, \cdot)$ is upper semi-continuous for $t \geq 0$ and $\omega \in \Omega$. Let $B$ be a multi-valued mapping such that the MNDS is asymptotically compact with respect to $B$, i.e., for every sequence $t_{n} \rightarrow+\infty, \omega \in \Omega$ every sequence $y_{n} \in U\left(t_{n}, \theta_{-t_{n}} \omega, B\left(\theta_{-t_{n}} \omega\right)\right)$ is pre-compact.

Then for $\omega \in \Omega$ the pullback-omega-limit set $\Lambda(B, \omega)$ is non-empty, compact, and

$$
\lim _{t \rightarrow+\infty} \operatorname{dist}_{X}\left(U\left(t, \theta_{-t} \omega, B\left(\theta_{-t} \omega\right)\right), \Lambda(B, \omega)\right)=0,
$$


$\Lambda\left(B, \theta_{t} \omega\right) \subset U(t, \omega, \Lambda(B, \omega))$, for all $t \geq 0$.

The main theorem of this section is

Theorem 5.50. Assume the hypotheses in Lemma 5.49. In addition, suppose that $B \in \mathcal{D}$ is pullback $\mathcal{D}$-absorbing. Then, the set $A$ given by

$$
A(\omega):=\Lambda(B, \omega)
$$

is a pullback $\mathcal{D}$-attractor. Furthermore, $A$ is the unique element from $\mathcal{D}$ with these properties.

In addition, if $U$ is a strict MNDS then $A$ is strictly invariant.

With respect to the applicability of the measurability of Definition 5.47 in the applications, we suppose for the next lemma a complete probability space $\mathcal{P}^{c}$. However, the result is also valid for the space $\mathcal{P}$.

Lemma 5.51. Assume that $U$ is a MRDS. Under the assumptions in Theorem 5.50, let $\omega \rightarrow$ $U(t, \omega, B(\omega))$ be a random set for $t \geq 0$. Assume also that $U(t, \omega, B(\omega))$ is closed for all $t \geq 0$ and $\omega \in \Omega$. Then the set $A$ introduced in Theorem 5.50 is measurable, and therefore, it is a random global pullback $\mathcal{D}$-attractor.

\section{8(B). Applications and examples}

The autonomous examples considered in Section 4 can all be modified to give examples of non-autonomous multi-valued systems. Some additional specific references are [Caraballo et al., 2006, Kloeden \& Marín-Rubio, 2003,

Kloeden \& Schmalfuß, 1998,

Kloeden \& Valero, 2005], which also consider weak attractors, i.e., for which the invariance and attraction properties hold for at least one (rather than all) trajectories emanating from each point.

\section{Acknowledgements}

We are very grateful to our late Professor Valery S. Melnik for his encouragement, his constant support and his friendship. We will keep him forever in our hearts.

This paper has been partially supported by Ministerio de Ciencia e Innovación (Spain)
Projects MTM2005-03860, MTM2005-06098-C0201, MTM2008-00088 and MTM2009-11820, by Fundación Séneca (Comunidad Autóma de la regió de Murcia) Project $08667 / \mathrm{PI} / 08$, by JCCM Projects PAI06-0114 and PBC05-011-3 and the Consejería de Innovación, Ciencia y Empresa (Junta de Andalucía), Proyecto de Excelencia P07-FQM-02468.

\section{References}

Abraham, C., Biau, G. \& Cadre,B., [2004] "On Lyapunov exponents and sensitivity," J. Math. Anal. Appl. 290, 395-404.

Adler, R.L., Konheim, A.G. \& McAndrew, M.H. [1965] "Topological entropy," Trans.Amer.Math.Soc., 114, 309-319.

Alsedà, L., Llibre, J. \& Misiurewicz, M. [2000] Combinatorial dynamics and entropy in dimension one, second edition, (World Scientific, Singapore).

AlSharawi, Z., Angelos, J., Elaydi, S. \& Rakesh, L. [2006], "An extension of Sharkovsky's theorem to periodic difference equations," J.Math. Anal. Appl., 316, 128-141.

Amigó, J.M., Catto, I, Giménez, A. \& Valero, J. [2009] "Attractors for a non-linear parabolic equation modelling suspension flows," Discrete Contin. Dyn. Syst. Series B 11, 205-231.

Angelis, V. de. [2004], "Notes on the nonautonomous Lyness equation," preprint (2004)

Arnold, L. [1998] Random Dynamical Systems (Springer-Verlag, Berlin).

Arrieta, J., Carvalho, A.N. \& Hale, J.K. [1992] "A damped hyperbolic equation with critical exponent," Comm. Partial Differential Equations 17, 841-866.

Arrieta, J.M., Rodríguez-Bernal, A. \& Valero, J. [2006] "Dynamics of a reaction-diffusion equation with a discontinuous nonlinearity," Internat. J. Bifur. Chaos 16, 2695-2984.

Aubin, J.P. \& Celina, A. [1984] Differential Inclusions (Springer-Verlag, Berlin). 
Alves, J.F. [2009], "What we need to find out the periods of a periodic difference equation," Instituto Superior Técnico de Lisboa, Portugal, preprint.

Babin, A.V. [1995] "Attractor of the generalized semi-group generated by an elliptic equation in a cylindrical domain," Russian Acad. Sci. Izv. Math. 58, 207-223.

Babin, A.V. \& Vishik, M.I. [1985] "Maximal attractors of semi-groups corresponding to evolutionary differential equations," Math. Sbornik 126, 397-419.

Babin, A.V. \& Vishik, M.I. [1985] "Attracteurs maximaux dans les équations aux dérivées partielles," Nonlinear partial differential equations and their applications (Eds. H. Brezis \& J.L. Lions) (Research Notes in Math N.122, Pitman), 11-34.

Babin, A.V. \& Vishik, M.I. [1992] Attractors of Evolution Equations (Nord Holland, Amsterdam).

Balibrea, F., Cánovas, J. \& Jiménez López, V. [1999a] "Some results on entropy and sequence entropy," Internat. J. Bifur. Chaos 9, 17311742 .

Balibrea, F., Cánovas, J. \& Jiménez López, V. [1999b] "Commutativity and nonCommutativity of topological sequence entropy," Annales de L'Institut Joseph Fourier 495, 1693-1709.

Balibrea, F., Downarowicz, T., Hric,R., Snoha, L. \& Spitalský, V. [2009] "Almost totally disconnected minimal systems," Ergod. Th. E3 Dynam. Sys., 29, 737-766.

Balibrea, F., Hric,R., \& Snoha, L. [2003], Internat. J. Bifur. Chaos, 13 (7), 1721-1725.

Balibrea F., Esquembre F. \& Linero A. [1995] "Smooth triangular maps of type $2^{\infty}$ with positive topological entropy," Internat. J. Bifur. Chaos 88(1-2), 1319-1324.

Balibrea, F. \& García Guirao, J. [2005] "Continua with empty interior as $\omega$-limit sets," Appl. Gen. Topol. 6 (2), 195-205.
Balibrea, F.\& Jiménez López, V. [1999] "The measure of scrambled sets: a survey," Acta Univ. M Belli, Math no.7, 3-11.

Ball, J.M. [1978] "On the asymptotic behavior of generalized processes with applications to nonlinear evolution equations," J. Differential Equations 27, 224-265.

Ball, J.M. [1997] "Continuity properties and global attractors of generalized semi-flows and the Navier-Stokes equations," J. Nonlinear Science 7, 475-502.

Ball., J.M. [2000] "Continuity properties and global attractors of generalized semi-flows and the Navier-Stokes equations," in Mechanics: from theory to computation, pp.447-474 (Springer, New York).

Ball., J.M. [2004] "Global attractors for damped semi-linear wave equations," Discrete Contin. Dyn. Syst. 10, 31-52 .

Barbashin, E.A. [1948] "On the theoy of generalized dynamical systems," Moskov. Gos. Ped. Inst. Uchen. Zap. 135, Mat. 2, 110-133.

Barbashin, E.A. \& Alimov, Ju. I. [1961] "On the theory of dynamical systems with multi-valued and discontinuous characteristics," Dokl. Akad Nauk SSR 140, 1111-1113.

Barrio A., "Sensibilidad respecto a las condiciones iniciales para aplicaciones del intervalo", Doctoral Memoir, Universidad de Murcia (Spain), (2007)

Bates, P.W. \& Zheng, S. [1992] "Inertial manifolds and inertial sets for the phase-field equations," J. Dynamics Differential Equations 4, 375-398.

Bessaih, H. \& Flandoli, F. [2000] "Weak attractor for a dissipative Euler equation," J. Dynamics Differential Equations 12, 713-732.

Birnir, B. \& Svanstedt, N. [2004] "Existence and strong attractors for the Rayleigh-Bénard problem with a large aspect radio," Discrete Continuous Dynamical Systems 10, 53-74.

Blanchard F., Huang W. and Snoha L.[2008] "Topological size of scrambled sets," Colloqium Mathematicum 10 (2), 293-361. 
Block, L. \& Coppel, W. [1992] Dynamics in one dimension (Lecture Notes in Math. 1513 Springer-Verlag, Berlin).

Bowen R., [1970] "Topological entropy and axiom A, " Glob. Anal. Proc. Symp. Pure Math. 14, $23-41$.

Bondarevski, V.G. [1997] "Energetic systems and global attractors for the 3D Navier-Stokes equations," Nonlinear Anal. 30, 799-810.

Bridgland, T.F. [1969] "Contributions to the theory of generalized differential equations. I," Math. Systems Theory 3, 17-50.

Bridgland, T.F. [1969] "Contributions to the theory of generalized differential equations. II," Math. Systems Theory 3, 156-165.

Brochet, D., Hilhorst, D. \& Chen, X. [1993] "Finite dimensional exponential attractor for the phase field model," Appl. Anal. 49 197-212.

Bronstein, I.U. [1963] "On dynamical systems without uniqueness assemi-groups of nonsinglevalued mappings of a topological space," Izv. Akad. Nauk Moldav. SSR 1, 3-17. English translation in Amer. Math. Soc. Transl. 97 (1970), 205-225.

Brunovsky, P. \& Fiedler, B. [1989] "Connecting orbits in scalar reaction diffusion equations II. The complete solution," J. Differential Equations 81, 186-135.

Budak, B.M. [1952] "The concept of motion in a generalized dynamical system," Moskov. Gos. Ped. Inst. Uchen. Zap. 155, Mat. 5, 174-194. English translation in Amer. Math. Soc. Transl. 97 (1970), 181-204.

Budyko, M. I. [1969] "The efects of solar radiation variations on the climate of the Earth," Tellus 21, 611-619.

Bushaw, D. [1963] "Dynamical polysystems and optimization," Contributions to Differential Equations 2, 351-365.

Camouzis, E. \& Ladas, G. [2007] Dynamics of third-order rational difference equations with open problems and conjectures (Chapman \& Hall/CRC Press, Volume 5).
Cánovas, J. [2010] "Analyzing when dynamic Parrondo's paradox is not possible," Internat. J. Bifur. Chaos, this issue.

Cánovas, J. \& Linero, A. [2006] "Periodic structure of alternating continuous interval maps," J. Difference Equ. Appl. 12(2), 847-858.

Cánovas, J., Linero, A. \& Peralta-Salas, D. [2006] "Dynamic Parrondo's paradox," Physica D., 218, 177-184.

Caraballo, T., Kloeden, P.E. \& Marín-Rubio, P. [2004] "Global and pullback attractors of setvalued skew product flows", Annali Matem. Pura Appl. 185, S23-S45.

Caraballo, T., Kloeden, P.E. \& Real, J. [2004] "Pullback and forward attractors for a damped wave equation with delays," Stochastics $\&$ $D y$ namics 4 (3), 405-423.

Caraballo, T., Marin-Rubio, P. \& Robinson, J. C. [2003] "A comparison between two theories for multi-valued semi-flows and their asymptotic behavior," Set Valued Anal. 11, 297-322.

Caraballo, T., Marín-Rubio, P. \& J.Valero [2005] "Autonomous and non-autonomous attractors for differential equations with delays," J. Differential Equations 208, 9-41.

Caraballo, T., Marín-Rubio, P. \& J.Valero [2007] "Attractors for differential equations with unbounded delays," J. Differential Equations 239, 311-342.

Caraballo, T. \& Real, J. [2004] "Attractors for 2DNavier-Stokes models with delays," J. Differential Equations 205, 270-296.

Caraballo, T., Garrido-Atienza, M.J., Schmalfuß, B. \& Valero, J. [2008] "Non-autonomous and random attractors for delay random semi-linear equations witout uniqueness," Discrete Contin. Dyn. Syst. Series A 21 (2), 415-443.

Caraballo, T., Langa, J. A., Melnik, V. S. \& Valero, J. [2003] "Pullback attractors of nonautonomous and stochastic multi-valued dynamical systems" Set Valued Anal., 1 (2), 153-201. 
Carvalho, A.N. \& Gentile, C.B. [2003] "Asymptotic behavior of non-linear parabolic equations with monotone principal part," J. Math. Anal. Appl. 280, 252-272.

Carvalho, A.N., Langa, J.A., \& Robinson, J.C., [2010] Attractors of Infinite Dimensional nonautonomous Dynamical Systems (to appear).

Chacón, R. [2001] "Maintenance and suppression of chaos by weak harmonic perturbations: a unified view," Phys. Rev. Lett. 86 (9), 1737-1740.

Cheban, D. [2004] Global Attractors of Nonautonomous Dissipative Dynamical Systems (World Scientific, New York).

Cheban, D. \& Fakeeh, D. [1994] Global Attractors of Dynamical Systems without Uniqueness (Sigma, Kishinev).

Cheban, D.N. \& Fakhih, D.S. [1994] The Global Attractors of Dynamical Systems without Uniqueness (Sigma, Kishinev).

Cheban, FD., P.E. Kloeden, P.E., \& Schmalfuß, B. [2002], "The Relationship between Pullback, Forward and Global Attractors of Nonautonomous Dynamical Systems", Nonlinear Dynamics and Systems Theory 2 (2), 125-144.

Cheban, D. \& Mammana, C. [2006] "Compact global attractors of discrete inclusions," Nonlinear Anal. 65, 1669-1687.

Chepyzhov, V.V., Gatti, S., Grasselli, M., Miranville, A. \& Patta, V. [2006] "Trajectory and global attractors for evolutions equations with memory," Applied Mathematics Letters 19, 8796.

Chepyzhov, V.V. \& Vishik, M.I. [1996] "Trajectory attractors for reaction-diffusion systems," Topological Methods in Nonlinear Analysis 7, 49-76.

Chepyzhov, V.V. \& Vishik, M.I. [1997] "Evolution equations and their trajectory attractors," $J$. Math. Pure Appl. 76, 913-964.

Chepyzhov, V.V. \& Vishik, M.I. [2002a] "Trajectory and global attractors for 3D Navier-Stokes system," Mat. Zametki 71, 194-213.
Chepyzhov, V.V. \& Vishik, M.I. [2002b] Attractors for equations of mathematical physics (American Mathematical Society, Providence, Rhode Island).

Cheskidov, A. [2006] "Global attractors of evolutionary systems," Archiv: math.DS/060935\%.

Cheskidov, A. \& Foias, C. [2006] "On global attractors of the 3D Navier-Stokes equations," $J$. Differential Equations 231, 714-754.

Constantin, P. [2007] "On the Euler equations of incompressible fluids," Bull. Amer. Math. Soc. 44, 603-621.

Cutland, N.J. [2005] "Global attractors for small samples and germs of 3D Navier-Stokes equations," Nonlinear Anal. 62, 265-281.

Crauel, H. \& Flandoli, F. [1994] "Attractors for random dynamical systems," Probability Theory and Related Fields 100 (3), 365-393.

Crauel, H., Debussche, A. \& Flandoli, F. [1995] "Random attractors," J. Dynamics Differential Equations 9 (2), 307-341.

Dafermos, C.M. [1971] "An invariance principle for compact processes", J. Differential Equations 9, 239-252.

Díaz, G., \& Díaz, J. I. [2002] "On a stochastic parabolic PDE arising in Climatology," Rev. R. Acad. Cien. Serie A Mat. 96, 123-128.

Díaz, J.I., Hernández \& J., Tello, L. [1997] "On the multiplicity of equilibrium solutions to a nonlinear diffusion equation on a manifold arising in Climatology," J. Math. Anal. Appl. 216, 593613.

Díaz, J.I., Hernández \& J., Tello, L. [2002] "Some results about multiplicity and bifurcation of stationary solutions of a reaction diffusion climatological model," Rev. R. Acad. Cien. Serie A. Mat 96, 357-366.

Dinaburg, E.I., [1970] "The relation between topological and metric entropy," Soviet Math. 11, 1316. 
Efendiev, M. \& Zelik, S. [2001] "The attractor for a nonlinear reaction-diffusion system in an unbounded domain," Comm. Pure Appl. Math. 54, 625-88.

Efremova, L. \& Makhrova, E. [2003] "On the center of continuous maps on dendrites," J. Diff. Equa. Appl. 9 (3/4), 381-392.

Elmounir O. \& Simonolar F. [2000] "Abstracteurs compacts pur des problèmes d'evolution sans unicité," Annales de la Faculté des Sciences de Toulouse IX (Série 6), 631-654.

Feireisl E. \& Norbury J. [1991] "Some existence and nonuniqueness theorems for solutions of parabolic equations with discontinuous nonlinearities," Proc. Roy. Soc. Edinburgh 119A, 117.

Feireisl, E. [2000] "Global attractors for the NavierStokes equations of three-dimensional comprenssible flow," C.R.Acad.Sci. Paris, Série I 331, 35-39.

Fiedler, B. \& Rocha, C. [1996] "Heteroclinic orbits of semi-linear parabolic equations," J. Differential Equations 125, 239-281.

Flandoli, F. \& Schmalfuß, B. [1996] "Random attractors for the 3D stochastic NavierStokes equation with multiplicative white noise," Stochastics and Stochastics Reports 59, 21-45.

Flandoli, F. \& Schmalfuß, B. [1999] "Weak solutions and attractors for three-dimensional Navier-Stokes equations with nonregular force," J. Dynamics Differential Equations 11, 355-398.

Foias, C., Manley, O.P., Rosa R. \& Temam, R. [2001] Navier-Stokes Equations and Turbulence (Cambridge University Press, Cambridge).

Ghidaglia, J.M. [1994] "A note on the strong convergence towards attractors of damped forced KdV equations," J. Differential Equations 110, 356-359.

Ghidaglia, J.M. \& Temam, R. [1985] "Properties of the attractors associated to damped hyperbolic equations," Comptes Rendus Acad. Sci. Paris 300, 185-188.
Ghidaglia, J.M. \& Temam, R. [1987] "Attractors for damped nonlinear hyperbolic equations," $J$. Math. Pures Appl. 66, 273-319.

Hale, J.K. [1977] Introduction to Functional Differential Equations (Springer-Verlag, New York).

Hale, J.K. [1985] "Asymptotic behavior and dynamics in infinite dimensions," in Nonlinear differential equations (Ed. J.K.Hale \& $P$. Martinez-Amores), pp.1-42 (Research Notes in Math. N.132, Pitman).

Hale, J.K. [1988] Asymptotic Behavior of Dissipative Systems (Mathematical Surveys and Monographs, Vol.25, A.M.S., Providence).

Hale, J.K. \& Lasalle, J.P. [1972] "Theory of a general class of dissipative processes," J. Math. Anal. Appl. 39, 177-191.

Haraux, A. [1985] "Two remarks on hyperbolic dissipative problems," Nonlinear partial differential equations and their applications (Ed. H. Brezis \& J.L. Lions), pp. 161-179 (Research Notes in Math N.122, Pitman).

Harmer, G.P. \& Abbott, D. [1999] "Losing strategies can win by Parrondo's paradox," Nature 402, 864 .

Harmer, G.P. \& Abbott, D. [1999] "Parrondo's paradox," Stat.Sci. 14, 206-213.

Henry, D. [1985] "Some infinite-dimensional MorseSmale systems defined by parabolic partial differential equations," J. Differential Equations 59, 165-205.

Hetzer, G. "The shift-semi-flow of a multi-valued equation from climate modeling," Nonlinear Anal. 47, 2905-2916.

Hetzer, G. \& Tello, L. [2002] "On a reactiondiffusion system arising in climatology," $D y$ namic Systems and Applications 11, 381-402.

Horban, N.V. \& Stanzhyts'kyi, O.M. [2008] "Global attractor for the autonomous wave equation in $\mathbb{R}^{n}$ with continuous nonlinearity," Ukraïn. Mat. Zh. 60, 299-309.

Hu, S. \& Papageorgiou, N. S. [1997] Handbook of multi-valued analysis. Vol. I. Applicatons (Kluwer Academic Publishers, Dordrecht). 
Iovane, G. \& Kapustyan, A.V. [2005] "Global attractor for impulsive reaction-diffusion equations", Nonlinear Oscillations 8, 318-328.

Iovane, G. \& Kapustyan, O.V. [2006] "Global attractors for non-autonomous wave equation without uniqueness of solution," Syst. Research and Inf. Techn. 2, 107-120.

Jiménez-Casas, A. \& Rodríguez-Bernal, A. [2002] "Asymptotic behavior for a phase-field model in higher order Sobolev spaces," Rev. Mat. Complut. 15, 213-248.

Kalantarov, V.K. [1991] "On minimal global attractor of the system of phase-field equations," Zap. Nauch. Sem. LOMI 188, 70-87.

Kapustyan O.V. [1999] "Attractor of semi-flow, generated by phase-field equations system without uniqueness of solution," Ukraïn. Mat. Zh. 7, 1006-1009.

Kapustyan, O.V. [2002] "Global attractors of a nonautonomous reaction-diffusion equation," Diff. Uravnenya 38, 1378-1381. English translation in Differential Equations 38 (2002), 1467-1471.

Kapustyan, O.V., Melnik, V.S. \& Valero, J. [2003] "Attractors of multi-valued dynamical processes generated by phase-field equations," Internat. $J$. Bifur. Chaos 13, 1969-1984.

Kapustyan, O.V., Melnik, V.S. \& Valero, J. [2007] "A weak attractor ans properties of solutions for the three-dimensional Bénard problem, "Discrete Contin. Dyn.. Systems 18 (2007), 449-481.

Kapustyan, O.V., Mel'nik, V.S., Valero, J. \& Yasinsky, V.V. [2008] Global atractors of multi-valued dynamical systems and evolution equations without uniqueness (Naukova Dumka, Kyiv).

Kapustyan, O.V. \& Shkundin, D.V. [2003] "Global attractor of one nonlinear parabolic equation," Ukrain. Mat. Zh. 55, 446-455. English translation in Ukrain. Math. J. 55 (2003), 535-547.

Kapustyan, O.V. \& Valero, J. [2000] "Attractors of multi-valued semi-flows generated by differential inclusions and their approximations," Abstr. Appl. Anal. 5, 33-46.
Kapustyan, O.V. \& Valero, J. [2006] "On the connectedness and asymptotic behavior of solutions of reaction-diffusion systems," J. Math. Anal. Appl. 323, 614-633.

Kapustyan, O.V. \& Valero, J. [2007] "Weak and strong attractors for 3D Navier-Stokes system," J. Differential Equations 240, 249-278.

Kapustyan, O.V. \& Valero, J. [2009a] "On the Kneser property for the Ginzburg-Landau equation and the Lotka-Volterra system with diffusion," J. Math. Anal. Appl. 357, 254-272.

Kapustyan, O.V. \& Valero, J. [2009b] "Comparison between trajectory and global attractors for evolution systems without uniqueness of solutions," Internat. J. Bifur Chaos, this issue.

Kato, H. [1995] "A note on periodic points and recurrent points of maps of dendrites," Bull. Austral. Math. Soc. 51, 459-461.

Kato, H. [1998] "The depth of centres of maps on dendrites," J. Austral. Math. Soc. Ser. A 64, 4453.

Kenmochi, N. \& Yamazaki, N. [2001] "Global attractors for multi-valued flows associated with subdifferentials," Commun. Appl. Anal. 5, 403419.

Kloeden, P.E. [1974] "General control systems without backwards extension," in Differential Games and Control Theory (P.L.E. Roxin 8 R.Sternberg eds), pp. 49-58 (Marcel \& Dekker, New-York).

Kloeden, P.E. [1997] "Non-autonomous systems, cocycle attractors and variable time-step discretization", Numerical Algorithms 14, no. 1-3, 141-152.

Kloeden, P.E. [2000] "Pullback attractors in nonautonomous difference equations," J. Difference Equ. Appl. 6, 33-52.

Kloeden, P.E., and Li Zhong [2006] "Li-Yorke chaos in higher dimensions: a review", J. Difference Equ.Appl., 12 (3), 247-269.

Kloeden, P.E., \& Li, D. [2005] "Equi-attraction and continuous dependence of strong attractors of 
set-valued dynamical systems on parameters," Set-Valued Anal. 13, 405-416.

Kloeden, P.E., \& Marín-Rubio, P. [2003] "Weak pullback attractors of nonautonomous difference inclusions," J. Difference Equ. Appl., 9, 489-502.

Kloeden, P.E., \& Rasmussen, M. [2010], Nonautonomous Dyamical Systems (to appear).

Kloeden, P.E., \& Siegmund, S. [2005] "Bifurcations and continuous transitions of attractors in autonomous and non-autonomous systems", Internat. J. Bifur. Chaos 15 (3), 743-762.

Kloeden, P.E. \& Schmalfuß, B. [1996] "Lyapunov functions and attractors under variable timestep discretization," Discrete and Continuous Dynamical Systems 2 (2), 163-172.

Kloeden, P.E. \& Schmalfuß, [1998] "Asymptotic behavior of non-autonomous difference inclusions," Systems Control Lett. 33 (4), 275-280.

Kloeden, P.E. \& Valero, J. [2005] "Attractors of weakly asymptotically compact setvalued dynamical systems," Set-Valued Analysis 13, 381404.

Kloeden, P.E. \& Valero, J. [2007] "The weak connectedness of the attainability set of weak solutions of the three-dimensional Navier-Stokes equations," Proc. R. Soc. A 463, 1491-1508.

Kocan, Z., Kornecká, V., \& Málek, M. [2010] "On the center of $\omega$-limit points of continuous maps on dendrites," Topol. Appl. (to appear).

Kocic, V. \& Ladas, G. [1993] Global behavior of non-linear difference equations of higher order with applications (Kluwer Academic Publishers, Dordrecht.

Kolyada, S. \& Snoha, L. [1996] "Topological entropy of non-autonomous dynamical systems," Random Com. Dynamics, 4, 205-233.

Kolyada, S., Misiurewicz, M. \& Snoha, L. [1999] "Topological entropy of non-autonomous piecewise monotone dynamical systems on the interval," Fund. Math. 160, 161-181.
Kulenovic, M. \& Ladas, G. [2002] Dynamics of second order rational difference equations with open problems and conjectures (Chapman \& Hall, London).

Ladyzhenskaya, O.A. [1972] "Dynamical system, generated by Navier-Stokes equations," Zap. Nauch. Sem. LOMI 27, 91-115.

Ladyzhenskaya, O.A. [1982] "On finitedimensionality of bounded invariant sets for Navier-Stokes equations and other dissipative systems," Zapiski Nauchnih Seminarov LOMI 115, 137-155.

Ladyzhenskaya, O.A. [1987] "On finding minimal global attractors for the Navier-Stokes equations and other PDE," Uspekhi Mat. Nauk. 42, 25-60.

Ladyzhenskaya, O.A. [1990] "Some comments to my papers on the theory of attractors for abstract semi-groups," Zapiski Nauchnikh seminarov LOMI 182, 102-112. English translation in J. Soviet Math., 62 (1992), 1789-1794.

Ladyzhenskaya, O.A. [1991] Attractors for semigroups and Evolution Equations (Cambridge University Press, Cambridge).

Langa, J.A. \& Schmalfuß, B. [2004] "Finite dimensionality of attractors for non-autonomous dynamical systems given by partial differential equations," Stochastics and Dynamics 4 (3), 385-404.

Li, D., Wang, Y. \& Wang, S. [2008] "On the dynamics of non-autonomous general dynamical systems and differential inclusions," Set-Valued Anal. 16, 651-671.

Lions, J.L. [1969] Quelques méthodes de résolution des problèmes aux limites non linéaires (Gauthier-Villar, Paris).

Lu, K. \& Wang, B. [2001] "Global attractors for the Klein-Gordon-Schrödinger equation in unbounded domains," J. Differential Equations 170, 281-316.

Lukaszewicz, G. \& Sadowski, W. [2004] "Uniform attractor for 2D magneto-micropolar fluid flow in some unbounded domains," Z. Angew. Math. Phys. 55, 1-11. 
Mai, J.H. [2005] "Pointwise-recurrent graphs maps," Ergod.Th. \& Dynam.Sys. 25 (2), 629637.

Malek, J. \& Necas, J. [1996] "A finite-dimensional attractor for three dimensional flow of incompresible fluids," J. Differential Equations 127, 498-518.

Malek, J. \& Prazak, D. [2002] "Large time behavior via the method of l-trajectories," J. Differential Equations 181, 243-279.

Marion, M. [1987] "Attractors for reaction-diffusion equations: existence and estimate of their dimension," Appl. Anal. 25, 101-147.

Marotto, F.R. [1978] " Snap-back repellers imply chaos in $\mathbb{R}^{n}, "$ J. Math. Anal. Appl. 63, 109-223.

Melnik, V.S. [1994] "multi-valued dynamics of nonlinear infinite dimensional systems," Preprint of NAS of Ukraine, 94-17, Institute of Cybernetics, Kyiv.

Melnik, V.S. [1998] "Estimates of the fractal and hausdorff dimensions of sets invariant under multimappings," Math. Notes 63, 190-196.

Melnik, V.S. \& Valero, J. [1998] "On attractors of multi-valued semi-flows and differential inclusions," Set Valued Anal. 6, 83-111.

Melnik, V.S. \& Valero, J. [2008] "Addendum to "On attractors of multi-valued semi-flows and differential inclusions" [Set-Valued Anal., 6 (1998), 83-111]," Set Valued Anal. 16, 507-509.

Miller, R. K. [1965] "Almost periodic differential equations as dynamical systems with applications to the existence of almost periodic solutions", J. Differential Equations 1, 337-395.

Minkevic, M.I. [1948] "A theory of integral funnels for dynamical systems without uniqueness," Moskov. Gos. Ped. Inst. Uchen. Zap. 135, 134151. English translation in Amer. Math. Soc. Transl. 95 (1970), 11-34.

Misiurewicz, M. [1989], "Horseshoes for mappings of an interval," Bull. Acad. Pol. Sci., Sé. Sci. Math. 315, 167-169.
Misiurewicz; M. \& Szlenk, W.[1980], "Entropy of piecewise monotone mappings," Studia Math. 67 (1980), 45-63.

Moise, I., Rosa, R. \& Wang, X. [1998] "Attractors for non-compact semi-groups via energy equations," Nonlinearity 11, 1369-1393.

Morillas, F. \& Valero, J. [2005] "Attractors for reaction-diffusion equations in $R^{N}$ with continuous nonlinearity," Asymptot. Anal. 44, 111-130.

Morillas, F. \& Valero J. [2008] "Asymptotic compactness and attractors for phase-field equations in $\mathbb{R}^{3}, "$ Set-Valued Anal. 16, 861-897.

Morillas, F. \& Valero J. [2009] "A Peano's theorem and attractors for lattice dynamical systems," Internat J. Bifur. Chaos 19, 557-578.

Norman, D.E. [1999] "Chemically reacting fluid flows: weak solutions and global attractors," $J$. Differential Equations 152, 75-135.

Nadler, S.B. [1992] Continuum Theory: An Introduction, Monogrphs and Textbooks In Pure and Applied Math., Vol 158, Marcel Dekker, New York, Basel, Hong Kong, 1992.

Otani, M. [1977] "On existence of strong solutions for $\frac{d u}{d t}+\partial \psi^{1}(u(t))-\partial \psi^{2}(u(t)) \ni f(t)$, , J. Fac. Sci. Univ. Tokio. Sect. IA Math 24, 575-605.

Otani, M. [1984] "Nonmonotone perturbations for nonlinear parabolic equations associated with subdifferential operators, periodic problems problems," J. Differential Equations 54, 248273.

Papageorgiuou, N.S. \& Papalini, F. [1996] "On the structure of the solution set of evolution inclusions with time-dependent subdifferentials," Acta Math. Univ. Comenianae 65, 33-51.

Papaschinopolous, G. \& Schinas, C.J. [2008], "On a non-autonomous kth-order rational equation difference equation" J. Difference Equ. Appl. 14 (6), 645-655

Parrondo, J.M., Harmer, G.P. \& Abbott, D. [2000] "New paradoxical games based on brownian ratchets," Phys. Rev. Lett. 85, 5226-5229. 
Rosa, R. [1998] "The global attractor for the 2D Navier-Stokes flow on some unbounded domains," Nonlinear Anal. 32, 71-85.

Rosa, R. [2006] "Asymptotic regularity conditions for the strong convergence towards weak limit set and weak attractors of the 3D Navier-Stokes equations," J. Differential Equations 130, 196212.

Rossi, R., Segatti, A. \& Stefanelli, U. [2008] "Attractors for gradient flows of non convex functionals and appplications," Arch. Rational Mech. Anal. 187, 91-135.

Roxin, E. [1965] "Stability in general control systems," J. Differential Equations 1, 115-150.

Roxin, E. [1965] "On generalized dynamical systems defined by contingent equations," J. Differential Equations 1, 188-205.

Schimperna, G. [2007] "Global attractors for CahnHilliard equations with nonconstant mobility," Nonlinearity 20, 1987-2006.

Schmalfuß, B. [1992] "Backward cocycles and attractors of stochastic differential equations," in International seminar on Applied Mathematics - Nonlinear Dynamics: Attractor Approximation and Global behavior (Eds. V. Reitmann, T. Riedrich, and N. Koksch) (Technische Universität, Dresden), 185-192.

Schmalfuß, B. [2000] "Attractors for nonautonomous dynamical systems," in Proc. Equadiff 99, Berlin (Eds. B. Fiedler, K. Gröger and J. Sprekels) (World Scientific, 2000), 684-689.

Segatti, A. [2006] "Global attractor for a class of doubly nonlinear abstract evolution equations," Discrete Contin. Dyn. Systems 14, 801-820.

Segatti, A. [2007] "On the hyperbolic relaxation of the Cahn-Hilliard equation in 3D: approximation and long time behaviour," Math. Models Methods Appl. Sci. 17, 411-437.

Sell, G.R. [1971] Topological Dynamics and Ordinary Differential Equations, (Van Nostrand Reinhold Mathematical Studies, London).
Sell, G.R. [1996] "Global attractors for the threedimensional Navier-Stokes equations," J. Dynamics Differential Equations 8, 1-33.

Sell, G.R. \& You, Y. [1995] Dynamics of evolutionary equations (Springer, New-York).

Sharkovskii A.N. [1964] "Co-existence of the cycles of a continuous mappings of the line into itself" (in Ukrainian), Ukrain. Math. Zh. 16 (1), 64-71.

Sharkovskii, A.N. [1994] "Co-existence of the cycles of a continuous mappings of the line into itself" (in Ukrainian), Proceedings of the Conference "Thirty Years after Sharkovskii's theorem: New Perspectives" (Murcia, 1994), Internat. J. Bifur. Chaos 5 (1995), 1263-1273.

Shi, Y.M. \& "Discrete chaos in Banach spaces", Science in China Series A: Mathematics, 34, 595609 .

Shnirelman, A. [1997] "On the nonuniqueness of weak solution of the Euler equation," Comm. Pure Appl. Math. L, 261-1286.

Simsen, J. \& Gentile, C. [2008] "On attractors for multi-valued semi-groups defined by generalized semi-flows," Set-Valued Anal. 16, 105-124.

Spitalský, V. [2008] "Omega-limit sets in hereditarily locally connected continua," Topol. Appl. 155 (11), 1237-1255.

Smoller, J. [1983] Shock waves and reactiondiffusion equations (Springer, New-York).

Stuart, A.M. \& Humphries, A.R. [1996] Dynamical Systems and Numerical Analysis, (Cambridge University Press, Cambridge).

Szego, G.P. \& Treccani, G. [1969] Semi-grupi di transformazioni multivoche (Springer-Verlag, Berlin).

Temam, R. [1979] Navier-Stokes Equations (NorthHolland, Amsterdam).

Temam, R. [1988] Infinite-Dimensional Dynamical Systems in Mechanics and Physics SpringerVerlag, New York.

Terman D. [1983] "A free boundary problem arising from a bistable reaction-diffusion equation," Siam J. Math. Anal. 14, 1107-1129. 
Terman D. [1085] "A free boundary arising from a model for nerve conduction," J. Differential Equations 58, 345-363.

Tolstonogov, A.A. \& Umansky, Ya.I. [1992] "On solutions of evolution inclusions I," Siberian Math. J. 33, 500-511.

Tolstonogov, A.A. \& Umansky, Ya.I. [1992] "On solutions of evolution inclusions II," Siberian Math. J. 33, 693-702.

Valero, J. [2000] "Finite and infinite dimensional attractors of multi-valued reaction-diffusion equations", Acta Math. Hungary. 3, 239-258.

Valero, J. [2001] "Attractors of parabolic equations without uniqueness," J. Dynamics Differential Equations 13, 711-744.

Valero, J. [2005] "On the Kneser property for some parabolic problems", Topology Appl. 153, 975989.

Vishik, M.I. [1992] "Asymptotic behavior of solutions of evolutionary equations" (Cambridge Univ. Press, Cambridge).

Vorotnikov, D. \& Zvyagin, V. [2007] "Uniform attractors for non-autonomous motion equations of viscoelastic medium," J. Math. Anal. Appl. 325, 438-458.

Vrabie, I.I. [1997] Compactness Methods for Nonlinear Equations (Pitman Longman, London).

Wang, B. [1999] "Attractors for reaction-diffusion equations in unbounded domains," Physica D 128, 41-52.

Wang, Y. \& Zhou, Sh [2007] "Kernel sections and uniform attractors of multi-valued semiprocesses," J. Differential Equations 232, 573622.

Yamazaki, N. [2004] "Attractors of asymptotically periodic multi-valued dynamical systems governed by time-dependent subdifferentials," Elec. J. Differential Equations 107, 1-22.

Ye, X. [1993] "The center and the depth of the center of a tree map," Bull. Austral. Math. Soc. 48, 347-350. 